MPP-2004-50, LAPTH-1046/04, PM/04-13, ZU-TH-08/04, IFIC/04-21

\title{
Precise determination of the neutral Higgs boson masses in the MSSM
}

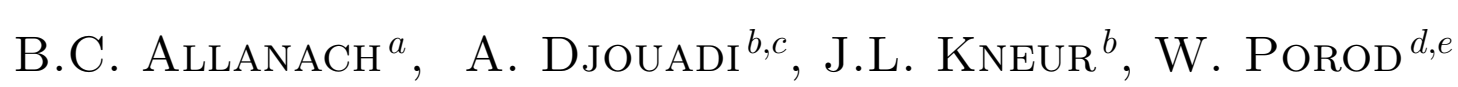 \\ and P. SLAVICH ${ }^{f}$ \\ ${ }^{a}$ LAPTH, 9 Chemin de Bellevue, F-74941 Annecy-le-vieux, France \\ ${ }^{b}$ LPMT, Université de Montpellier II, F-34095 Montpellier Cedex 5, France \\ ${ }^{c}$ LPTHE, Universités Paris 6877, 4 Place Jussieu, F-75252 Paris Cedex 05, France \\ ${ }^{d}$ IFIC - Instituto de Física Corpuscular, E-46071 Valencia, España \\ e Physik Institut, Universität Zurich, Winterthurerstrasse 190, CH-8057 Zurich, Switzerland \\ $f$ Max Planck Institut für Physik, Föhringer Ring 6, D-80805 München, Germany
}

\begin{abstract}
We present the implementation of the radiative corrections of the Higgs sector in three public computer codes for the evaluation of the particle spectrum in the Minimal Supersymmetric Standard Model, SoftSusy, SPheno and SuSpect. We incorporate the full one-loop corrections to the Higgs boson masses and the electroweak symmetry breaking conditions, as well as the two-loop corrections controlled by the strong gauge coupling and the Yukawa couplings of the third generation fermions. We include also the corrections controlled by the $\tau$ Yukawa coupling that we derived for completeness. The computation is consistently performed in the $\overline{\mathrm{DR}}$ renormalisation scheme. In a selected number of MSSM scenarios, we study the effect of these corrections and analyse the impact of some higher order effects. By considering the renormalisation scheme and scale dependence, and the effect of the approximation of zero external momentum in the two-loop corrections, we estimate the theoretical uncertainty on the lighter Higgs boson mass to be 3 to $5 \mathrm{GeV}$. The uncertainty on $M_{h}$ due to the experimental error in the measurement of the SM input parameters is approximately of the same size. Finally, we discuss the phenomenological consequences, using the latest value of the top quark mass. We find, in particular, that the most conservative upper bound on the lighter Higgs boson mass in the general MSSM is $M_{h} \lesssim 152 \mathrm{GeV}$ and that there is no lower bound on the parameter $\tan \beta$ from non-observation of the MSSM Higgs bosons at LEP2.
\end{abstract}

e-mail: benjamin.allanach@cern.ch, abdelhak.djouadi@cern.ch, kneur@lpm.univ-montp2.fr, porod@ific.uv.es, slavich@mppmu.mpg.de. 


\section{Introduction}

The Minimal Supersymmetric Standard Model (MSSM) [1] provides an attractive weak-scale extension of the Standard Model (SM). At the price of introducing a bosonic (fermionic) super-partner for every fermionic (bosonic) particle of the SM, plus an additional doublet of Higgs fields and their fermionic partners, it allows one to solve the hierarchy problem, leads to a consistent unification of the SM gauge couplings and provides a natural candidate for the dark matter in the universe. In the last two decades, a large effort has been devoted to the detailed theoretical study of the properties of the supersymmetric particles and the MSSM Higgs bosons, and to the experimental search for these particles, either directly in high-energy collider experiments or indirectly through high-precision measurements.

The task of providing a detailed phenomenological analysis of the masses and couplings of the supersymmetric particles and the MSSM Higgs bosons, and comparing it with the results or expectations of the present and future experiments, proves to be an extremely difficult one, due to the large number of new parameters ( $>100$ in the most general soft supersymmetry (SUSY) breaking Lagrangian and $>20$ when some phenomenological constraints are imposed; see Ref. 2] for a discussion). However, there are well motivated theoretical models where the soft SUSY-breaking parameters obey a number of universal boundary conditions at some very high scale, such as the Grand Unification Theory (GUT) scale $M_{\mathrm{GUT}}$, or the messenger scale $M_{\text {mess }}$, thus reducing the number of fundamental parameters to a handful. This is for instance the case of the models of gravity mediated (mSUGRA) [3], gauge mediated (GMSB) 44 or anomaly mediated (AMSB) [5] SUSY breaking. String-inspired models of soft SUSY-breaking have also been proposed [6]. In all such models, the soft SUSY-breaking parameters are given as input at the high-energy scale, then they are evolved by means of the MSSM Renormalisation Group Equations (RGE) down to a low-energy scale, $M_{\text {EWSB }}$, where the conditions of electroweak symmetry breaking (EWSB) are imposed and the spectrum of superparticle masses and couplings is computed.

Several computer codes (that in what follows we will often denote as spectrum generators) have been developed in the past years to provide reliable determinations of the supersymmetric spectra in models with high-energy boundary conditions, including the calculations of the various radiative corrections. In particular, the publicly available codes include IsaJet [7], SoftSusy [8], SuSpect [9] and SPheno [10]. Detailed comparisons of the features and the results of these codes can be found in Ref. [11] for instance.

A crucial prediction of the MSSM is the existence of at least one light Higgs boson [12] which, at the tree level, is bound to be lighter than the $Z$ boson. If this upper bound was not significantly raised by radiative corrections, the failure of detecting this Higgs boson at LEP would have ruled out the MSSM as a viable theory for physics at the weak scale. However, it was first realised in Ref. [13] that the inclusion of the one-loop $\mathcal{O}\left(\alpha_{t}\right)$ corrections, which rise quartically with the mass of the top quark and logarithmically with the mass of its scalar superpartner, may push the lighter Higgs boson mass well above the tree-level bound. 
In the subsequent years, an impressive theoretical effort has been devoted to the precise determination of the Higgs boson masses in the MSSM. A first step was to provide the full one-loop computation, performed in Refs. [14, 15, 16]. A second step was the addition of the dominant two-loop corrections which involve the strongest couplings of the theory: the QCD coupling constant and the Yukawa couplings of the heavy third generation fermions (although the masses of the bottom quark and the $\tau$ lepton are relatively tiny compared to the top quark mass, the $b$ and $\tau$ Yukawa couplings can be strongly enhanced for large values of $\tan \beta$, the ratio of the vacuum expectation values of the two Higgs doublet fields).

The leading logarithmic effects at two loops have been subsequently included via appropriate RGEs [17, 18], and the genuine two-loop corrections of $\mathcal{O}\left(\alpha_{t} \alpha_{s}\right)$ [19, 20, 21, 22, 23] and $\mathcal{O}\left(\alpha_{b} \alpha_{s}\right)$ 24] have been evaluated in the limit of zero external momentum. The two-loop Yukawa corrections of $\mathcal{O}\left(\alpha_{t}^{2}\right)[19,22,25]$ and $\mathcal{O}\left(\alpha_{t} \alpha_{b}+\alpha_{b}^{2}\right)$ [26] have been also evaluated in the limit of zero external momentum. To complete the calculation of the two-loop corrections controlled by third-generation fermion couplings, only the expectedly small corrections that are proportional to the $\tau$-lepton Yukawa coupling are still to be determined. The $\mathcal{O}\left(\alpha_{\tau}^{2}\right)$ corrections can be straightforwardly derived from the $\mathcal{O}\left(\alpha_{t}^{2}\right)$ corrections as explained in Ref. [26]; the missing $\mathcal{O}\left(\alpha_{b} \alpha_{\tau}\right)$ contributions are provided in this paper.

The tadpole corrections needed to minimise the effective scalar potential, $V_{\text {eff }}$, have also been calculated at the one-loop [16, 27] and two-loop [26, 28] levels for the strong coupling and the top and bottom quark Yukawa couplings; the tadpole corrections involving the $\tau$-lepton Yukawa coupling will also be provided in this paper. Finally, the full twoloop corrections to the MSSM effective potential have been calculated [29], together with a first study of the two-loop corrections to the lighter Higgs boson mass controlled by the electroweak gauge couplings [30].

The calculation of the radiative corrections to physical observables requires the choice of a renormalisation scheme. For example, one might choose to express the corrections in terms of "On-Shell" (OS) parameters, such as pole particle masses and suitably defined mixing angles. This is the scheme adopted for the computation of the MSSM Higgs boson masses by the computer code FeynHiggs [31], based on the results of Refs. [15, 20] and subsequently expanded to include the results of Refs. [24, 25, 26, 32] (see Ref. [33] for a discussion).

However, when the MSSM parameters at the weak scale are derived from a set of unified parameters at the GUT scale via renormalisation group (RG) evolution, they come naturally as unphysical "running" quantities expressed in a minimal subtraction scheme such as the modified Dimensional Reduction scheme, $\overline{\mathrm{DR}}$, which is usually adopted since it preserves SUSY, at least up to two-loop order. Interfacing the output of the RG evolution of the MSSM parameters with a two-loop calculation of the Higgs boson masses based on the OS scheme requires translating the $\overline{\mathrm{DR}}$ input parameters into physical quantities, at the twoloop level for the parameters entering the tree-level mass matrix (such as the CP-odd Higgs boson mass) and at the one-loop level for the parameters entering the one-loop corrections (such as the squark masses and mixing angles). This induces additional complications in the procedure. 
A more direct strategy would be to perform the computation of the Higgs boson masses directly in the $\overline{\mathrm{DR}}$ renormalisation scheme, using the MSSM parameters as they come from the RG evolution. The results for the Higgs boson masses must be equivalent to those of the OS calculation up to terms that are formally of higher order in the perturbative expansion. The numerical differences between the results of the two calculations can be taken as an estimate of the size of the corrections that are still uncomputed. They can be viewed, together with the differences due to changes in the renormalisation scale at which the corrections are evaluated, as part of the theoretical uncertainty in the calculation.

A first purpose of this paper is to present the implementation of a purely $\overline{\mathrm{DR}}$ calculation of the neutral MSSM Higgs boson masses, based on the one-loop results of Ref. [16] and the two-loop results of Refs. [23, 25, 26, 28], into the latest versions of three public codes for the RG evolution of the MSSM parameters and the computation of the superparticle and Higgs boson mass spectrum, i.e. SoftSusy1.8.7, SPheno2.2.1 and SuSpect2.3. We also derive the small $\mathcal{O}\left(\alpha_{b} \alpha_{\tau}\right)$ contributions to complete the calculation of the two-loop corrections to the Higgs boson masses controlled by the third-generation Yukawa couplings.

A second purpose of this paper is to estimate the various theoretical uncertainties in the determination of the Higgs boson masses. Using representative choices for the high-energy boundary conditions, known as Snowmass Points and Slopes (SPS) [34, we first compare the outputs of the three codes, which are generally in very good agreement. We outline the reasons for the small residual discrepancies and briefly discuss the effect on the Higgs boson masses of various two-loop refinements of the general calculation. We then compare the results of the $\overline{\mathrm{DR}}$ computations of the Higgs masses with those of the OS computation in the program FeynHiggs and obtain an estimate of the residual scheme dependence. We also discuss the renormalisation scale dependence of our results for the physical masses, which can also be taken as an estimate of the theoretical uncertainty, and the effect of the approximation of zero external momentum in the two-loop corrections. A similar analysis is performed in the context of the unconstrained MSSM. Finally, we estimate the uncertainties in the Higgs boson masses arising from the experimental error in the determination of the top and bottom quark masses and the strong and electromagnetic coupling constants.

A final purpose of this paper is to study the impact of these results on MSSM Higgs phenomenology, under different assumptions for the mechanism of supersymmetry breaking. In particular, we estimate the upper bounds on the lighter Higgs boson mass in the general MSSM and in the mSUGRA, GMSB and AMSB scenarios once the latest values of the SM parameters (in particular the new experimental value for the top quark mass, $M_{t}=$ 178.0 $\pm 4.3 \mathrm{GeV}[35]$ ) are used and the theoretical and experimental uncertainties are included; the knowledge of the precise value of this bound might be crucial to discriminate various scenarios of SUSY-breaking. We also discuss the implications of the LEP2 constraints on the MSSM Higgs sector and derive a lower bound on the value of $\tan \beta$, once various sources of uncertainty are taken into account. We then compare our results with those of previous analyses [36, 37, 38], which employ different computations of the Higgs boson masses, different codes for the determination of the (s)particle spectrum and which include 
only a subset of the errors discussed in the present analysis.

The rest of the paper is organised as follows. In section 2, we describe the general structure of the spectrum generators, focusing on the aspects that are more critical to the mechanism of EWSB and the computation of the Higgs boson masses. In section 3 we compute the MSSM Higgs boson masses, compare the results from the three codes, and analyse the effect of various two-loop refinements, including the small effect of the $\mathcal{O}\left(\alpha_{b} \alpha_{\tau}\right)$ and $\mathcal{O}\left(\alpha_{\tau}^{2}\right)$ corrections. We also discuss the theoretical uncertainties associated with the scheme dependence (comparing the $\overline{\mathrm{DR}}$ and OS computations), the renormalisation scale dependence (variation with $M_{\mathrm{EWSB}}$ ), and the possible effect of the non-zero external momenta in the two-loop self-energies. In section 4 we study the impact of our results and the effects of the uncertainties on the upper bound on the lighter Higgs boson mass (and on its decay modes), as well as on the lower bound on the parameter $\tan \beta$, in various scenarios for the mechanism of SUSY breaking. We present our conclusions in section 5. Finally, in appendix A we provide for completeness the general formulae for the computation of the EWSB conditions and the Higgs masses, and in appendix B we provide explicit analytic formulae for the $\mathcal{O}\left(\alpha_{b} \alpha_{\tau}\right)$ corrections to the MSSM Higgs boson masses and tadpoles.

\section{Determining the MSSM mass spectrum}

In this section, we describe the calculation of the MSSM mass spectrum implemented in the latest versions of three publicly available spectrum generators, i.e. SoftSusy1.8.7, SPheno2.2.1 and SuSpect2.3. We start with the general algorithm used, then discuss some of the differences among the individual codes. The spectrum generator codes' manuals [8, 9, 10] describe in detail the approximations used to perform their calculations. Here, we simply discuss the aspects of the calculation that are more critical to the Higgs boson masses.

Assuming CP and R-parity conservation, the MSSM RGEs consist of some 110 coupled non-linear first-order homogeneous ordinary differential equations. The calculation of the MSSM mass spectrum consists of a two-boundary problem: we must solve these differential equations given boundary conditions at two values of the independent variable, the renormalisation scale $Q$. Evolution of these RGEs is a standard problem, and there exist many techniques to perform the task [39]. The high-scale (theoretical) boundary conditions are upon some of the parameters in the soft SUSY-breaking Lagrangian, and are specified by the model assumed for SUSY breaking (e.g., mSUGRA, AMSB, GMSB) and its parameters. They are often applied at the scale $M_{\mathrm{GUT}} \sim \mathcal{O}\left(10^{16}\right) \mathrm{GeV}$, at which the running electroweak gauge couplings unify:

$$
g_{1}\left(M_{\mathrm{GUT}}\right)=g_{2}\left(M_{\mathrm{GUT}}\right)
$$

where $g_{1} \equiv \sqrt{5 / 3} g^{\prime}$ and $g_{2} \equiv g$ with $g$ and $g^{\prime}$ the couplings associated with the $\mathrm{SU}(2)_{\mathrm{L}}$ and $\mathrm{U}(1)_{\mathrm{Y}}$ gauge groups of the MSSM, respectively. We do not enforce that the $\mathrm{SU}(3)_{\mathrm{C}}$ coupling constant $g_{s} \equiv g_{3}$ unifies with $g_{1}$ and $g_{2}$ at the high scale; the few percent discrepancy 
from real unification is assumed to be accounted for by threshold corrections at $M_{\mathrm{GUT}}$. A case apart is the GMSB model, in which the high-scale boundary conditions are set at the messenger scale $M_{\text {mess }}$.

The weak-scale boundary conditions set the gauge and Yukawa couplings by matching the running MSSM parameters to the experimental data at some renormalisation scale, usually taken as $Q=M_{Z}$. This step is quite involved, and requires subtracting the radiative corrections from the experimental data in order to arrive at the $\overline{\mathrm{DR}}$-renormalised MSSM parameters. Using the formulae of Ref. [16] and references therein, the MSSM $\overline{\mathrm{DR}}$ gauge couplings $g, g^{\prime}, g_{3}$ and the electroweak parameter $v$ can be computed at $Q=M_{Z}$ from a set of four experimental input parameters.

In the spirit of the SUSY Les Houches Accord (SLHA) for interfacing the spectrum generators with other computer codes [40], these input parameters can be chosen as: $G_{F}$, the Fermi constant determined from the muon decay; $M_{Z}$, the pole mass of the $Z$ boson; $\alpha_{\mathrm{em}}\left(M_{Z}\right)^{\overline{\mathrm{MS}}}$, the five-flavour SM electromagnetic coupling at the scale $M_{Z}$ in the $\overline{\mathrm{MS}}$ scheme; $\alpha_{s}\left(M_{Z}\right)^{\overline{\mathrm{MS}}}$, the five-flavour SM strong coupling at the scale $M_{Z}$ in the $\overline{\mathrm{MS}}$ scheme. In particular, the running parameters $g, g^{\prime}$ and $v$ are connected to the running $Z$-boson mass $m_{Z}$ by the relation:

$$
m_{Z}^{2}=M_{Z}^{2}+\operatorname{Re} \Pi_{Z Z}^{T}\left(M_{Z}^{2}\right)=\frac{1}{4}\left(g^{2}+g^{\prime 2}\right) v^{2},
$$

where $\Pi_{Z Z}^{T}\left(M_{Z}^{2}\right)$ is the transverse part of the $Z$ boson self-energy computed at a squared external momentum equal to the squared pole $Z$ boson mass.

The Yukawa couplings $h_{u}(u=u, c, t)$ for the up-type quarks, $h_{d}(d=d, s, b)$ for the down-type quarks and $h_{\ell}(\ell=e, \mu, \tau)$ for the leptons are determined from the corresponding running fermion masses as

$$
h_{u}=\frac{\sqrt{2} m_{u}}{v \sin \beta}, \quad h_{d}=\frac{\sqrt{2} m_{d}}{v \cos \beta}, \quad h_{\ell}=\frac{\sqrt{2} m_{\ell}}{v \cos \beta}
$$

where $\tan \beta \equiv v_{2} / v_{1}$ is the ratio of the vacuum expectation values (VEVs) of the two MSSM neutral Higgs fields, $H_{1}^{0}$ and $H_{2}^{0}$, that also obey the relation $v_{1}^{2}+v_{2}^{2}=v^{2}$.

The running fermion masses $m_{f}$ (with $f=u, d, \ell$ ) in eq. (3) can be derived at the one-loop level from the corresponding pole masses $M_{f}$, through the relation

$$
m_{f}=M_{f}+\Sigma_{f}\left(M_{f}\right)
$$

where $\Sigma_{f}\left(M_{f}\right)$ is the one-loop fermion self-energy computed at an external momentum equal to the pole mass. In the case of the top quark, the self-energy includes also the leading twoloop standard QCD corrections

$$
m_{t}=M_{t}+\Sigma_{t}\left(M_{t}\right)+\left(\Delta m_{t}\right)^{2-\text { loop }, \mathrm{QCD}}
$$

where the precise expression for $\left(\Delta m_{t}\right)^{2-\text { loop, QCD }}$ depends on the renormalisation scheme in which the parameters entering the one-loop self-energy $\Sigma_{t}$ are expressed. In the case of 
the bottom quark, the SLHA prescribes to take as input the SM running mass in the $\overline{\mathrm{MS}}$ scheme, $m_{b}\left(m_{b}\right)^{\overline{\mathrm{MS}}}$. Moreover, a "resummation" procedure is required (see e.g. Ref. [41]) in order to properly take into account the large QCD corrections, as well as the $\tan \beta$-enhanced SUSY corrections [42], to the relation between the input bottom mass and the corresponding MSSM, $\overline{\mathrm{DR}}$ Yukawa coupling. We extract the latter, via eq. (3) , from the MSSM, $\overline{\mathrm{DR}}$ bottom mass $\widehat{m}_{b}$, defined at the scale $Q=M_{Z}$ by the following matching condition:

$$
\widehat{m}_{b} \equiv m_{b}\left(M_{Z}\right)_{\mathrm{MSSM}}^{\overline{\mathrm{DR}}}=\frac{\bar{m}_{b}}{1-\Delta_{b}}
$$

where $\bar{m}_{b} \equiv m_{b}\left(M_{Z}\right)_{\mathrm{SM}}^{\overline{\mathrm{DR}}}$ is the SM, $\overline{\mathrm{DR}}$ bottom mass, obtained by evolving $m_{b}\left(m_{b}\right)^{\overline{\mathrm{MS}}}$ up to the scale $Q=M_{Z}$ with the appropriate RGE, in order to resum the QCD corrections, and then converting it to the $\overline{\mathrm{DR}}$ scheme; $\Delta_{b} \equiv \Sigma_{b}\left(\widehat{m}_{b}\right) / \widehat{m}_{b}$ accounts for the remaining non-gluonic corrections, some of which are enhanced by a factor $\tan \beta$. It has been shown [41] that defining the running MSSM bottom mass as in eq. (6) guarantees that the large threshold corrections of $\mathcal{O}\left(\alpha_{s} \tan \beta\right)^{n}$ are included in $\widehat{m}_{b}$ to all orders in the perturbative expansion. In the case of the $\tau$ lepton, the only $\tan \beta$-enhanced corrections to be included are those controlled by the electroweak gauge couplings, stemming from chargino-slepton loops.

The computation of the MSSM mass spectrum is also complicated by the requirement that the electroweak symmetry is broken radiatively: at some weak scale $M_{\mathrm{EWSB}}$, the Higgs VEVs $v_{1}$ and $v_{2}$ can be computed by the minimisation of the MSSM effective potential, $V_{\text {eff }}$. In principle, a set of high-energy boundary conditions is acceptable if, after RG evolution of the parameters down to $M_{\mathrm{EWSB}}$, it leads to the correct value of the squared running mass for the $Z$ boson, as given in eq. (2). In practice, however, it is more convenient to assume that there is successful electroweak symmetry breaking, and trade two of the high-energy input parameters for $v_{1}$ and $v_{2}$ (or, equivalently, for $v$ and $\tan \beta$ ). The minimisation conditions of the effective potential then allow one to determine the weak-scale values of two MSSM parameters, usually chosen as the Higgs mass term in the superpotential, $\mu$, and its soft SUSY-breaking counterpart, $B$, the latter being related to the squared running mass for the $\mathrm{CP}-$ odd Higgs boson $A, m_{A}$, through the relation

$$
m_{A}^{2}=2 B / \sin 2 \beta
$$

The computation of $\mu$ and $B$ beyond tree level requires the knowledge of the so-called tadpole corrections to the minimisation conditions of the effective potential. We provide the corresponding formulae in appendix A. At the one-loop level, SoftSusy, SPheno and SuSpect use the complete calculation of the tadpoles given in Ref. [16]. Among the twoloop corrections, the most relevant are those controlled by the top and bottom Yukawa couplings and by the strong gauge coupling. The three codes use the results of Ref. [28] for the $\mathcal{O}\left(\alpha_{t} \alpha_{s}+\alpha_{b} \alpha_{s}\right)$ corrections, and those of Ref. [26] for the $\mathcal{O}\left(\alpha_{t}^{2}+\alpha_{t} \alpha_{b}+\alpha_{b}^{2}\right)$ corrections. Besides being required by consistency with the Higgs boson mass calculations, these two-loop corrections are relevant to stabilise the scale dependence of the MSSM parameters obtained 
from the minimisation conditions of the effective potential (see the discussion in Ref. [28]). Note that also for consistency reasons, the Higgs mass parameters $m_{H_{1}}^{2}$ and $m_{H_{2}}^{2}$ which enter the minimisation conditions on $V_{\text {eff }}$ have to be evolved using the two-loop RGEs.

After determining the full set of MSSM parameters, expressed in the $\overline{\mathrm{DR}}$ renormalisation scheme at the scale $M_{\mathrm{EWSB}}$, the codes compute the spectrum of physical masses of the supersymmetric particles. The approximations employed in the computation of sfermion, gluino, chargino and neutralino physical masses differ from code to code, and we refer readers interested in the details to the respective manuals.

On the other hand, due to extreme phenomenological relevance of the mass spectrum of the MSSM Higgs sector (as the failure of detecting a light Higgs boson already rules out a considerable fraction of the MSSM parameter space), it is mandatory to employ a precise calculation of the neutral Higgs boson masses that encompasses all the presently available radiative corrections. The general formulae for the computation of the Higgs masses are provided for completeness in appendix A. For the analysis presented in this paper, the spectrum generators SoftSusy, SPheno and SuSpect have been upgraded to include the state-of-the-art radiative corrections in the Higgs sector.

In the newest versions SoftSusy1.8.7, SPheno2.2.1 and SuSpect2.3 the complete oneloop formulae, including the tadpole corrections, are taken from Ref. [16], which also provides formulae for the one-loop corrections to the charged Higgs boson mass. Concerning the twoloop part, the three codes employ the effective potential results of Ref. [23] for the $\mathcal{O}\left(\alpha_{t} \alpha_{s}\right)$ corrections, and those of Ref. [26] for the $\mathcal{O}\left(\alpha_{t}^{2}+\alpha_{t} \alpha_{b}+\alpha_{b}^{2}\right)$ corrections. The formulae for the $\mathcal{O}\left(\alpha_{b} \alpha_{s}\right)$ corrections are obtained from those for the $\mathcal{O}\left(\alpha_{t} \alpha_{s}\right)$ corrections with appropriate replacements. The two-loop corrections are computed in the $\overline{\mathrm{DR}}$ renormalisation scheme, i.e. they require that the one-loop part of the corrections is expressed in terms of running, $\overline{\mathrm{DR}}-$ renormalised MSSM parameters. This is a particularly convenient scheme in the situation in which the MSSM parameters are computed via RG evolution from a set of unified highenergy boundary conditions.

The two-loop corrections to the Higgs boson masses controlled by the third-family Yukawa couplings can be completed by including the contribution of the $\tau$ Yukawa coupling. The inclusion of the $\mathcal{O}\left(\alpha_{\tau}^{2}\right)$ corrections was discussed in Ref. [26], and in appendix B we present a new computation of the remaining corrections of $\mathcal{O}\left(\alpha_{b} \alpha_{\tau}\right)$. However, we found that the numerical impact of the $\tau$ radiative corrections is very small in all the scenarios that we considered in our analysis.

Other efforts to improve the two-loop calculation of the Higgs boson masses include an effective potential computation of the corrections to the lighter Higgs boson mass controlled by the electroweak couplings [30], and a first attempt to go beyond the effective potential approximation by including the effect of non zero external momenta in the two-loop propagators [43]. Two-loop corrections to the charged Higgs boson mass should also be calculated. We plan to include these results, as soon as they become available in a suitable form, in future updates of our spectrum generators. 
It appears from the discussion above that the problem of determining the MSSM mass spectrum involves several mass scales, at which boundary conditions and EWSB conditions are imposed. As the computation of the radiative corrections at some scale requires knowledge about parameters that are determined at a different scale, an iterative procedure is adopted. The algorithm adopted by the codes can be summarised as follows:

1. An initial guess for the MSSM parameters is taken at $Q=M_{Z}$.

2. The parameters are then evolved to $M_{\mathrm{GUT}}$ (or $M_{\text {mess }}$ in the GMSB case), where the high scale boundary conditions are imposed.

3. The new set of MSSM parameters is then evolved to $M_{\mathrm{EWSB}}$, where the EWSB constraints are imposed.

4. The MSSM pole mass spectrum is calculated.

5. The parameters are then evolved to $Q=M_{Z}$, where the Yukawa and gauge couplings are matched to empirical data.

6. One then proceeds to step 2, continuing until the MSSM parameters converge upon stable values.

Although the three codes employ the same general procedure in the determination of the MSSM mass spectrum, they differ in many details when it comes to the implementation of the single steps of the calculation. In particular, the differences that most affect the results for the Higgs boson masses are:

- SPheno uses two-loop RGE for all the MSSM parameters, whereas SoftSusy and SuSpect use one-loop RGE for the soft SUSY-breaking masses and interaction terms of the sfermions (and two-loop RGE for the remaining MSSM parameters);

- SoftSusy and SPheno include by default in the RGE the contributions of the Yukawa couplings for the three generations of fermions, whereas SuSpect works under the approximation that the Yukawa couplings of the first two generations are zero. However, we found that the omission of the first two-family Yukawa couplings has always a very tiny impact on the Higgs boson masses, which is limited to the case of large $\tan \beta$;

- SPheno defines the default EWSB scale $M_{\mathrm{EWSB}}=\sqrt{m_{\tilde{t}_{1}} m_{\tilde{t}_{2}}}$ in terms of the physical stop masses, whereas SoftSusy and SuSpect define it in terms of the running stop masses (note, however, that all the codes also allow for an arbitrary choice of $M_{\mathrm{EWSB}}$ );

- when computing the running top mass at the scale $Q=M_{Z}$ according to eq. (5), SPheno uses the running top mass itself in the one-loop part of the top self-energy, whereas SoftSusy and SuSpect use the pole top mass. This induces a difference in the formulae for the two-loop QCD corrections, which is properly taken into account by the codes; 
- when computing the running bottom mass at the scale $Q=M_{Z}$ according to eq. (6), SPheno defines $\Delta_{b}$ in terms of the full bottom self-energy, whereas SoftSusy and SuSpect include only the $\tan \beta$-enhanced contributions;

- when computing the threshold corrections to the Yukawa couplings at the scale $Q=$ $M_{Z}$, SPheno uses the pole top mass in the $Z$ boson self energy (required to obtain the running EWSB parameter $v$, see eq. (2)) whereas SoftSusy and SuSpect use the running top mass;

- in the computation of the various threshold corrections at the scale $Q=M_{Z}$, SoftSusy and SPheno use for all the sparticle masses and mixing angles the $\overline{\mathrm{DR}}$ running values computed at $Q=M_{Z}$, whereas SuSpect uses the values computed at $M_{\text {EwsB }}$ for the neutralino and chargino masses and mixing angles;

- in the computation of the various radiative corrections at the scale $M_{\mathrm{EWSB}}$, SPheno and SuSpect compute $v\left(M_{\mathrm{EWSB}}\right)$ through eq. (2), whereas SoftSusy takes the value computed at the scale $Q=M_{Z}$ and evolves it up to $M_{\mathrm{EWSB}}$ with the appropriate RGE.

All of the differences listed above correspond to effects that are of higher order with respect to the accuracy required by the calculation of the Higgs boson masses. However, as will be discussed in the next section, they induce non negligible variations in the results for the Higgs boson masses, amounting to something less than a GeV for the lighter Higgs boson and a few $\mathrm{GeV}$ for the heavier Higgs bosons. For the time being, such variations must be taken as contributing to the uncertainties that still affect the calculation. Only when more precise computations of the radiative corrections become available will it be possible to fix the procedural ambiguities and reduce the uncertainty in the results.

Before presenting our numerical results in the next section, we point out that the precise determination of the Higgs boson masses that we are discussing here does not only apply in constrained models such as mSUGRA, GMSB and AMSB. The neutral Higgs boson masses can also be determined in a general (unconstrained) MSSM, where the soft SUSY-breaking parameters are set by hand at the weak scale. The three spectrum generators have options in which such a procedure can be applied (although in the case of SoftSusy the procedure is not yet completely implemented). The programs can therefore also be viewed as $\overline{\mathrm{DR}}$ Higgs boson mass calculators in a general MSSM framework, analogous to the FeynHiggs calculation in the On-Shell scheme 1 . At the end of the next section, we will present some illustrations of the calculation of the Higgs boson masses in the general or unconstrained MSSM.

\footnotetext{
${ }^{1}$ In fact, some of us are planning to include the part of the spectrum generators concerning the Higgs sector into the program HDECAY 44] which evaluates the Higgs boson decay widths and branching ratios in the SM and in the MSSM.
} 


\section{Precise results for the MSSM Higgs boson masses}

In this section we discuss in detail the results of the two-loop $\overline{\mathrm{DR}}$ computation of the Higgs boson masses and of the EWSB conditions in the constrained MSSM (cMSSM), focusing on the mSUGRA, GMSB and AMSB models for SUSY breaking; we also display some results in the unconstrained MSSM. In a first part, we specify the physical scenarios in which we work. We then compare the results of the three codes, SoftSusy, SPheno and SuSpect, in the case of the cMSSM and discuss the impact of the various radiative corrections that affect the Higgs boson masses and the EWSB conditions. We subsequently discuss the various theoretical uncertainties which affect the Higgs boson mass calculations: the scheme dependence, the renormalisation scale dependence and the effect of the external momenta in the two-loop self-energies. We finally summarise the theoretical uncertainty on the Higgs boson mass determinations and discuss the impact of experimental errors on the SM input parameters on these calculations.

\subsection{The physical scenarios}

We will first work in the framework of constrained MSSM scenarios and illustrate our results in the case of the mSUGRA, GMSB and AMSB models for SUSY-breaking. For what concerns the choice of the high-energy boundary conditions, we restrict ourselves to six (out of ten) Snowmass Points and Slopes (SPS) [34]. Four of these points are mSUGRA ones, where the relevant input parameters are three universal soft SUSY-breaking terms, $m_{0}, m_{1 / 2}$ and $A_{0}$, the value of $\tan \beta$ (expressed in the $\overline{\mathrm{DR}}$ scheme at the renormalisation scale $Q=M_{Z}$ ) and the scale-invariant sign of $\mu$ :

$\begin{array}{lclcl}\text { SPS 1a }: & m_{0}=100 \mathrm{GeV}, & m_{1 / 2}=250 \mathrm{GeV}, & A_{0}=-100 \mathrm{GeV}, & \tan \beta=10, \quad \mu>0, \\ \text { SPS 2: } & m_{0}=1450 \mathrm{GeV}, & m_{1 / 2}=300 \mathrm{GeV}, & A_{0}=0, & \tan \beta=10, \quad \mu>0, \\ \text { SPS 4: } & m_{0}=400 \mathrm{GeV}, & m_{1 / 2}=300 \mathrm{GeV}, & A_{0}=0, & \tan \beta=50, \quad \mu>0, \\ \text { SPS 5 : } & m_{0}=150 \mathrm{GeV}, & m_{1 / 2}=300 \mathrm{GeV}, & A_{0}=-1 \mathrm{TeV}, & \tan \beta=5, \quad \mu>0 .\end{array}$

The first choice, SPS1a, corresponds to a "standard" mSUGRA scenario; the second choice, SPS2, is called a "focus point" scenario and has interesting implications for dark matter abundance; SPS4 is characterised by a large value of $\tan \beta$ while SPS5 is characterised by a large value of the stop mixing parameter and leads to a light stop squark. The focus point and the large $\tan \beta$ scenario are known to be the most sensitive to the various approximations made by the spectrum generators [11].

We will also choose one point in the GMSB scenario, where the input parameters are the SUSY-breaking scale $\Lambda$, the messenger scale $M_{\text {mess }}$, the messenger index $N_{\text {mess }}$, $\tan \beta$ and the sign of $\mu$ : the SPS8 point in which the lightest SUSY particle is the lightest neutralino

$$
\text { SPS 8 : } \Lambda=100 \mathrm{TeV}, \quad M_{\text {mess }}=200 \mathrm{TeV}, \quad N_{\text {mess }}=1, \quad \tan \beta=15, \quad \mu>0 .
$$


The last point, denoted SPS9, is for an AMSB scenario described by a large gravitino mass $m_{3 / 2}$, a common mass term for the scalars $m_{0}, \tan \beta$ and the sign of $\mu$ :

$$
\text { SPS } 9: m_{3 / 2}=60 \mathrm{TeV}, m_{0}=450 \mathrm{GeV}, \tan \beta=10, \mu>0 \text {. }
$$

We remark in passing that in Ref. [34] the SPS scenarios are defined in terms of the low-energy MSSM $\overline{\mathrm{DR}}$ parameters, as computed by the code IsaJet7.58 at the weak scale $M_{\text {EWsB }}$. Thus, calling "SPS scenarios" the above choices of high-energy boundary conditions always involves a slight abuse of language.

In a next step, we will work in the case of the unconstrained MSSM. For the latter scenario, we take the so-called "phenomenological MSSM" (pMSSM) [2], in which some constraints have been imposed (such as CP conservation, flavour diagonal sfermion mass and coupling matrices and universality of the first and second generations). These constraints ensure that an appreciable fraction of parameter space of the pMSSM has viable phenomenology. The model involves 22 free parameters in addition to those of the SM:

- the parameter $\tan \beta$;

- the two soft SUSY-breaking Higgs mass parameters $m_{H_{1}}^{2}$ and $m_{H_{2}}^{2}$, which can be traded against $M_{A}$ and $\mu$ by enforcing the EWSB conditions;

- the three gaugino mass parameters $M_{1}, M_{2}$ and $M_{3}$;

- the diagonal sfermion mass parameters $m_{\tilde{f}_{L, R}}$ : five for the third generation sfermions and five others for the first/second generation sfermions;

- the trilinear sfermion couplings $A_{f}$ : three for the third generation sfermions and three others for the first/second generation sfermions.

Fortunately, most of these parameters have only a marginal impact on the Higgs boson masses, a fact which considerably simplifies the analysis. A very important parameter in the context of the radiative corrections to the MSSM Higgs boson masses is the mixing parameter in the stop sector, $X_{t}=A_{t}-\mu \cot \beta$. For our numerical illustrations, we will consider three pMSSM scenarios, in which the SUSY spectrum is rather heavy: the soft SUSY-breaking masses of the sfermions (all set equal to a common mass $M_{S}$ ), the $\mathrm{SU}(3$ ) gaugino mass $M_{3}$ ( $M_{1}$ and $M_{2}$ being related to the latter through the usual GUT relation), the pseudoscalar Higgs boson mass $M_{A}$ and the higgsino mass parameter $\mu$ are set to $1 \mathrm{TeV}$; for $\tan \beta$ we choose a moderate value, $\tan \beta=10$; furthermore, we consider three cases for the mixing $X_{t}$ in the stop sector: no mixing, $X_{t}=0$, typical mixing, $X_{t}=M_{S}$, and large mixing, $X_{t}=\sqrt{6} M_{S}$ (the other sfermion trilinear couplings will be also set to $1 \mathrm{TeV}$ ). All of the MSSM input parameters are taken as $\overline{\mathrm{DR}}$ running quantities computed at the scale $Q=1 \mathrm{TeV}$, apart from $\tan \beta$ which is computed at $Q=M_{Z}$, and $M_{A}$ which denotes the physical mass. Thus we will have three pMSSM points:

$$
\begin{array}{ll}
\text { pMSSM1 : } & M_{S}=A_{b, \tau}=M_{3}=M_{A}=\mu=1 \mathrm{TeV}, \tan \beta=10, X_{t}=0, \\
\text { pMSSM2 : } & M_{S}=A_{b, \tau}=M_{3}=M_{A}=\mu=1 \mathrm{TeV}, \tan \beta=10, X_{t}=M_{S}, \\
\text { pMSSM3 : } & M_{S}=A_{b, \tau}=M_{3}=M_{A}=\mu=1 \mathrm{TeV}, \tan \beta=10, X_{t}=\sqrt{6} M_{S} .
\end{array}
$$


The first and third of these scenarios are rather close to those which have been used as benchmark points in the interpretation in the unconstrained MSSM of the Higgs boson searches by the LEP2 collaborations [45].

Finally, the SM input parameters at the weak scale are fixed according to the SLHA prescription. In particular, we take for the electroweak and strong parameters [46]:

$$
\begin{gathered}
G_{F}=1.1663910^{-5} \mathrm{GeV}^{-2}, \quad M_{Z}=91.1876 \mathrm{GeV}, \\
\alpha_{\mathrm{em}}^{-1}\left(M_{Z}\right)^{\overline{\mathrm{MS}}}=127.934 \pm 0.027, \quad \alpha_{s}\left(M_{Z}\right)^{\overline{\mathrm{MS}}}=0.1172 \pm 0.002
\end{gathered}
$$

and for the third-generation fermion masses the values [35, 46]:

$$
M_{t}=178.0 \pm 4.3 \mathrm{GeV}, \quad m_{b}\left(m_{b}\right)^{\overline{\mathrm{MS}}}=4.25 \pm 0.25 \mathrm{GeV}, \quad M_{\tau}=1.777 \mathrm{GeV} .
$$

In most of our discussion we take the SM input parameters to be equal to their central values (the errors on $G_{F}, M_{Z}$ and $M_{\tau}$ are indeed very small and we omit them in the equations above). However, in section 3.6 we discuss the uncertainty in the determination of the Higgs masses arising from the experimental errors on some of these parameters.

\subsection{Determination of the neutral Higgs masses in the cMSSM}

\subsubsection{Comparing the results of the three codes}

We start our discussion by comparing the values of the neutral Higgs boson masses and of the superpotential parameter $\mu$ in the six SPS scenarios discussed above, as they result from the computations of SoftSusy, SPheno and SuSpect at the default renormalisation scale $M_{\mathrm{EWSB}}=\sqrt{m_{\tilde{t}_{1}} m_{\tilde{t}_{2}}}$, once all of the two-loop radiative corrections are implemented. The corresponding values for the physical masses of the CP-even Higgs bosons, $M_{h}$ and $M_{H}$, for the physical mass of the $\mathrm{CP}$-odd Higgs boson, $M_{A}$, and for the parameter $\mu$ (the latter interpreted as a $\overline{\mathrm{DR}}$ running parameter computed at $M_{\mathrm{EWSB}}$ ) are given in the tables 1, 2, 3, and 4, respectively.

Concerning the lighter $\mathrm{CP}$-even Higgs boson mass, $M_{h}$, it can be seen that the agreement between the three codes is very good, the discrepancies being contained in a half $\mathrm{GeV}$. In the cases of the heavier Higgs boson masses and of $\mu$, which are more sensitive than $M_{h}$ to small variations in the RG evolution of the soft SUSY-breaking parameters, the discrepancies amount to at most few $\mathrm{GeV}$, generally below the $1 \%$ level. We find this agreement very satisfactory, taking into account the fact that the results are obtained with three independent codes that - although based on the same set of formulae for the corrections to the Higgs boson masses and the EWSB conditions - differ in many details of the calculation, as outlined in the previous section.

We have checked that, if we force the three codes to use the same computation of the threshold corrections to the gauge and Yukawa couplings and the same set of RGE, the residual discrepancies in the results for the neutral Higgs boson masses and $\mu$ become negligible. However, we stress again that the differences between the three codes are a matter 
of choice, because they all correspond to effects that are of higher order with respect to the accuracy required by the calculation. The spread in the values of the Higgs boson masses and $\mu$ resulting from tables 14 has to be considered as part of the theoretical uncertainty, and will be reduced only when more refined calculations of the radiative corrections become available.

\begin{tabular}{|l|c|c|c|c|c|c|}
\hline Code & SPS1a & SPS2 & SPS4 & SPS5 & SPS8 & SPS9 \\
\hline SoftSusy & 112.1 & 116.8 & 114.1 & 116.3 & 115.4 & 117.4 \\
SPheno & 112.2 & 117.1 & 114.3 & 116.5 & 115.8 & 117.8 \\
SuSpect & 112.1 & 116.8 & 114.1 & 116.1 & 115.5 & 117.5 \\
\hline
\end{tabular}

Table 1: The lighter CP-even Higgs boson mass, $M_{h}$, in the six SPS scenarios, as computed by SoftSusy1.8.7, SPheno2.2.1 and SuSpect2.3. The SM input parameters are chosen as in eqs. (8) $-(9)$.

\begin{tabular}{|l|c|c|c|c|c|c|}
\hline Code & SPS1a & SPS2 & SPS4 & SPS5 & SPS8 & SPS9 \\
\hline SoftSusy & 406.5 & 1553.0 & 355.8 & 686.8 & 550.4 & 1056.9 \\
SPheno & 406.0 & 1554.6 & 360.5 & 686.5 & 552.4 & 1051.1 \\
SuSpect & 406.5 & 1552.1 & 355.3 & 686.9 & 550.6 & 1056.6 \\
\hline
\end{tabular}

Table 2: Same as table 1 for the heavier CP-even Higgs boson mass, $M_{H}$.

\begin{tabular}{|l|c|c|c|c|c|c|}
\hline Code & SPS1a & SPS2 & SPS4 & SPS5 & SPS8 & SPS9 \\
\hline SoftSusy & 406.2 & 1552.9 & 355.8 & 687.0 & 550.1 & 1056.8 \\
SPheno & 405.7 & 1554.5 & 360.5 & 686.9 & 552.1 & 1051.0 \\
SuSpect & 406.1 & 1552.0 & 355.3 & 687.2 & 550.3 & 1056.5 \\
\hline
\end{tabular}

Table 3: Same as table 1 for the $\mathrm{CP}$-odd Higgs boson mass, $M_{A}$.

\begin{tabular}{|l|c|c|c|c|c|c|}
\hline Code & SPS1a & SPS2 & SPS4 & SPS5 & SPS8 & SPS9 \\
\hline SoftSusy & 364.8 & 586.5 & 413.8 & 631.2 & 440.1 & 1011.8 \\
SPheno & 364.3 & 588.2 & 414.7 & 631.2 & 442.2 & 1005.9 \\
SuSpect & 364.7 & 583.6 & 413.6 & 631.3 & 440.3 & 1011.1 \\
\hline
\end{tabular}

Table 4: Same as table 1 for the superpotential Higgs mass parameter $\mu$, expressed in the $\overline{\mathrm{DR}}$ renormalisation scheme at the scale $M_{\mathrm{EWSB}}$. 


\subsubsection{Impact of the radiative corrections}

We now discuss the importance of the various radiative corrections to the Higgs boson masses and tadpoles that are taken into account by the codes SoftSusy, SPheno and SuSpect. Tables 5 and 6 show the variations of $M_{h}$ and $M_{H}$, respectively, in the six SPS scenarios, as a result of different approximations for the radiative corrections. The first three rows of each table contain the values of the masses as obtained by SuSpect employing, respectively, the tree-level, one-loop and two-loop formulae for the Higgs masses and EWSB conditions. The variations shown in the second part of the tables result from progressively switching on the various two-loop corrections to the Higgs boson masses and EWSB conditions. Explicitly, we examine the two loop $\mathcal{O}\left(\alpha_{t} \alpha_{s}+\alpha_{b} \alpha_{s}\right)$ corrections (first line), the $\mathcal{O}\left(\alpha_{t}^{2}+\alpha_{t} \alpha_{b}+\alpha_{b}^{2}\right)$ corrections (second line) and the newly calculated $\mathcal{O}\left(\alpha_{\tau}^{2}+\alpha_{b} \alpha_{\tau}\right)$ corrections (third line).

\begin{tabular}{|l|c|c|c|c|c|c|}
\hline Approximation & SPS1a & SPS2 & SPS4 & SPS5 & SPS8 & SPS9 \\
\hline Tree-level & 88.6 & 87.8 & 90.2 & 82.9 & 89.2 & 88.2 \\
One-loop & 109.7 & 112.3 & 111.1 & 113.0 & 111.3 & 112.6 \\
Two-loop & 112.1 & 116.8 & 114.1 & 116.1 & 115.5 & 117.5 \\
\hline $\mathcal{O}\left(\alpha_{t} \alpha_{s}+\alpha_{b} \alpha_{s}\right)$ & +3.1 & +5.7 & +3.8 & +3.0 & +5.3 & +5.8 \\
$\mathcal{O}\left(\alpha_{t}^{2}+\alpha_{t} \alpha_{b}+\alpha_{b}^{2}\right)$ & -0.6 & -1.1 & -0.8 & +0.2 & -1.1 & -1.0 \\
$\mathcal{O}\left(\alpha_{\tau}^{2}+\alpha_{b} \alpha_{\tau}\right)$ & $<10^{-3}$ & $<10^{-3}$ & $<10^{-3}$ & $<10^{-3}$ & $<10^{-3}$ & $<10^{-3}$ \\
\hline
\end{tabular}

Table 5: The lighter CP-even Higgs boson mass, $M_{h}$, in the six SPS scenarios, as computed by SuSpect under different approximations for the radiative corrections. The first three rows contain the mass (in $\mathrm{GeV}$ ) computed at tree-level, one- and two-loop, respectively; the last three rows contain the shifts (in $\mathrm{GeV}$ ) due to the different two-loop contributions.

\begin{tabular}{|l|c|c|c|c|c|c|}
\hline Approximation & SPS1a & SPS2 & SPS4 & SPS5 & SPS8 & SPS9 \\
\hline Tree-level & 402.5 & 1542.7 & 356.3 & 689.6 & 525.7 & 1043.1 \\
One-loop & 406.8 & 1551.3 & 356.0 & 688.2 & 549.0 & 1056.1 \\
Two-loop & 406.5 & 1552.1 & 355.3 & 686.9 & 550.6 & 1056.6 \\
\hline $\mathcal{O}\left(\alpha_{t} \alpha_{s}+\alpha_{b} \alpha_{s}\right)$ & -0.1 & +1.1 & -0.1 & -0.9 & +2.5 & +0.9 \\
$\mathcal{O}\left(\alpha_{t}^{2}+\alpha_{t} \alpha_{b}+\alpha_{b}^{2}\right)$ & -0.3 & -0.3 & -0.5 & -0.3 & -0.9 & -0.4 \\
$\mathcal{O}\left(\alpha_{\tau}^{2}+\alpha_{b} \alpha_{\tau}\right)$ & $<10^{-3}$ & $<10^{-3}$ & 0.01 & $<10^{-3}$ & $<10^{-3}$ & $<10^{-3}$ \\
\hline
\end{tabular}

Table 6: Same as table 5 for the heavier $\mathrm{CP}$-even Higgs boson mass, $M_{H}$

Comparing the first three rows of table 5 it can be seen that the one-loop corrections to $M_{h}$ are positive and of the order of $20 \mathrm{GeV}$, while the two-loop corrections to $M_{h}$ are also positive and of the order of $2-5 \mathrm{GeV}$. This is to be contrasted with the case of the OS calculations (see e.g. Ref. [25]), where both the one-loop and two-loop corrections are 
usually larger, and the two-loop corrections are negative. However, as will be discussed in section 3.3.1, the two-loop results of the $\mathrm{OS}$ and $\overline{\mathrm{DR}}$ calculations agree within $2 \mathrm{GeV}$ in general. From the first three rows of table 6, instead, it can be seen that the one-loop corrections to $M_{H}$ amount in general to few $\mathrm{GeV}$, and their impact is usually of the order of $1 \%$ (apart from the scenario SPS8, where they reach 4\%). The two-loop corrections are also small, being of the order of one $\mathrm{GeV}$ or less. This is due to the fact that, in the considered scenarios, the heavier Higgs boson masses are large already at the tree-level, so that the inclusion of radiative corrections can have only a small effect.

Further information on the relative size of the various two-loop corrections to the Higgs boson masses can be obtained from the lower parts of tables 5 and 6. In the case of $M_{h}$, we see that the $\mathcal{O}\left(\alpha_{t} \alpha_{s}+\alpha_{b} \alpha_{s}\right)$ corrections, controlled by the strong gauge coupling, are dominant (amounting to $3-6 \mathrm{GeV}$ ) and positive, whereas the $\mathcal{O}\left(\alpha_{t}^{2}+\alpha_{t} \alpha_{b}+\alpha_{b}^{2}\right)$ corrections, involving only the top and bottom Yukawa coupling, are smaller (of the order of one $\mathrm{GeV}$ ) and usually negative (apart from the scenario SPS5). This is again to be contrasted with the case of the OS calculations, where the $\mathcal{O}\left(\alpha_{t} \alpha_{s}\right)$ corrections, which are always leading at twoloop order, are negative, and the subleading $\mathcal{O}\left(\alpha_{t}^{2}\right)$ corrections are positive (in both OS and $\overline{\mathrm{DR}}$ calculations, the corrections controlled by the bottom Yukawa coupling are negligible for small or moderate values of $\tan \beta$ ). In the case of $M_{H}$, instead, the trend is less definite: the $\mathcal{O}\left(\alpha_{t} \alpha_{s}+\alpha_{b} \alpha_{s}\right)$ corrections can be either positive or negative in the different scenarios, and they are not always larger than the $\mathcal{O}\left(\alpha_{t}^{2}+\alpha_{t} \alpha_{b}+\alpha_{b}^{2}\right)$ ones.

Concerning the corrections controlled by the $\tau$ Yukawa coupling, it can be seen that their effect is usually negligible, amounting to less than a $\mathrm{MeV}$ for both $M_{h}$ and $M_{H}$. Only in the scenario SPS4, in which the $\tau$ Yukawa coupling is enhanced by a large value of $\tan \beta$, a very tiny $(10 \mathrm{MeV})$ effect is visible in the heavier Higgs boson mass. This suppression of the $\tau$ corrections even in the case of large $\tan \beta$ is explained by the smallness of $m_{\tau}$ (even in comparison with $m_{b}$ ), by the absence of colour enhancements, and by the fact that the only $\tan \beta$-enhanced threshold corrections to the relation between $h_{\tau}$ and $m_{\tau}$ are those controlled by the electroweak gauge couplings.

One has to bear in mind, however, that all of the above considerations on the relevance of the various two-loop corrections to $M_{h}$ and $M_{H}$ depend to some extent on the choice of the electroweak symmetry breaking scale, taken by default as $M_{\mathrm{EWSB}}=\sqrt{m_{\tilde{t}_{1}} m_{\tilde{t}_{2}}}$. For example, as will be clear from the figures in section 3.3.2, with the choice $M_{\mathrm{EWSB}}=M_{Z}$ the two-loop corrections to $M_{h}$ become smaller $(1-2 \mathrm{GeV})$ and negative, while the two-loop corrections to $M_{H}$ amount to several GeV and they are positive. Also, in the scenario SPS4 the corrections to $M_{H}$ controlled by the $\tau$ Yukawa coupling get somewhat larger $(\sim 0.2 \mathrm{GeV})$. It is thus clear that - although it is always possible to choose $M_{\mathrm{EWSB}}$ in such a way that some of the two-loop corrections are small - only the proper inclusion of all of the leading two-loop corrections to the Higgs boson masses allows us to obtain reliable results that do not depend strongly on a preconceived choice of the renormalisation scale. 


\subsubsection{Impact of some higher-order effects}

To understand the importance of several higher-order effects that are taken into account by SoftSusy, SPheno and SuSpect, it may be useful to discuss their individual impact on the numerical values of the Higgs masses.

\begin{tabular}{|l|c|c|c|c|c|c|}
\hline Approximation & SPS1a & SPS2 & SPS4 & SPS5 & SPS8 & SPS9 \\
\hline Default & 112.17 & 117.06 & 114.34 & 116.46 & 115.80 & 117.82 \\
\hline 2-loop sfermion RGE & 0.07 & 0.15 & 0.04 & 0.03 & 0.03 & 0.03 \\
No $\Delta m_{t}^{\text {2loop }}$ & 0.26 & 0.33 & 0.28 & 0.35 & 0.35 & 0.29 \\
No $\Delta m_{t}^{\text {EW }}$ & 0.14 & 0.40 & 0.50 & 0.15 & 0.44 & 0.29 \\
No $m_{b}$ resummation & $<10^{-3}$ & $<10^{-3}$ & 0.06 & $<10^{-3}$ & $<10^{-3}$ & $<10^{-3}$ \\
Pole Higgs masses in 1-loop & 0.02 & 0.02 & 0.01 & 0.01 & 0.02 & 0.02 \\
\hline
\end{tabular}

Table 7: Lighter CP-even Higgs mass, $M_{h}$, in the six SPS scenarios, as computed by SPheno under different approximations for the higher-order effects. The first row contains the default values in $\mathrm{GeV}$, the other rows contain the shifts (in $\mathrm{GeV}$ ) due to the approximations.

\begin{tabular}{|l|c|c|c|c|c|c|}
\hline Approximation & SPS1a & SPS2 & SPS4 & SPS5 & SPS8 & SPS9 \\
\hline Default & 406.0 & 1554.6 & 360.5 & 686.5 & 552.4 & 1051.1 \\
\hline 2-loop sfermion RGE & 1.1 & 6.8 & 0.3 & -1.3 & 0.7 & 1.5 \\
No $\Delta m_{t}^{2 \text { 2loop }}$ & 1.1 & 6.7 & 4.5 & -1.4 & 2.6 & -2.1 \\
No $\Delta m_{t}^{\text {EW }}$ & 0.6 & 8.2 & 8.0 & -0.6 & 3.2 & -2.2 \\
No $m_{b}$ resummation & 0.2 & 0.3 & 31.0 & 0.1 & 0.3 & $<10^{-2}$ \\
Pole Higgs masses in 1-loop & 0.1 & 0.1 & 0.5 & 0.1 & 0.3 & 0.1 \\
\hline
\end{tabular}

Table 8: Same as table 7 for the heavier CP-even Higgs boson mass, $M_{H}$.

Tables 7 and 8 show the variations of $M_{h}$ and $M_{H}$, respectively, in the six SPS scenarios, as a result of different approximations for the higher-order corrections that affect the RGE evolution of the MSSM parameters. The first row of each table contains the default values of the masses, as obtained with the public version of SPheno2.2.1. In the first row of the second part of the table, we show the effect (in GeV) from switching off the two-loop terms in the RGEs for the sfermion soft SUSY-breaking parameters; in the second row, from switching off the two-loop QCD corrections in the computation of the top Yukawa coupling $h_{t}$; in the third, from switching off the one-loop electroweak corrections in the computation of $h_{t}$; in the fourth, from defining the running bottom mass as $\widehat{m}_{b}=\bar{m}_{b}\left(1+\Delta_{b}\right)$ instead of adopting the "resummed" formula of eq. (6); ; in the fifth, from using the physical values of the Higgs masses, instead of the running values, as input parameters for the computation of the one-loop corrections. 
We see that the effect of these approximations on $M_{h}$ is in general moderate, amounting at most to some tenths of a $\mathrm{GeV}$, whereas their effect on $M_{H}$ can amount to many $\mathrm{GeV}$. This is due to the fact that for $M_{A} \gg M_{Z}$, as is always the case in the SPS scenarios, the tree-level value of $M_{h}$ is essentially proportional to $m_{Z}$, and the dependence on the MSSM parameters enters only through the radiative corrections. On the other hand, the heavier Higgs boson masses depend already at tree-level on the soft SUSY-breaking parameters $m_{H_{1}}^{2}$ and $m_{H_{2}}^{2}$, and the $\mathrm{RG}$-evolution of the latter is very sensitive to the precise value of all of the other parameters, especially $h_{t}$. We also see that, as expected, the precise definition of the bottom Yukawa coupling has a sizeable effect on the Higgs boson masses only in the scenarios with large $\tan \beta$. Finally, we see that the choice of the renormalisation scheme for the values of the Higgs boson masses entering the one-loop corrections also induces a small variation in the final results for the physical Higgs boson masses. We note, however, that this variation amounts to a two-loop effect controlled by the electroweak gauge couplings, thus it is of higher order with respect of the accuracy of our calculation.

\subsection{Estimate of the theoretical uncertainties in the cMSSM}

In this subsection, we will discuss the shifts on the MSSM neutral Higgs boson masses induced by the choice of a different renormalisation scheme and by the variation of the renormalisation scale. We will also try to estimate the impact of the approximation of zero external momentum in the two-loop corrections. These shifts indicate the size of the higher order radiative corrections that are left uncomputed, and thus give a rough estimate of the theoretical uncertainties in the calculation of the masses.

\subsubsection{Comparing the $\overline{\mathrm{DR}}$ and OS calculations}

The first interesting comparison to make is between the results of our $\overline{\mathrm{DR}}$ calculation of the $\mathrm{CP}$-even Higgs boson masses and those of the OS calculation implemented in FeynHiggs. The latter is based on the one-loop formulae of Refs. [15, 32] and the two-loop formulae of Ref. [20] (for the $\mathcal{O}\left(\alpha_{t} \alpha_{s}\right)$ part) and Refs. [24, 25, 26] (for the $\mathcal{O}\left(\alpha_{b} \alpha_{s}\right), \mathcal{O}\left(\alpha_{t}^{2}\right)$ and $\mathcal{O}\left(\alpha_{t} \alpha_{b}+\alpha_{b}^{2}\right)$ parts, respectively). In the computation of FeynHiggs, the one-loop corrections are expressed in terms of physical masses and mixing angles, and appropriate counterterm contributions are inserted in the two-loop corrections for consistency.

As FeynHiggs does not perform the evolution of the MSSM parameters from the highenergy input scale to $M_{\mathrm{EWSB}}$, in order to obtain the Higgs boson masses in the SPS scenarios we have to provide it with the input parameters at the weak scale, as they are computed by one of the RGE codes. In particular, the top quark mass must be the physical one, $M_{t}$; the parameters $\mu$ and $\tan \beta$ must be the running ones, computed at the scale $M_{\mathrm{EWSB}}$; the squark masses and mixings can be either physical or running (in the latter case they are converted by FeynHiggs into the physical ones by applying the appropriate one-loop corrections); the definition of the gaugino mass parameters is irrelevant at the perturbative 
order we are interested in; finally, the $A$ boson mass, which enters the tree-level mass matrix of the CP-even Higgs bosons, must be the physical one, computed at the two-loop accuracy.

Table 9 shows the values of $M_{h}$ and $M_{H}$ as computed by FeynHiggs (version 1.5.1) in the six SPS scenarios, with the weak scale input parameters provided by SuSpect. The corresponding results from the pure SuSpect calculation are also shown for comparison. It can be seen from table 9 that the values of $M_{h}$ resulting from the OS calculation of FeynHiggs are consistently a couple of $\mathrm{GeV}$ larger than the ones provided by the $\overline{\mathrm{DR}}$ calculation. As the $\overline{\mathrm{DR}}$ and OS computations are equivalent up to the two-loop $\mathcal{O}\left(\alpha_{t} \alpha_{s}+\alpha_{b} \alpha_{s}+\alpha_{t}^{2}+\alpha_{t} \alpha_{b}+\alpha_{b}^{2}\right)$ accuracy, the discrepancies must be due to terms that are formally of higher order, i.e. two-loop terms controlled by the electroweak gauge couplings and three-loop terms, among which the most important are controlled by the top Yukawa and strong gauge couplings. Concerning the heavier Higgs boson mass, $M_{H}$, it can be seen in table 9 that the results of FeynHiggs are in excellent agreement with those of SuSpect. This fact is not surprising, because for $M_{A} \gg M_{Z}$, as is always the case in the SPS scenarios, $M_{H}$ and $M_{A}$ are very strictly correlated, and in our comparison FeynHiggs takes as input the value of $M_{A}$ as computed by SuSpect.

\begin{tabular}{|c|l|c|c|c|c|c|c|}
\hline Mass & Code & SPS1a & SPS2 & SPS4 & SPS5 & SPS8 & SPS9 \\
\hline \multirow{2}{*}{$M_{h}$} & SuSpect & 112.1 & 116.8 & 114.1 & 116.1 & 115.5 & 117.5 \\
& FeynHiggs & 113.8 & 118.3 & 116.1 & 118.5 & 117.3 & 118.3 \\
\hline \multirow{2}{*}{$M_{H}$} & SuSpect & 406.5 & 1552.1 & 355.3 & 686.9 & 550.6 & 1056.6 \\
& FeynHiggs & 406.5 & 1552.0 & 354.8 & 686.5 & 550.6 & 1056.7 \\
\hline
\end{tabular}

Table 9: The lighter and heavier CP-even Higgs boson masses, $M_{h}$ and $M_{H}$, in the six SPS scenarios, as computed by SuSpect and FeynHiggs. The weak scale input parameters, including the physical mass $M_{A}$, are taken from the output of SuSpect.

\subsubsection{Renormalisation scale dependence}

Another measure of the effect of the higher orders consists in studying the numerical dependence of the results for the physical Higgs masses on the renormalisation scale $M_{\mathrm{EwSB}}$ at which the effective potential is minimised and the radiatively corrected masses are computed. In the ideal case of an all-orders calculation, the physical observables should not depend on the choice of the scale. The residual scale dependence still present in the real case can be taken as a rough estimate of the magnitude of the corrections that are left uncomputed. 

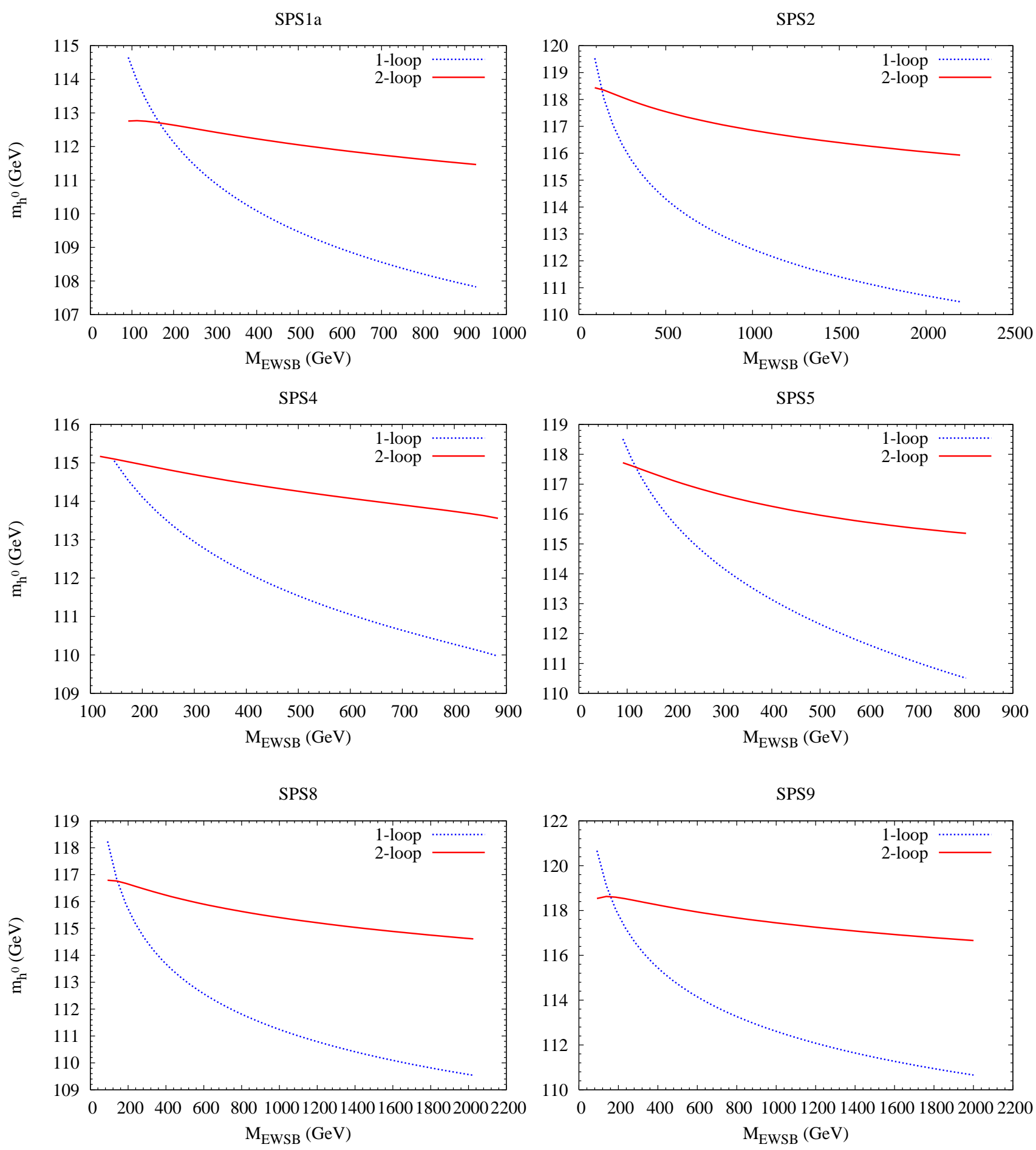

Figure 1: The lighter CP-even Higgs boson mass, $M_{h}$, as computed by SoftSusy in the six SPS scenarios, as a function of the minimisation scale of the effective potential, $M_{\text {EWSB }}$. The dotted lines correspond to the one-loop computation and the solid lines to the two-loop computation. 

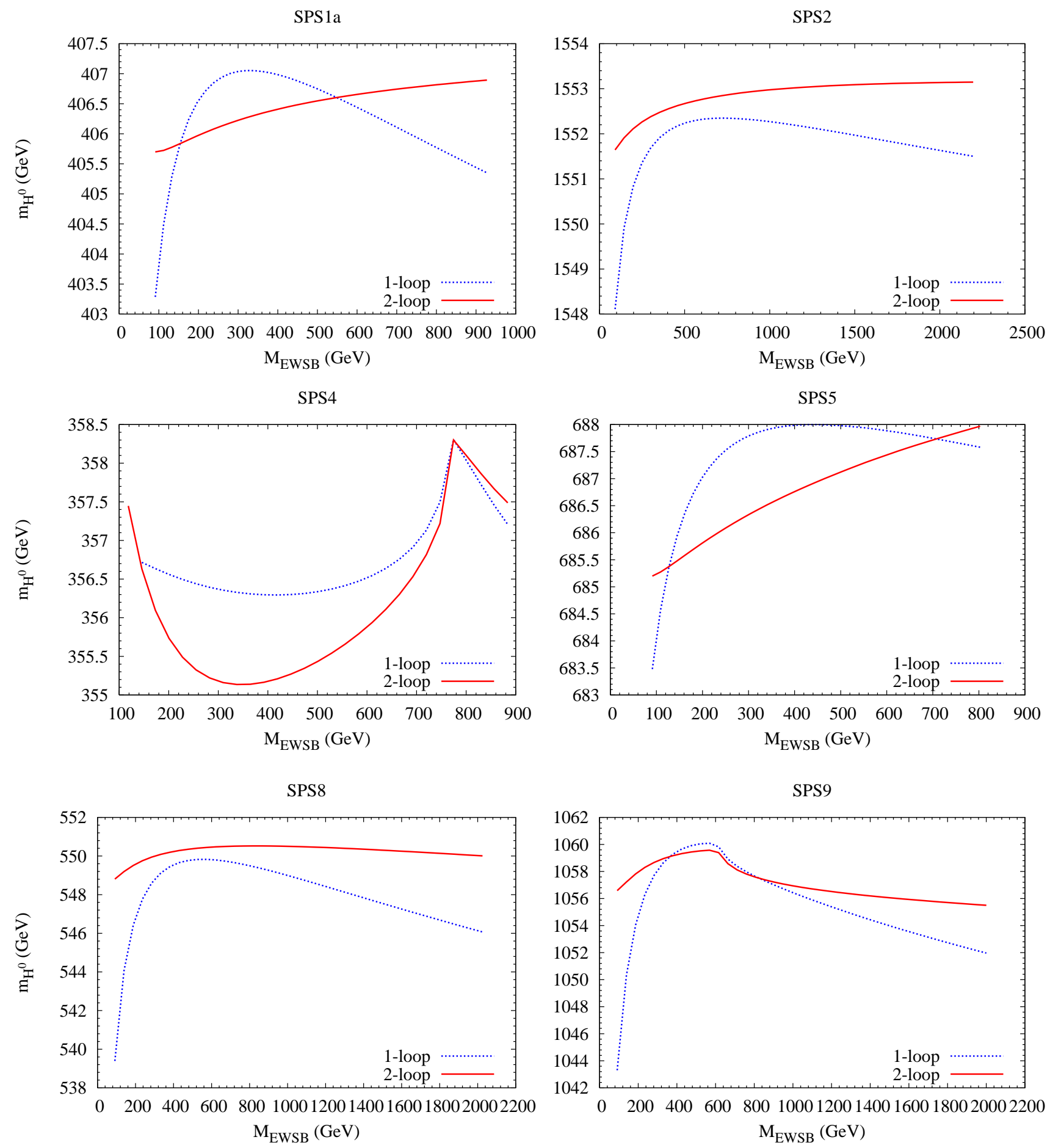

Figure 2: Same as figure 1 for the heavier $\mathrm{CP}$-even Higgs boson mass, $M_{H}$. 
Figs. 1 and 2 show the renormalisation scale dependence of the CP-even Higgs boson masses $M_{h}$ and $M_{H}$, respectively, as computed by SoftSusy in the six SPS scenarios discussed in section 3.1. The scale dependence of $M_{A}$ is very similar to that of $M_{H}$ and is thus omitted for brevity. We have also checked that the same features can be reproduced with SPheno and SuSpect. The dotted line in each plot corresponds to the one-loop computation of the relevant mass, whereas the solid line corresponds to the two-loop computation. The scale $M_{\mathrm{EWSB}}$ is varied from a minimum value of $M_{Z}$ to a maximum corresponding to twice the stop mass scale $\sqrt{m_{\tilde{t}_{1}} m_{\tilde{t}_{2}}}$.

It can be seen from fig. 1 that the one-loop results for the lighter Higgs boson mass $M_{h}$ show a sizeable scale dependence, varying by nearly $10 \mathrm{GeV}$ in the considered range of $M_{\mathrm{EWSB}}$. This dependence is essentially driven by the variation of the running top mass, which decreases as the renormalisation scale increases (we recall that the leading one-loop corrections to $M_{h}^{2}$ are proportional to $\left.m_{t}^{4} / v^{2}\right)$. On the other hand, it appears that the inclusion of the two-loop corrections significantly improves the scale dependence of $M_{h}$, compensating for the dependence on $m_{t}$ and leaving a residual variation of $2-3 \mathrm{GeV}$ in the considered range of $M_{\mathrm{EWSB}}$.

Fig. 2 shows that a similar situation occurs in the case of the heavier Higgs boson mass $M_{H}$, with the inclusion of the two-loop corrections clearly improving the renormalisation scale dependence of the results. One exception is given by the plot corresponding to the scenario SPS4, where the curves show a spike and the two-loop corrections do not improve the scale dependence. This anomalous behaviour is due to the fact that the codes use the running values of the Higgs boson masses in the computation of the one-loop corrections. For large values of $\tan \beta$ (e.g. $\tan \beta=50$ in SPS4) the running masses for the heavier Higgs bosons vary by hundreds of $\mathrm{GeV}$ as the renormalisation scale varies in the considered range. Thus, for some values of the renormalisation scale, the loop integrals involving the heavier Higgs boson masses encounter real-particle thresholds which give rise to the characteristic spike behaviour. We have checked that, if we use in the one-loop corrections the physical values of the Higgs boson masses, which vary only of a few $\mathrm{GeV}$ in the considered range of $M_{\mathrm{EWSB}}$, the spike disappears and the plot for the SPS4 scenario looks qualitatively very similar to the ones for the other scenarios (the same happens for another large-tan $\beta$ scenario, SPS1b, which we did not include in our analysis). We recall, however, that the choice of whether to use the running or physical Higgs masses in the one-loop corrections amounts to a higher-order effect with respect to the accuracy of our calculation.

\subsubsection{The external momentum dependence}

To simplify the calculation of the two-loop radiative corrections to the Higgs boson masses, the squared external momentum $p^{2}$ in the two-loop part of the self energies has been set to zero, rather than to the squared mass of the corresponding Higgs boson. This approximation can be considered as a source of theoretical uncertainty. The actual size of the shift on the Higgs boson masses induced by this approximation can only be obtained when the exact two- 
loop computation, retaining the external momentum dependence, is performed (see Ref. [43] for a first attempt). However, it can be roughly estimated by considering the error that the zero-momentum approximation induces in the one-loop corrections, and assuming that the error induced in the two-loop corrections will have the same relative size (a similar analysis was performed in Ref. [33]).

\begin{tabular}{|l|c|c|c|c|c|c|c|}
\hline Mass & Approximation & SPS1a & SPS2 & SPS4 & SPS5 & SPS8 & SPS9 \\
\hline$M_{h}$ & $p^{2}=0$ in 1-loop & +0.9 & +2.0 & +1.3 & +1.0 & +2.0 & +1.7 \\
& Estimated 2-loop effect & 0.1 & 0.4 & 0.2 & 0.1 & 0.3 & 0.3 \\
\hline \multirow{2}{*}{$M_{H}$} & $p^{2}=0$ in 1-loop & -0.3 & +0.8 & +4.4 & +0.02 & -0.1 & -0.9 \\
& Estimated 2-loop effect & 0.02 & 0.1 & 0.7 & 0.02 & $<0.01$ & 0.04 \\
\hline
\end{tabular}

Table 10: Effect of the zero-momentum approximation in the one-loop corrections to the $\mathrm{CP}$-even Higgs boson masses, $M_{h}$ and $M_{H}$, in the six SPS scenarios, and an estimate of the corresponding two-loop effect. All the numbers are in $\mathrm{GeV}$.

In table 10, we display the effect of the approximation of zero external momentum on the lighter and heavier CP-even Higgs boson masses, as computed by SuSpect in the six SPS scenarios. For each mass, the first row contains the shift (in $\mathrm{GeV}$ ) resulting from the approximation $p^{2}=0$ in the one-loop part of the corrections, and the second row contains the estimate for the size of the same effect in the two-loop case. The latter is obtained by scaling the absolute value of the shift induced in the one-loop corrections by the ratio between the size of the two-loop zero-momentum corrections and that of the one-loop zero-momentum corrections in the considered scenario. As can be seen, the effect of the zero-momentum approximation on the one-loop calculation of $M_{h}$ is of the order of $1-2 \mathrm{GeV}$. When rescaled to account for the relative size of the two-loop to the one-loop corrections, this results in a shift of less than half $\mathrm{GeV}$ in all of the considered scenarios.

On the other hand, we see from table 10 that in all scenarios but SPS4 the effect of the zero-momentum approximation on the one-loop computation of $M_{H}$ is of the order of a few tenths of $\mathrm{GeV}$. As a consequence, one can predict that in those scenarios the effect of the same approximation on the two-loop corrections is negligible. This is due to the fact that, as mentioned in section 3.2.2, the heavier Higgs boson mass is dominated by the tree-level value, and the impact of the one-loop radiative corrections is anyway small. In the case of the large- $\tan \beta$ scenario SPS4, however, there are non-negligible momentum-dependent contributions from the one-loop diagrams with bottom quarks and tau leptons, inducing a shift of more than $4 \mathrm{GeV}$ in $M_{H}$, and the effect of the zero-momentum approximation on the two-loop corrections can be predicted to get close to one $\mathrm{GeV}$. 


\subsection{The case of the pMSSM}

We now discuss the case of the phenomenological MSSM defined in section 3.1. In principle, since there are 22 free parameters in the model, the phenomenological analyses should be rather complicated to carry out. However, as mentioned previously, only a small subset of parameters plays a significant role in the evaluation of the Higgs boson masses. Before presenting our numerical results, we briefly summarise the role and the main effects of the various MSSM parameters.

At the tree level, the Higgs sector of the pMSSM can be described by two input parameters in addition to the SM ones; these parameters are in general taken to be the mass of the pseudoscalar Higgs boson $M_{A}$ and $\tan \beta$. In this parameterisation, the tree-level $\mathrm{CP}$-even Higgs boson masses are given by

$$
M_{h / H}=\frac{1}{\sqrt{2}}\left[M_{A}^{2}+M_{Z}^{2} \mp \sqrt{\left(M_{A}^{2}+M_{Z}^{2}\right)^{2}-4 \cos ^{2} 2 \beta M_{A}^{2} M_{Z}^{2}}\right]^{1 / 2} .
$$

The charged Higgs boson mass is given at tree-level by $M_{H^{ \pm}}=\sqrt{M_{A}^{2}+M_{W}^{2}}$, while the angle $\alpha$ in the $\mathrm{CP}$-even Higgs sector is given by $\tan 2 \alpha=\tan 2 \beta\left(M_{A}^{2}+M_{Z}^{2}\right) /\left(M_{A}^{2}-M_{Z}^{2}\right)$, with the constraint $-\frac{\pi}{2} \leq \alpha \leq 0$.

The maximal value of the lighter $\mathrm{CP}$-even Higgs boson mass at the tree level, $M_{h}=$ $\cos 2 \beta M_{Z}$, is obtained in the decoupling regime, $M_{A} \gg M_{Z}$ (note that, in this case, the lighter Higgs boson of the MSSM will have exactly the same properties as the SM Higgs boson), and is saturated for large values of $\tan \beta$. In this limit, the mass of the heavier $\mathrm{CP}-$ even Higgs boson is simply $M_{H}=M_{A}$.

The leading one-loop radiative corrections to $M_{h}^{2}$ are controlled by the top and bottom Yukawa couplings, and in the decoupling regime read

$$
\epsilon=\frac{3 m_{t}^{4}}{2 \pi^{2} v^{2}}\left(\ln \frac{M_{S}^{2}}{m_{t}^{2}}+\frac{X_{t}^{2}}{M_{S}^{2}}-\frac{X_{t}^{4}}{12 M_{S}^{4}}\right)-\frac{3 m_{b}^{4}}{2 \pi^{2} v^{2}} \frac{X_{b}^{4}}{12 M_{S}^{4}},
$$

where $M_{S}$ is a common soft SUSY-breaking mass term for the third-generation squarks and $X_{t, b}$ are given in terms of the stop/sbottom trilinear couplings, $\mu$ and $\tan \beta$, by:

$$
X_{t}=A_{t}-\mu \cot \beta \text { and } X_{b}=A_{b}-\mu \tan \beta \text {. }
$$

For $M_{A} \gg M_{Z}$ and $\tan \beta \gg 1$, one obtains $M_{h}^{2}=M_{Z}^{2}+\epsilon$ for the lighter $\mathrm{CP}$-even Higgs boson, while for the heavier Higgs boson one still has $M_{H}=M_{A}$. The choice of the renormalisation scheme for the parameters entering eq. (11) becomes relevant when the two-loop corrections are included; we recall that in our calculation we express the one-loop corrections in terms of $\overline{\mathrm{DR}}$ parameters.

Because of the quartic dependence on $m_{t}$, the corrections controlled by the top Yukawa coupling are the leading ones. These corrections are larger when the logarithm in the first term of eq. (11) is larger, i.e. for large $M_{S}$ values (corresponding to large stop masses). In 
addition, the top quark corrections are maximal in the so-called $M_{h}^{\max }$ scenario [45], where the trilinear stop coupling in the $\overline{\mathrm{DR}}$ scheme is such that $X_{t} \sim \sqrt{6} M_{S}$.

The corrections controlled by the bottom Yukawa coupling are in general strongly suppressed with respect to those controlled by the top Yukawa coupling, due to the overall factor $m_{b}^{4}$. However, in the last term of eq. (11), proportional to $X_{b}^{4}$, this suppression can be compensated by a large value of the product $\mu \tan \beta$, providing a non-negligible negative correction to $M_{h}^{2}$. The choice of the values for the remaining soft SUSY-breaking parameters does not have a very large impact on the one-loop corrections, and in the $\overline{\mathrm{DR}}$ calculation the two-loop corrections, although numerically significant in the determination of the precise value of the lighter Higgs boson mass, do not substantially alter the picture.

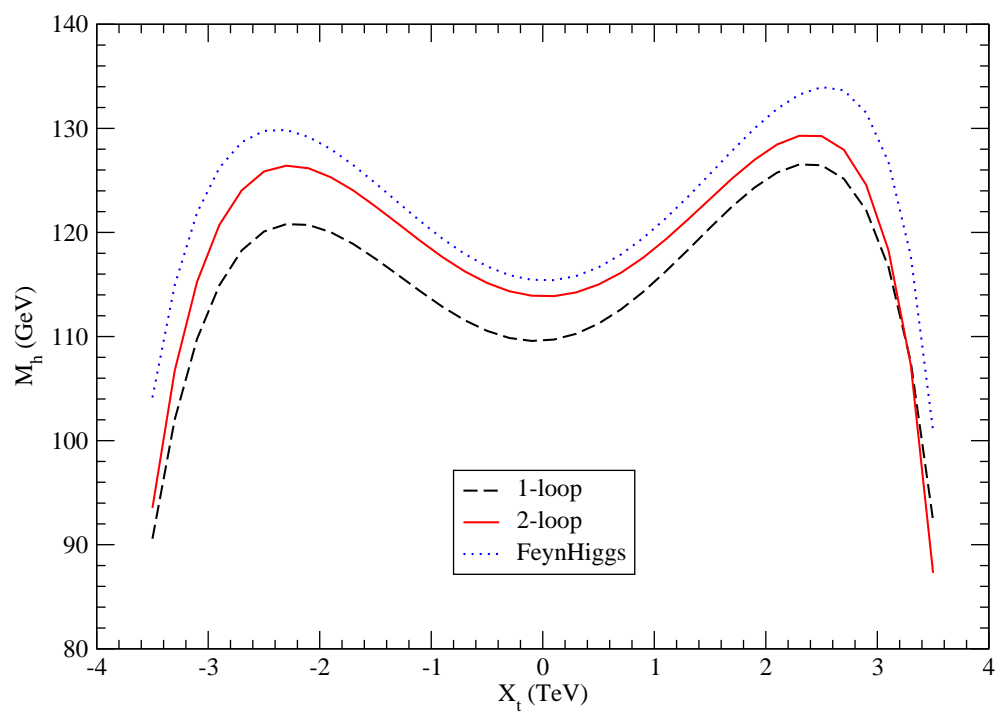

Figure 3: The lighter MSSM Higgs boson mass as a function of $X_{t}$ in the $\overline{\mathrm{DR}}$ scheme for $\tan \beta=10$ and $M_{S}=M_{A}=1 \mathrm{TeV}$ with $M_{t}=178 \mathrm{GeV}$. The full and dashed lines correspond, respectively, to the two-loop and one-loop corrected masses as calculated with SuSpect, while the dotted line corresponds to the two-loop $M_{h}$ value obtained with FeynHiggs.

The above features are exemplified in fig. 3, where the lighter Higgs boson mass is displayed as a function of the $\overline{\mathrm{DR}}$ parameter $X_{t}$, for $M_{t}=178$. In the figure, the MSSM parameters are set to those of the three pMSSM points introduced in section 3.1; in particular, the physical pseudoscalar mass $M_{A}$ and the third-generation soft SUSY-breaking scalar masses $M_{S}$ (the latter computed at the renormalisation scale $Q=1 \mathrm{TeV}$ ) are set to $1 \mathrm{TeV}$, while $\tan \beta$ is fixed to $\tan \beta=10$ at $Q=M_{Z}$. The dashed curve for the one-loop corrections, and the full curve for the two-loop corrections in the $\overline{\mathrm{DR}}$ scheme, have been obtained using the program SuSpect. As one can see, the lighter Higgs boson mass $M_{h}$ has a local minimum for zero stop mixing, and it increases with $\left|X_{t}\right|$ until it reaches a local maximum at the point $\left|X_{t}\right|=\sqrt{6} M_{S} \sim 2.45 \mathrm{TeV}$, where it starts to decrease again. Note 
that the two-loop corrections amount to several $\mathrm{GeV}$ and, in this scenario, decrease in size when $X_{t}$ takes on large and positive values.

The dotted curve in fig. 3 is obtained with the program FeynHiggs, using the MSSM input parameters as they come from SuSpect. In the OS renormalisation scheme adopted by FeynHiggs, the maximal $M_{h}$ value is obtained for $X_{t}^{\mathrm{OS}} \sim 2 M_{S}^{\mathrm{OS}}$, where $X_{t}^{\mathrm{OS}}$ and $M_{S}^{\mathrm{OS}}$ are the unphysical parameters obtained by rotating the diagonal matrix of the OS stop masses by the OS mixing angle (see e.g. Ref. [47] for a discussion). On the other hand, as we are plotting $M_{h}$ as a function of the $\overline{\mathrm{DR}}$ parameter $X_{t}$ for both the SuSpect and FeynHiggs computations, we find the maximum value of $M_{h}$ roughly at the same place. Comparing the solid and dotted lines, it can be seen that for small to moderate values of $X_{t}$ the results of the OS calculation of FeynHiggs are a couple of GeV higher than those of the $\overline{\mathrm{DR}}$ calculation, as in the constrained MSSM scenarios. Around the maxima of the curve, however, the difference between the two computations reaches $4-5 \mathrm{GeV}$. This indicates that for large mixing in the stop sector, $X_{t} \sim \sqrt{6} M_{S}$, the corrections which are formally of higher order (such as the three-loop terms controlled by the top Yukawa coupling and the strong coupling) are larger.

We can now discuss the numerical results that we obtain with some of the spectrum generators in the three pMSSM scenarios defined in section 3.1. Table 11 shows the results obtained with the spectrum generators SPheno and SuSpect for the masses of the lighter and heavier CP-even Higgs bosons in the three pMSSM scenarios (the option for the pMSSM mode is not yet completely implemented in SoftSusy). It can be seen from the table that the results of the two codes agree within less than a $\mathrm{GeV}$ for $M_{h}$, as was the case in the constrained MSSM scenarios (see table 1). The residual discrepancies are due, as discussed in section 2, to the different ways in which the two codes implement the various threshold corrections to gauge and Yukawa couplings at the scale $Q=M_{Z} 2$. On the other hand, it can be seen that the two codes are in excellent agreement for the heavier CP-even Higgs boson mass. This is due to the strict correlation between $M_{H}$ and $M_{A}$ : contrary to the case of the constrained MSSM scenarios, where $M_{A}$ is determined through the EWSB conditions and is thus sensitive to all of the details of the RG procedure, in the pMSSM scenario $M_{A}$ is given as an input and is thus the same for both codes.

The impact of the radiative corrections on the Higgs boson mass calculations is similar to what has been discussed in the case of the constrained scenarios and will not be repeated here. Furthermore, for the heavier CP-even Higgs boson, all of the theoretical uncertainties discussed in section 3.3 are very small, generating shifts of less than $100 \mathrm{MeV}$. In the following, we will therefore only concentrate on the uncertainties in the evaluation of the lighter Higgs boson mass $M_{h}$ due to the renormalisation scheme, the renormalisation scale and the dependence on the external momentum. The results, obtained using SuSpect, are summarised in table 12 for the three pMSSM points.

\footnotetext{
${ }^{2} \mathrm{~A}$ further difference between the codes is that, in the pMSSM mode, SuSpect uses the physical Higgs boson masses in the computation of the various threshold corrections at $Q=M_{Z}$, whereas SPheno switches to the physical masses only if one of the squared running masses becomes negative at $Q=M_{Z}$.
} 


\begin{tabular}{|l|c|ccc|}
\hline Mass & Code & pMSSM1 & pMSSM2 & pMSSM3 \\
\hline$M_{h}$ & SPheno & 114.3 & 118.8 & 130.0 \\
& SuSpect & 113.8 & 118.4 & 129.4 \\
\hline$M_{H}$ & SPheno & 1000.2 & 1000.1 & 999.4 \\
& SuSpect & 1000.2 & 1000.2 & 999.5 \\
\hline
\end{tabular}

Table 11: The lighter and heavier CP-even Higgs boson masses, $M_{h}$ and $M_{H}$, in the pMSSM scenarios as computed by SPheno2.2.1 and SuSpect2.3. The SM and MSSM input parameters are chosen as in section 3.1 .

\begin{tabular}{|c|c|c|c|}
\hline Shift & pMSSM1 & pMSSM2 & pMSSM3 \\
\hline Default value & 113.8 & 118.4 & 129.4 \\
\hline \hline FeynHiggs shift & +1.6 & +1.9 & +4.4 \\
\hline$M_{\text {EWSB }}=150 \mathrm{GeV}$ & +1.1 & +1.5 & +4.4 \\
$M_{\mathrm{EWSB}}=2 \mathrm{TeV}$ & -0.7 & -0.8 & -0.8 \\
\hline$p^{2}=0$ in 1-loop & +1.6 & +1.8 & +3.0 \\
Estimated 2-loop effect & +0.3 & +0.2 & +0.2 \\
\hline
\end{tabular}

Table 12: Effect of renormalisation scheme, renormalisation scale and estimate of the external momentum dependence in the determination of $M_{h}$ for the three pMSSM scenarios (all the shifts are in $\mathrm{GeV}$ ).

The first line of the table, after the default values given by SuSpect, shows the shift in the value of $M_{h}$ when it is calculated in the OS scheme by the program FeynHiggs, where the weak scale input parameters are taken from SuSpect. As mentioned previously, the difference between the two calculations of $M_{h}$ amounts to a couple of GeV in the first two scenarios, but reaches $4.4 \mathrm{GeV}$ in the large-mixing scenario pMSSM3.

To estimate the renormalisation scale dependence of the Higgs boson masses in the pMSSM scenarios, we can vary the scale $M_{\mathrm{EWSB}}$ at which the corrections are computed. By default, in the pMSSM scenarios this scale is identified with the scale at which the input parameters are given. In order to estimate the related uncertainty, we evolve the parameters of the three pMSSM scenarios from the input scale $Q=1 \mathrm{TeV}$ to some new scale, and compute again the Higgs boson masses in terms of the new values of the parameters (there is an option in SuSpect allowing for this study). The resulting shifts in the lighter Higgs boson mass for two alternative values of the scale, $M_{\mathrm{EWSB}}=150 \mathrm{GeV}$ and $M_{\mathrm{EWSB}}=2 \mathrm{TeV}$, are shown in the middle part of table 12, As can be seen, the shifts in $M_{h}$ are usually of the order of $1 \mathrm{GeV}$, but again reach $4.4 \mathrm{GeV}$ in the case of the pMSSM3 scenario. 
Finally, we see from the lower part of table 12 that the external momentum dependence of $M_{h}$ at the two-loop level, as estimated from the one-loop momentum dependence calculated in SuSpect, is expected to generate a shift of $300 \mathrm{MeV}$ or less, as was approximately the case in the cMSSM scenarios discussed in the previous sections.

\subsection{Summary of the theoretical uncertainties}

From the results presented in the previous sections, it is possible to attempt an estimate of the theoretical uncertainty that still affects the calculation of the lighter MSSM Higgs boson mass. Such an estimate is relevant because it affects the information on the MSSM parameter space that can be extracted from the comparison between the theoretical prediction on $M_{h}$ and the present experimental lower bounds. The phenomenological implications of this theoretical uncertainty will be further discussed in section 4 .

It has been shown in sections 3.3 .1 and 3.4 that the discrepancies between the OS and $\overline{\mathrm{DR}}$ calculations of the lighter Higgs boson mass $M_{h}$, which formally amount to a combination of two-loop effects controlled by the electroweak gauge couplings and three-loop effects, are of the order of 2-3 GeV in most of the considered cMSSM and pMSSM scenarios, but they can reach $4-5 \mathrm{GeV}$ in the pMSSM scenario with large stop mixing. Another measure of the size of the uncomputed corrections is given by the residual renormalisation scale dependence, and in sections 3.3 .2 and 3.4 it has been shown that the results of the $\overline{\mathrm{DR}}$ calculation of $M_{h}$ vary by $2-3 \mathrm{GeV}$ when the renormalisation scale is varied in a reasonable range. The discrepancies between the results of the different spectrum generators in the constrained MSSM scenarios discussed in section 3.2.1 correspond generally to three-loop effects (i.e., two-loop differences in the determination of parameters that enter the Higgs mass corrections at one loop), and in the case of $M_{h}$ are contained within a half GeV. Finally, another source of theoretical uncertainty is the approximation of zero external momentum employed in the two-loop corrections. In sections 3.3.3 and 3.4 it has been shown that this uncertainty in $M_{h}$ is less than half $\mathrm{GeV}$ in the considered scenarios.

The way these different results should be combined into a figure for the residual theoretical uncertainty on $M_{h}$ is debatable, but we think that a reasonably conservative estimate of the global uncertainty can be taken as $3-5 \mathrm{GeV}$. The lower value is expected in most of the cMSSM and pMSSM scenarios, while the upper value is expected in some special scenarios such as in the pMSSM with large mixing in the stop sector.

In Ref. [33] the combined effect of the corrections yet to be computed was estimated to induce an uncertainty in $M_{h}$ of the order of $3 \mathrm{GeV}$. We find that our results are in good agreement with that earlier estimate for most of the scenarios that we have discussed in this paper. A recent analysis with the program FeynHiggs [48], gives for the uncertainty on $M_{h}$ a range that is similar to the one that we quote here, i.e. $\Delta M_{h} \sim 3-5 \mathrm{GeV}$, depending on the considered MSSM scenario.

The fact that the predicted experimental accuracy in the determination of $M_{h}$ at a future linear collider is $50 \mathrm{MeV}$ [4] indicates that a huge effort is still necessary in order to improve 
the theoretical uncertainty on the Higgs boson masses up to the level required to compare with the forthcoming experimental results. An experimental effort is also needed to measure the SM input parameters more precisely, since they generate an error on $M_{h}$ which is of the same order as the theoretical uncertainty, as will be discussed now.

\subsection{The impact of the experimental uncertainties}

It is important to discuss how the predictions for the MSSM Higgs boson masses are affected by the experimental errors in the determination of the input SM parameters. This is a source of uncertainty independent of the ones discussed above, and it can be reduced by more precise experimental information.

It is well known, for example, that the precise values of the Higgs boson masses in the MSSM depend strongly on the exact value of the input top quark mass. Indeed, because the dominant one-loop corrections to the Higgs masses grow as $M_{t}^{4}$, a shift of several $\mathrm{GeV}$ on $M_{t}$ will produce a significant variation of $M_{h}$. Since the experimental error on the top quark pole mass, $\Delta M_{t}= \pm 4.3 \mathrm{GeV}$, is rather large, it will have a large impact on the numerical values of the Higgs masses. The benefits arising from a more precise measurement of $M_{t}$ for the determination of the MSSM parameters have been discussed in Ref. [50].

Tables 13 and 14 show the variation of $M_{h}$ and $M_{H}$, respectively, in the six SPS scenarios, resulting from a $\pm 1 \sigma$ variation in the top quark mass $M_{t}$ and in other SM input parameters, i.e. the bottom quark mass $m_{b}\left(m_{b}\right)^{\overline{\mathrm{MS}}}$, and the strong and electromagnetic coupling constants $\alpha_{s}\left(M_{Z}\right)^{\overline{\mathrm{MS}}}$ and $\alpha_{\mathrm{em}}^{-1}\left(M_{Z}\right)^{\overline{\mathrm{MS}}}$, which are given in eqs. (8) -(9); the other SM input parameters, such as $G_{F}, M_{Z}$ and $M_{\tau}$, are so precisely measured that the impact of their experimental errors on the Higgs boson masses is negligible.

As expected, the effect of the error on the top quark mass is rather large. In the case of the lighter $\mathrm{CP}$-even Higgs boson, a $4.3 \mathrm{GeV}$ variation in $M_{t}$ results in a shift in $M_{h}$ of approximately $2 \mathrm{GeV}$ in most SPS scenarios 3 with larger (lower) values of $M_{t}$ increasing (decreasing) the obtained value for $M_{h}$; an exception is given by the scenario SPS5, where, due to a large stop mixing and a low $\tan \beta$ value, the top quark mass plays a more prominent role, and the shift can reach $3 \mathrm{GeV}$. In the case of the heavier $\mathrm{CP}$-even Higgs boson, the variations are at the level of a few percent, except for the SPS4 scenario, where they reach the level of $10 \%$. Differently from the case of the lighter Higgs boson mass, $M_{H}$ can either decrease or increase with increasing (or decreasing) $M_{t}$. This is due to the fact that, as discussed previously, the heavier Higgs boson masses are mostly sensitive to the values of the soft SUSY-breaking Higgs mass parameters, whose RGE evolution has a complicated dependence on the top Yukawa coupling.

The impact on the value of $M_{h}$ of the experimental error on the bottom quark mass, $m_{b}\left(m_{b}\right)^{\overline{\mathrm{MS}}}=4.25 \pm 0.25 \mathrm{GeV}$, is significant only in the SPS4 scenario with high tan $\beta$, where

\footnotetext{
${ }^{3}$ There is a well known rule of thumb for the so-called $M_{h}^{\max }$ scenario, according to which a variation of one $\mathrm{GeV}$ in $M_{t}$ results into a variation of one $\mathrm{GeV}$ in $M_{h}$. We notice that this rule does not generally hold in the constrained MSSM scenarios.
} 
it can lead to a shift of about $100 \mathrm{MeV}$; otherwise, it is negligible. The effect, however, can reach the level of $10 \%$ or so for the determination of $M_{H}$ in this high $\tan \beta$ scenario, since, in this case, the bottom Yukawa coupling plays an important role in the evolution of the soft SUSY-breaking Higgs parameters upon which $M_{H}$ depends. Note that lower (higher) values of the bottom quark mass in general increase (decrease) the values of $M_{H}$.

The main effect of the error on the strong coupling constant is to generate an uncertainty in the top and bottom quark running masses, which propagates on the soft SUSY-breaking parameters and eventually on the Higgs mass spectrum. Varying $\alpha_{s}\left(M_{Z}\right)^{\overline{\mathrm{MS}}}=0.1172 \pm 0.002$ within its $1 \sigma$ range leads in general to an uncertainty of less than one per mille for $M_{h}$ and one percent for $M_{H}$ (an exception being the scenario SPS4 where the uncertainty for $M_{H}$ is two percent).

Finally, as one might have expected, the uncertainty arising from the error on the electromagnetic fine structure constant, $\alpha^{-1}\left(M_{Z}\right)^{\overline{\mathrm{MS}}}=127.934 \pm 0.027$ (due to the hadronic contribution uncertainties), is essentially negligible: in all cases, it barely reaches the per mille level for both $M_{h}$ and $M_{H}$.

We now turn to the case of the pMSSM. First of all, in the considered scenarios the errors due to the variation of all SM parameters within their $1 \sigma$ range are completely negligible in the case of the heavier CP-even Higgs boson: the maximal shift is less than $100 \mathrm{MeV}$ (i.e. $\lesssim 0.01 \%$ ) and occurs when the top mass is varied within its $4.3 \mathrm{GeV}$ uncertainty. As discussed previously, this is due to the fact that in the pMSSM the value $M_{H}$ is essentially controlled by the one of $M_{A}$, which is fixed to $1 \mathrm{TeV}$ in these scenarios. Second, even in the case of the lighter Higgs boson, the errors due to the variation of $\alpha$ and $m_{b}$ are very small and can be safely neglected (the insensitivity to the precise value of $m_{b}$ is due to the moderate value chosen for $\tan \beta$ in the considered scenarios).

The only SM parameters which have an impact on the value of $M_{h}$ in the considered scenarios are $M_{t}$ and $\alpha_{s}$. The shifts due to their experimental errors in the three scenarios are shown in table 15. The main effect of the variation of $\alpha_{s}$ is to shift the $\overline{\mathrm{DR}}$ top mass $m_{t}$ which enters the loop corrections to the lighter Higgs boson mass; the shift in $M_{h}$ increases with the magnitude of the stop mixing and reaches the level of $300 \mathrm{MeV}$ in the large mixing scenario. The largest uncertainty is, as expected, due to the experimental error on the top quark mass. The effect follows the same trend as in the SPS scenarios: for small mixing (pMSSM1) the induced error is of about $2 \mathrm{GeV}$, and it reaches the level of $4 \mathrm{GeV}$ in the large mixing scenario (pMSSM3). To obtain an accuracy of $50 \mathrm{MeV}$ on the lighter Higgs boson mass (as will be measured experimentally at the future colliders) one needs an experimental determination of $M_{t}$ to better than $100 \mathrm{MeV}$. Our results are in full agreement with Ref. [50], where the uncertainty in $M_{h}$ induced by the errors on $M_{t}, \alpha_{s}, m_{b}$ and $M_{W}$ is discussed.

In conclusion, the experimental uncertainties on the SM parameters lead to an error of about 3-4 GeV on the lighter Higgs boson mass $M_{h}$. This error is thus of the same magnitude as the total theoretical uncertainty which has been discussed in the previous section, but will be decreased when new measurements of the top mass become available. 


\begin{tabular}{|c|c|c|c|c|c|c|}
\hline Approximation & SPS1a & SPS2 & SPS4 & SPS5 & SPS8 & SPS9 \\
\hline Default & 112.1 & 116.8 & 114.1 & 116.2 & 115.4 & 117.5 \\
\hline$\Delta M_{t}= \pm 4.3 \mathrm{GeV}$ & $\begin{array}{l}+1.59 \\
-1.55\end{array}$ & $\begin{array}{l}+2.12 \\
-2.04\end{array}$ & $\begin{array}{l}+1.73 \\
-1.69\end{array}$ & $\begin{array}{l}+2.09 \\
-3.05\end{array}$ & $\begin{array}{l}+2.26 \\
-2.14\end{array}$ & $\begin{array}{l}+1.89 \\
-1.84\end{array}$ \\
\hline$\Delta m_{b}= \pm 0.25 \mathrm{GeV}$ & $\begin{array}{l}<0.01 \\
+0.02\end{array}$ & $\begin{array}{l}<0.01 \\
<0.01\end{array}$ & $\begin{array}{l}-0.07 \\
+0.09\end{array}$ & $\begin{array}{l}<0.01 \\
<0.01\end{array}$ & $\begin{array}{l}<0.01 \\
<0.01\end{array}$ & $\begin{array}{l}-0.01 \\
+0.01\end{array}$ \\
\hline$\Delta \alpha_{s}= \pm 0.002$ & $\begin{array}{l}+0.09 \\
-0.06\end{array}$ & $\begin{array}{l}-0.06 \\
+0.07\end{array}$ & $\begin{array}{l}+0.06 \\
-0.05\end{array}$ & $\begin{array}{l}-0.09 \\
+0.03\end{array}$ & $\begin{array}{l}-0.05 \\
+0.05\end{array}$ & $\begin{array}{l}+0.09 \\
{ }_{-0.10}\end{array}$ \\
\hline$\Delta \alpha^{-1}= \pm 0.027$ & $\begin{array}{l}+0.02 \\
<0.01\end{array}$ & $\begin{array}{l}+0.01 \\
<0.01\end{array}$ & $\underset{<0.01}{+0.01}$ & $\begin{array}{l}-0.01 \\
<0.01\end{array}$ & $\begin{array}{l}<0.01 \\
<0.01\end{array}$ & $\begin{array}{l}-0.01 \\
<0.01\end{array}$ \\
\hline
\end{tabular}

Table 13: The lighter CP-even Higgs boson mass, $M_{h}$, in the six SPS scenarios, as computed by SuSpect using different input values for the SM parameters. The first row contains the default values in $\mathrm{GeV}$, the other rows contain the shifts (in $\mathrm{GeV}$ ) due to the different inputs.

\begin{tabular}{|c|c|c|c|c|c|c|}
\hline Approximation & SPS1a & SPS2 & SPS4 & SPS5 & SPS8 & SPS9 \\
\hline Default & 405.9 & 1551.2 & 354.4 & 686.6 & 550.3 & 1056.6 \\
\hline$\Delta M_{t}= \pm 4.3 \mathrm{GeV}$ & $\begin{array}{l}+6.5 \\
-6.7\end{array}$ & ${ }_{-42.5}^{+42.6}$ & $\begin{array}{l}+27.2 \\
-29.4\end{array}$ & $\begin{array}{l}-11.1 \\
+7.5\end{array}$ & $\begin{array}{l}+16.0 \\
-15.8\end{array}$ & $\begin{array}{l}-15.6 \\
+10.2\end{array}$ \\
\hline$\Delta m_{b}= \pm 0.25 \mathrm{GeV}$ & $\begin{array}{l}-1.2 \\
+1.2\end{array}$ & $\begin{array}{l}-3.0 \\
+2.8\end{array}$ & $\begin{array}{l}-39.5 \\
+34.2\end{array}$ & $\begin{array}{l}-0.8 \\
+0.7\end{array}$ & $\begin{array}{l}-2.5 \\
+2.3\end{array}$ & $\begin{array}{l}-5.3 \\
+4.9\end{array}$ \\
\hline$\Delta \alpha_{s}= \pm 0.002$ & $\begin{array}{l}+2.2 \\
-1.9\end{array}$ & $\begin{array}{l}-7.1 \\
+7.4\end{array}$ & $\begin{array}{l}+6.3 \\
-6.2\end{array}$ & ${ }_{-6.1}^{+5.7}$ & $\begin{array}{l}+3.4 \\
-3.5\end{array}$ & $\begin{array}{l}+13.9 \\
-14.4\end{array}$ \\
\hline$\Delta \alpha^{-1}= \pm 0.027$ & $\begin{array}{l}+0.3 \\
-0.2\end{array}$ & $\begin{array}{l}+0.4 \\
-0.2\end{array}$ & $\begin{array}{l}+0.2 \\
-0.1\end{array}$ & $\begin{array}{l}+0.2 \\
-0.2\end{array}$ & $\begin{array}{l}-0.1 \\
+0.1\end{array}$ & $\begin{array}{l}-0.1 \\
<0.1\end{array}$ \\
\hline
\end{tabular}

Table 14: Same as table 13 for the heavier CP-even Higgs boson mass, $M_{H}$.

\begin{tabular}{|l|c|c|c|}
\hline Approximation & pMSSM1 & pMSSM2 & pMSSM3 \\
\hline Default & 113.83 & 118.39 & 129.35 \\
\hline$\Delta M_{t}= \pm 4.3 \mathrm{GeV}$ & ${ }_{-2.21}^{+2.31}$ & ${ }_{-2.60}^{+2.72}$ & ${ }_{-3.66}^{+3.83}$ \\
$\Delta \alpha_{s}= \pm 0.002$ & $\mp 0.17$ & $\mp 0.21$ & $\mp 0.30$ \\
\hline
\end{tabular}

Table 15: The lighter $\mathrm{CP}$-even Higgs boson mass, $M_{h}$, in the three pMSSM scenarios, as computed by SuSpect using different input values for the SM parameters. The first row contains the default values in $\mathrm{GeV}$, the other rows contain the shifts (in $\mathrm{GeV}$ ) due to the different $M_{t}$ and $\alpha_{s}$ inputs; the errors due to $\Delta m_{b}$ and $\Delta \alpha$ are negligible. 
Another independent source of uncertainty are the experimental errors in the determination of the masses and couplings of the supersymmetric particles. A detailed study of this issue goes beyond the scope of our paper.

\section{Phenomenological consequences}

In this section, we discuss the phenomenological impact of the radiative corrections in the MSSM Higgs sector and of the related theoretical and experimental uncertainties. In particular, we study the maximal values for the lighter Higgs mass as well as the constraints on $\tan \beta$ coming from the negative searches of the Higgs bosons at LEP2. The analyses will be performed in the context of the constrained MSSM models (mSUGRA, AMSB and GMSB), but also in the context of the unconstrained model (pMSSM).

\subsection{The upper bound on the lighter Higgs boson mass}

At the tree-level, the mass of the lighter MSSM Higgs boson $M_{h}$ is bounded from above by $M_{Z}$. Loop corrections increase this bound to $\sim 135 \mathrm{GeV}$ as discussed previously. The knowledge of the precise value of this upper bound is crucial for several reasons.

- It is entirely conceivable that the lighter Higgs boson be discovered before the SUSY particles. In this case, it is important to accurately know the maximal possible value of $M_{h}$ in order to discriminate between the SM and the MSSM. Furthermore, even if SUSY particles have been observed, the knowledge of this value, in the absence of extra information, could allow one to distinguish between the MSSM and some non minimal SUSY models where the Higgs sector is extended.

- Even in the context of the MSSM itself, the knowledge of the maximal $M_{h}$ value could allow one to discriminate between various scenarios of SUSY breaking. This approach would be then complementary to the study of the differences in the SUSY particle spectra induced by the various breaking schemes.

- The value $M_{h} \sim 135 \mathrm{GeV}$ has a rather special status in the decoupling regime $M_{A} \gg$ $M_{Z}$ (which occurs very often in the constrained scenarios) where all of the MSSM Higgs bosons except for the lighter are too heavy to be observed experimentally at the next generation of high-energy colliders. Indeed, if $M_{h}$ is below this critical value, the Higgs particle will dominantly decay into $b$ quark pairs, while above this value it will dominantly decay into $W$ bosons (for a detailed discussion of the decay modes, see Ref. [51] for instance).

\subsubsection{The upper bound on $M_{h}$ in the pMSSM}

As discussed in section 3.4, in the pMSSM only a small subset of input parameters have a significant impact on the MSSM Higgs sector. In first approximation, to obtain the maximal value of lighter Higgs boson mass one does not need to scan over the 22 free pMSSM 
parameters, but, rather, to choose the relevant parameters in such a way that the one-loop radiative correction $\epsilon$ in eq. (11) is maximised. In particular, one can obtain a reasonable approximation of the maximal $M_{h}$ when one has:

i) large values of the parameter $\tan \beta, \tan \beta \gtrsim 20$ (but still $\tan \beta \lesssim 60$ to keep the bottom Yukawa coupling in a perturbative regime);

ii) a decoupling regime with a heavy pseudoscalar Higgs boson, $M_{A} \sim \mathcal{O}(\mathrm{TeV})$;

iii) heavy stops, i.e. large $M_{S}$ values; we note, however, that heavier stops correspond to more fine tuning of the parameters in order to achieve the correct minimum of the Higgs potential [52] and we choose $M_{S}=2 \mathrm{TeV}$ as a maximal value;

iv) a stop trilinear coupling such that $X_{t}$ is close to $+\sqrt{6} M_{S}$, as exemplified by fig. 3 .

As a starting point, we realise the assumptions above by adopting the $M_{h}^{\max }$ scenario of Ref. [45], which was used as a benchmark point for the LEP2 Higgs analyses; we choose however to be conservative, scaling the relevant soft SUSY-breaking parameters by a factor of two and using the upper limit $\tan \beta \sim 60$ :

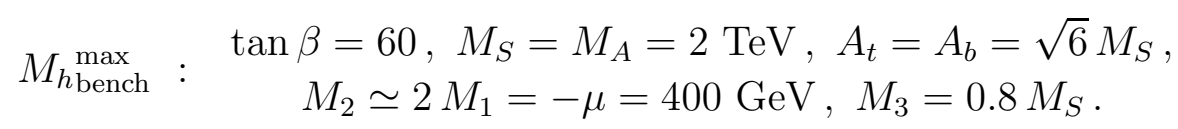

For the central value $M_{t}=178 \mathrm{GeV}$, and varying the values of $\alpha_{s}$ and $m_{b}$ (recall that we are in the large $\tan \beta$ regime where the bottom Yukawa coupling is enhanced) in their $1 \sigma$ allowed range, one obtains the maximal value of the lighter MSSM Higgs boson,

$$
M_{h \text { bench }}^{\max } \simeq 138 \mathrm{GeV} \text { for } M_{t}=178 \mathrm{GeV} .
$$

This value is higher than the one which is often quoted in the literature, $M_{h} \lesssim 135 \mathrm{GeV}$. The tendency of our $\overline{\mathrm{DR}}$ calculation to give lower values of $M_{h}$ than the OS calculation (using the same parameters as in Ref. [45] we would find $M_{h} \lesssim 130 \mathrm{GeV}$ ) is compensated by the increase of the central value of the top quark mass and by the fact that we conservatively set the SUSY-breaking scale to $2 \mathrm{TeV}$.

However, the bound in eq. (14) on the lighter Higgs boson mass is not yet fully optimised. In order to find the maximum maximorum $4 M_{h}$ value, one can still vary in a reasonable range the SUSY parameters entering the radiative corrections and add the estimated theoretical (and experimental) uncertainties. We have therefore studied how to tune the pMSSM parameters in such a way that the corrections to $M_{h}$ are maximised.

For instance, the one-loop electroweak corrections involving Higgs, gauge bosons and their fermionic superpartners tend to lower the value of $M_{h}$ when the chargino and neutralino masses are large. On the other hand, those corrections get small, allowing for a maximal $M_{h}$, when the chargino and neutralino masses are of the same order of the gauge boson masses (see e.g. Ref. [18] for a discussion). For $\mu \sim M_{2} \sim \frac{3}{2} M_{1} \sim 150 \mathrm{GeV}$, the upper bound on $M_{h}$

\footnotetext{
${ }^{4}$ We thank Daniel Treille for suggesting this expression.
} 
can be pushed upwards by about $2 \mathrm{GeV}$, while the lightest chargino mass is still larger than $104 \mathrm{GeV}$, as required by the negative searches at the LEP2 [46].

The gluino mass enters the two-loop QCD corrections in the Higgs sector and can have a non-negligible impact on the value of $M_{h}$. For large $\tan \beta$, lowering the value of the gluino mass parameter to $M_{3} \sim 0.4 M_{S}$ leads to an increase of $M_{h}$ of about $2 \mathrm{GeV}$.

Another increase of the upper bound on $M_{h}$ can be obtained by relaxing the equality of the stop mass parameters at the weak scale (this turns out to affect $M_{h}$ through changing the threshold correction to the top Yukawa coupling). For large $\tan \beta$, assuming $M_{\tilde{t}_{L}} \simeq$ $2 M_{\tilde{t}_{R}} \simeq 2.5 \mathrm{TeV}$ (which still keeps the EWSB scale, defined as the geometric mean of the two stop masses, in the vicinity of $2 \mathrm{TeV}$ ), one obtains an increase of $M_{h}$ of about $2 \mathrm{GeV}$.

One can then optimise the soft SUSY-breaking parameters to maximise all the effects mentioned above. Using the codes SPheno and SuSpect, we have performed a scan of the MSSM parameter space with the following constraints: $(i) M_{\text {EWSB }} \leq 2 \mathrm{TeV}$, even if some SUSY particles (in particular the heavier stop) may have masses above this value; (ii) the trilinear couplings of the third generation sfermions should not exceed the value $A_{t, b, \tau} \sim 3 M_{\mathrm{EWSB}}$, from which problems with charge and colour breaking (CCB) minima might appear [53]; (iii) all sparticles, in particular the lightest chargino and the gluino, should have masses that exceed their experimental bounds; (iv) the resulting spectrum should lead to acceptable contributions to the $\rho$ parameter, to the anomalous magnetic moment of the muon $(g-2)_{\mu}$ and to the radiative $b \rightarrow s \gamma$ decay 5 . The two codes give rather close results, the differences being of the order of $1 \mathrm{GeV}$. To provide a conservative upper bound on $M_{h}$, we quote the result of the code that gives the largest output value.

Using the central value of the top quark mass, we find the following upper bound on the lighter Higgs boson mass in the unconstrained MSSM:

$$
M_{h} \lesssim 143 \mathrm{GeV} \text { for } M_{t}=178 \mathrm{GeV} .
$$

Thus, tuning the SUSY parameters 6 in the way discussed above is very important, leading to an increase of $\sim 5 \mathrm{GeV}$ of the upper bound on $M_{h}$.

If we vary the top quark mass within its $1 \sigma$ experimental range we obtain the following upper bounds on $M_{h}$ for the lower and upper $M_{t}$ values

$$
\begin{aligned}
& M_{h} \lesssim 138 \mathrm{GeV} \text { for } M_{t}=173.7 \mathrm{GeV}, \\
& M_{h} \lesssim 148 \mathrm{GeV} \text { for } M_{t}=182.3 \mathrm{GeV} .
\end{aligned}
$$

Finally, to obtain the most conservative maximal value of $M_{h}$, one has to include the theoretical uncertainty. For the highest value of the top quark mass, $M_{t}=182.3 \mathrm{GeV}$, when

\footnotetext{
${ }^{5}$ The $(g-2)_{\mu}$ bound is easily satisfied as the second-generation sfermion masses can be taken to be large without affecting the Higgs mass calculation, while the $\Delta \rho$ constraint is in general weaker than the related CCB constraint. On the other hand, constraints coming from dark matter are not studied, being beyond the scope of this paper.

${ }^{6}$ For files detailing the choices of parameters that lead to the maximal $M_{h}$ and the resulting MSSM mass spectra, see the SuSpect [9] and SPheno [10] webpages.
} 
we also include the theoretical uncertainty that we estimate to be $\Delta M_{h} \approx 4 \mathrm{GeV}$ in this case, we obtain the following maximum maximorum bound on the lighter Higgs boson mass in the unconstrained MSSM:

$$
M_{h} \lesssim 152 \mathrm{GeV}
$$

Results for the maximal $M_{h}$ value in the unconstrained pMSSM, based on the two-loop OS calculation implemented in FeynHiggs, have been presented e.g. in Ref. [33], where the upper bound $M_{h} \lesssim 140 \mathrm{GeV}$ can be inferred for $M_{S}=2 \mathrm{TeV}$ and $M_{t}=179.4 \mathrm{GeV}$. A recent update [48], using the latest upper value $M_{t}=182.3 \mathrm{GeV}$, gives $M_{h} \lesssim 143 \mathrm{GeV}$. In both cases, the MSSM parameters are chosen as in the $M_{h}^{\max }$ scenario of Ref. [45], and the theoretical uncertainty is not taken into account. Including the theoretical uncertainty, these results become only a few $\mathrm{GeV}$ lower than the upper bounds discussed above.

\subsubsection{The upper bound on $M_{h}$ in the constrained MSSM}

In the mSUGRA, GMSB and AMSB scenarios, the various parameters which enter the radiative corrections are not all independent, due to the relations between SUSY breaking parameters that are set at the high-energy scale. In addition, the radiative EWSB constraint must be fulfilled for each set of input parameters (in the pMSSM, this is automatic since $M_{A}$ and $\mu$ are used as input parameters). Thus, in contrast to what occurs in the pMSSM, it is not possible to freely tune all relevant weak-scale parameters in order to get a maximal value of $M_{h}$. This should in principle make the analysis more complicated. However, since we have only a small set of input parameters in the constrained case, one can easily perform scans of the parameter space.

For the purpose of illustration, we show in figs. 4 6 how $M_{h}$ depends on some relevant parameters of the mSUGRA, GMSB and AMSB scenarios. The results shown in the figures are obtained with SoftSusy. Fig. 4 shows the value of $M_{h}$ as the background density in the plane $A_{0}-\tan \beta$ of the mSUGRA model. $m_{0}$ and $m_{1 / 2}$ are set to $1 \mathrm{TeV}$ in order to have an average stop mass $M_{S}$ of the order of $2 \mathrm{TeV}$, and $\mu$ is taken positive. In the white region in the upper-left corner of the plot a sparticle squared mass is negative, resulting in an unacceptable scalar minimum. Contours of $M_{S}$ and $M_{H}$ are also shown. Large stop mixing is achieved for large and negative $A_{0}$, around $-4 \mathrm{TeV}$, corresponding to the maximal $M_{h} \approx 128.5 \mathrm{GeV}$ region in the figure.

Fig. 5 shows the value of $M_{h}$ as the background density in the plane $\Lambda-\tan \beta$ of the GMSB model. The messenger mass $M_{\text {mess }}$ is taken equal to $10^{5} \times \Lambda$. Moreover, $N_{\text {mess }}=8$ and $\mu$ is taken positive. It can be seen that the maximal value for $M_{h}$, which is around 123.5 GeV in this example, is obtained for large values of the SUSY-breaking scale $\Lambda$, which correspond to large stop masses.

Finally, in fig. 6] we show $M_{h}$ in the $m_{3 / 2}-\tan \beta$ plane of the AMSB model. The gravitino mass $m_{3 / 2}$ is varied in a range that allows for stop masses of the order of $2 \mathrm{TeV}$, the scalar mass term $m_{0}$ is set to $1 \mathrm{TeV}$, and $\mu$ is taken to be negative. Even in this case, it can be 


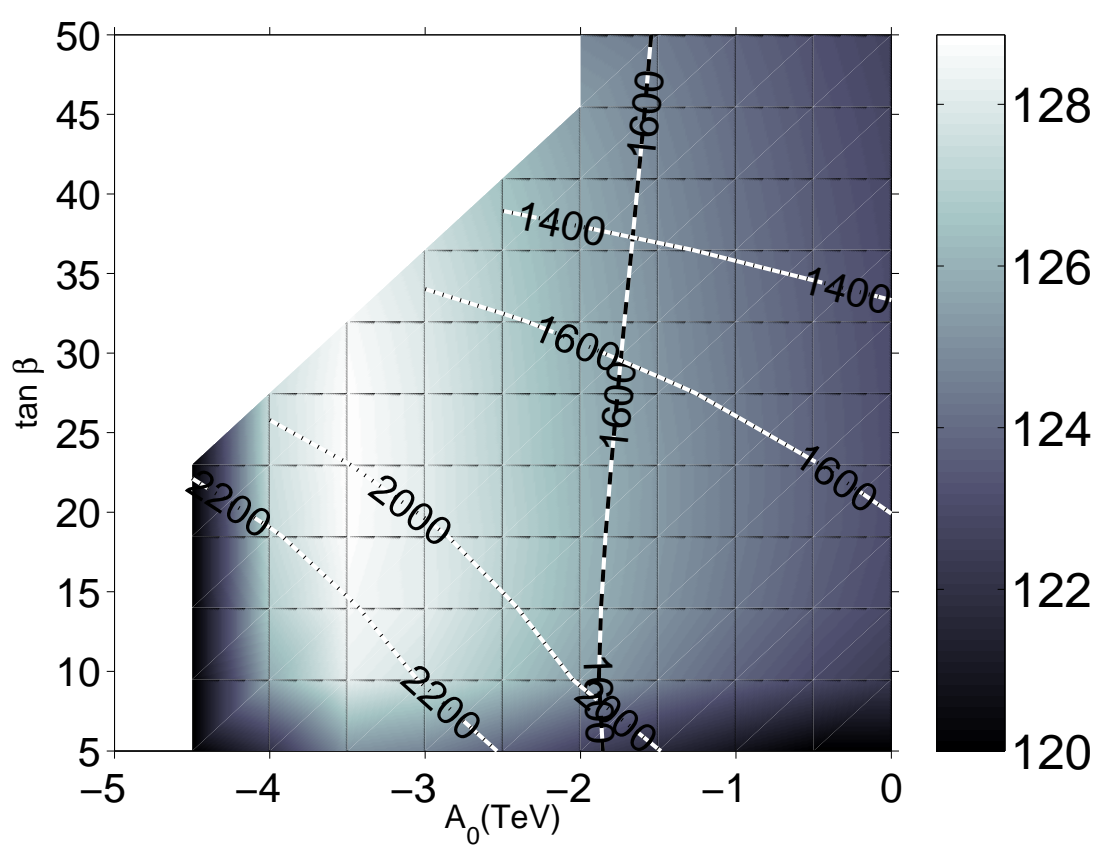

Figure 4: The lighter Higgs boson mass $M_{h}$ in the $\tan \beta-A_{0}$ plane of the mSUGRA scenario. Other input parameters are $m_{0}=m_{1 / 2}=1 \mathrm{TeV}$ and $\mu>0$. The SM input parameters are fixed to their default values. The lighter Higgs boson mass is shown as background density, as measured by the bar on the right. Dashed contours are lines of equal $M_{S}$, dotted contours are lines of equal $M_{H}$. The white region contains scalars with negative squared mass.

seen that $M_{h}$ increases for larger values of $m_{3 / 2}$, which correspond to larger values of the stop masses.

Plots like the ones discussed above can give useful indications about the choice of parameters that leads to the maximal $M_{h}$. However, in order to get a reliable determination of the maximal $M_{h}$ in a given SUSY-breaking scenario it is necessary to scan through the allowed range of values for all of the relevant parameters. Such scans were previously performed, e.g., in Refs. [36, 37, 38]. The most recent among these analyses, Ref. [38, used the combination of FeynHiggs with different private codes [54] for the computation of the MSSM parameters in the various SUSY-breaking scenarios. For convenience, we take the same ranges of soft SUSY-breaking input parameters as in Ref. [38]:

mSUGRA: $\quad 50 \mathrm{GeV} \leq m_{0} \leq 1 \mathrm{TeV}, \quad 50 \mathrm{GeV} \leq m_{1 / 2} \leq 1 \mathrm{TeV}, \quad\left|A_{0}\right| \leq 3 \mathrm{TeV} ;$

GMSB: $\quad 10 \mathrm{TeV} \leq \Lambda \leq 200 \mathrm{TeV}, \quad 1.01 \leq M_{\text {mess }} / \Lambda \leq 10^{5}, \quad 1 \leq N_{\text {mess }} \leq 8 ;$

AMSB: $\quad 20 \mathrm{TeV} \leq m_{3 / 2} \leq 100 \mathrm{TeV}, \quad 50 \mathrm{GeV} \leq m_{0} \leq 2 \mathrm{TeV}$.

Moreover, we require

$$
1 \leq \tan \beta \leq 60
$$

and we allow for both signs of $\mu$. The SM input parameters are fixed to their default values as in eqs. (8) -(9). In particular, we take $M_{t}=178.0 \pm 4.3 \mathrm{GeV}$ [35], whereas Ref. [38] used 


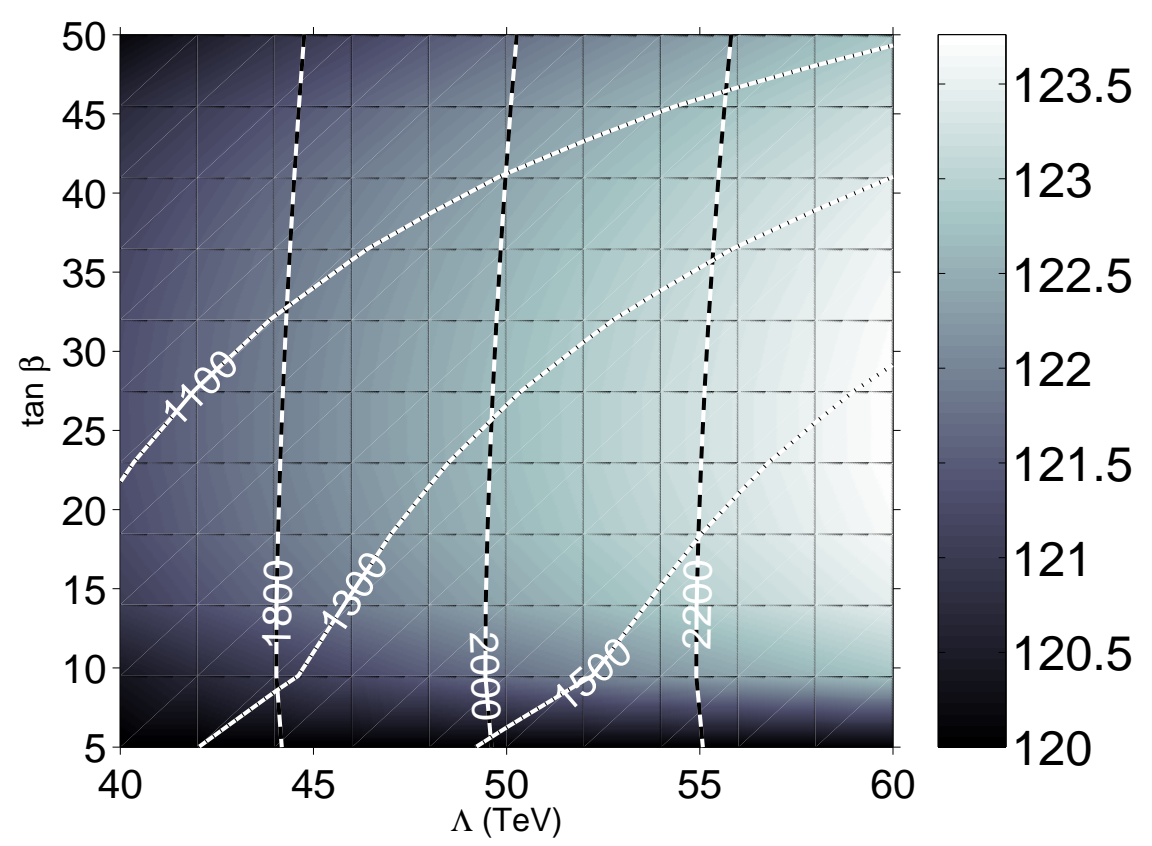

Figure 5: Same as fig. 4 in the $\tan \beta-\Lambda$ plane of the GMSB scenario. Other input parameters are $M_{\text {mess }}=10^{5} \times \Lambda, N_{\text {mess }}=8$ and $\mu>0$.

the old world average $M_{t}=175 \mathrm{GeV}$.

To avoid the need for excessive fine-tuning in the EWSB conditions, we also impose an additional bound on the weak-scale parameters, i.e. that the geometrical average of the stop masses is less than $2 \mathrm{TeV}$,

$$
M_{S}=M_{\mathrm{EWSB}}=\sqrt{m_{\tilde{t}_{1}} m_{\tilde{t}_{2}}}<2 \mathrm{TeV} .
$$

However, it is important to bear in mind that, in the absence of a compelling criterion to define the maximal acceptable amount of fine-tuning, the choice of the upper bound on $M_{S}$ is somehow subjective. This upper bound on $M_{S}$ affects the maximal attainable value of $M_{h}$, as larger values of $M_{S}$ push the value of $M_{h}$ upward.

Taking the range of SUSY-breaking parameters described above and scanning with SoftSusy, SPheno and SuSpect leads to the results for the maximal $M_{h}$ given in table 16, where $M_{t}$ is set to $173.7,178.0$ and $182.3 \mathrm{GeV}$; we also display, for completeness, the upper bounds in the case of the pMSSM.

We display in table 16 only the result of the code that gives larger upper bound on $M_{h}$; the differences between the results of the three codes in the search for the maximal $M_{h}$ can amount to $1 \mathrm{GeV}$, somewhat larger than those found in section 3.2.1 for the SPS scenarios. Apparently, the higher order effects that induce the residual differences among the codes are enhanced for the extreme choices of the SUSY parameters that give rise to the maximal values of $M_{h}$. In addition, in the last column of the table we show our most conservative estimate for the upper bound on the lighter Higgs boson mass in the mSUGRA, GMSB and AMSB scenarios. For this, we take $M_{t}=182.3 \mathrm{GeV}$, the central values for the other 


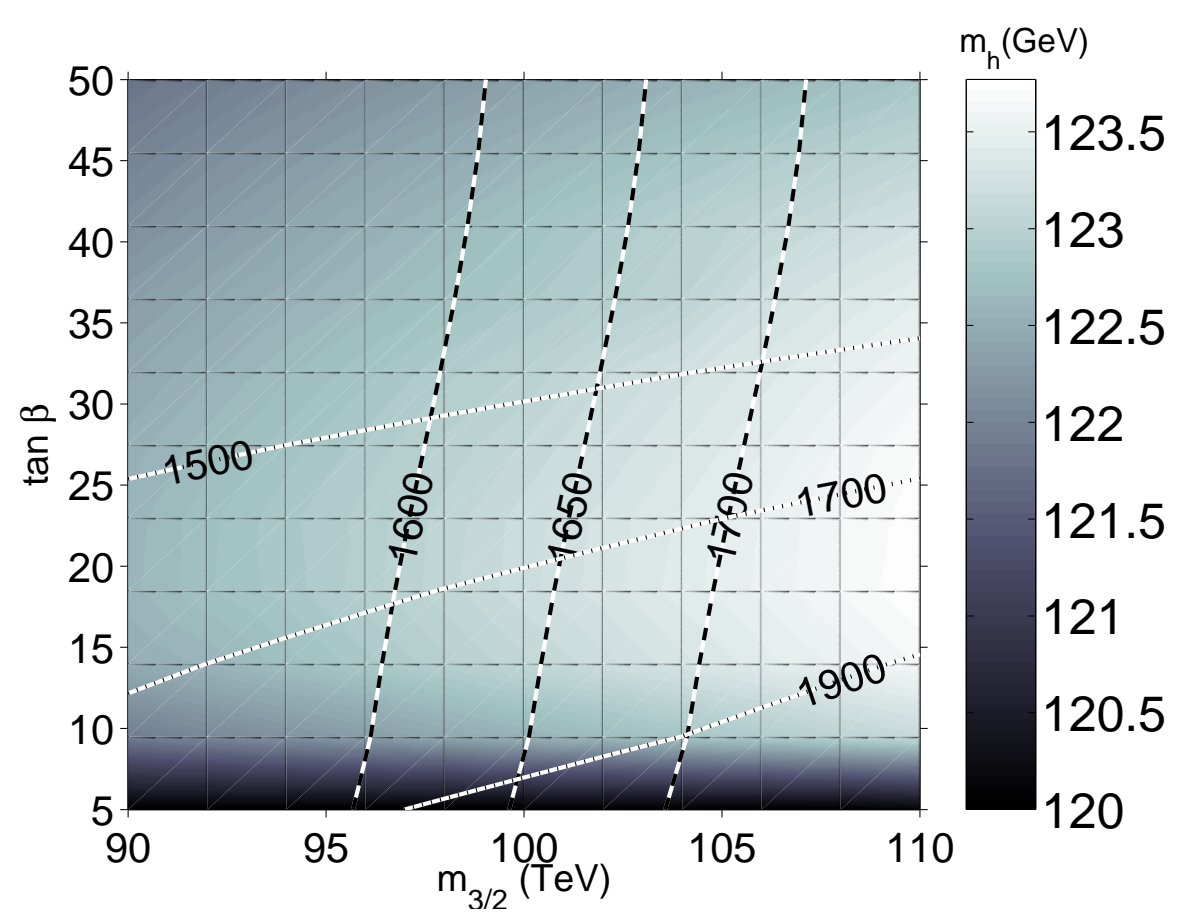

Figure 6: Same as fig. 4 in the $\tan \beta-m_{0}$ plane of the AMSB scenario. Other input parameters are $m_{3 / 2}=100 \mathrm{TeV}$ and $\mu<0$.

SM input parameters and add to the results an uncertainty of approximately $4 \mathrm{GeV}$ (which includes both the theoretical uncertainty as estimated previously, and the much smaller error due to the variation of the remaining SM input parameters).

It can be seen that, for the central value of $M_{t}$, the GMSB model has the tightest bounds upon the maximal lighter Higgs mass, $M_{h} \lesssim 123.7 \mathrm{GeV}$, followed closely by the AMSB model, where the maximal value is obtained for $\mu<0$ (for the central value of the top mass, setting $\mu>0$ would induce a $0.6 \mathrm{GeV}$ decrease in the maximal $M_{h}$ ). The mSUGRA model has a much looser bound, $\sim 5 \mathrm{GeV}$ above the GMSB case, but this bound is more than 10 $\mathrm{GeV}$ smaller than in the case of the pMSSM. This pattern can be qualitatively understood by considering in each model the allowed weak scale values of $A_{t}$, which essentially determines the stop mixing parameter $X_{t}$ and, therefore, the value of $M_{h}$. The GMSB model has $A_{t}=0$ at the relatively low scale $M_{\text {mess }}<2 \times 10^{7} \mathrm{TeV}$, thus its magnitude does not increase greatly in the RG evolution down to $M_{\mathrm{EwSB}}$. A small value of $A_{t}$ implies a smaller value for the stop mixing, therefore producing less high $M_{h}$ (see eq. (11)). The AMSB model has a non-zero $A_{t}$ that is fully predicted at any renormalisation scale in terms of the Yukawa and gauge couplings, and of the overall SUSY breaking scale $m_{3 / 2}$ which determines also the common squark mass $M_{S}$. Thus, the ratio between $X_{t}$ and $M_{S}$ cannot be tuned at will in order to get the maximal value of $M_{h}$. Nevertheless, $X_{t} / M_{S}$ turns out to be sizeable at the weak scale, resulting in a larger value of $M_{h}$ than in the GMSB model. Finally, in the mSUGRA model the only restriction on $A_{t}\left(M_{\mathrm{EWSB}}\right)$ comes from $\left|A_{0}\right|<3 \mathrm{TeV}$, allowing for close to maximal mixing, but only for values of the SUSY scale lower than the value $M_{S}=2 \mathrm{TeV}$ which is used as input in the pMSSM case; if we allow for larger and negative values of $A_{0}$ we can get closer to, but not saturate, the pMSSM bound. In fact, it can be seen from fig. 3 that, 


\begin{tabular}{|l|c|c|c||c|}
\hline$M_{h}^{\max }$ & $M_{t}=173.7 \mathrm{GeV}$ & $M_{t}=178.0 \mathrm{GeV}$ & $M_{t}=182.3 \mathrm{GeV}$ & conservative \\
\hline pMSSM & 138 & 143 & 148 & 152 \\
\hline mSUGRA & 126.2 & 129.0 & 131.7 & 136 \\
GMSB & 120.8 & 123.7 & 126.7 & 131 \\
AMSB & 122.0 & 124.6 & 127.1 & 131 \\
\hline
\end{tabular}

Table 16: Maximal lighter Higgs boson mass (in GeV) in the pMSSM, mSUGRA, GMSB and AMSB scenarios as obtained by the three spectrum generators for three values of the top quark mass. The last column gives the most conservative estimate, where $M_{t}=182.3$ $\mathrm{GeV}$ and a theoretical uncertainty around $4 \mathrm{GeV}$ is added.

in the unconstrained MSSM, the maximal $M_{h}$ is obtained for positive values of $X_{t}$. On the other hand, in the constrained models the RG evolution tends to drive $A_{t}$ (thus, $X_{t}$ ) towards negative values. In order to get at the weak scale a positive $X_{t}$ large enough to obtain the maximal $M_{h}$, we should start from an unreasonably large positive value of $A_{0}$.

Note that table 16 also shows that changing $M_{t}$ by one standard deviation adds or subtracts about $3 \mathrm{GeV}$ to the maximal $M_{h}$ value in each of the cMSSM scenarios, to be compared with the $5 \mathrm{GeV}$ variation discussed previously for the pMSSM.

In Ref [38], the upper bounds $M_{h}<126.6,123.2$ and $124.5 \mathrm{GeV}$ are quoted for the mSUGRA, GMSB and AMSB models, respectively. These results follow the same qualitative pattern as those given in table 16. However, the good agreement found in the GMSB and AMSB scenarios using the central value of $M_{t}$ is to some extent accidental: as discussed above, our $\overline{\mathrm{DR}}$ calculation gives results for $M_{h}$ that are a few $\mathrm{GeV}$ smaller than the ones of the OS calculation in FeynHiggs, but this effect is compensated by the higher central value of $M_{t}$ taken here $\left(M_{t}=178 \mathrm{GeV}\right.$ instead of $\left.M_{t}=175 \mathrm{GeV}\right)$. Also, the authors of Ref. [38] did not impose an upper bound on $M_{S}$, resulting for example in stop masses up to $10 \mathrm{TeV}$ in GMSB (and therefore an enhancement of $M_{h}$ ). Finally, the case $\mu<0$ was not considered in their analysis, which resulted in a reduced upper bound on $M_{h}$ in the AMSB case.

Note that the bounds on $M_{h}$ discussed above will not be in conflict with any phenomenological constraint. Since the obtained SUSY spectra in the cMSSMs is rather heavy, one easily evades the collider experiment bounds on the sparticle and Higgs boson masses and satisfies the indirect constraints from from high-precision measurements such as $(g-2)_{\mu}$, $\mathrm{BR}(b \rightarrow s \gamma)$ and $\Delta \rho$ (the dark matter constraint is not included).

\subsection{The lower bound on $\tan \beta$}

The present LEP2 limit on the mass of the SM Higgs boson, $M_{H^{0}}>114.4 \mathrm{GeV}$ [55], sets strong constraints on the parameter space of the MSSM Higgs sector. In general, a detailed investigation of the Higgs boson production and decay modes would be required to establish 
whether a point in the MSSM parameter space is excluded by the experimental searches. However, in the decoupling regime where $M_{A} \gg M_{Z}$, the lighter $\mathrm{CP}$-even Higgs boson of the MSSM has the same couplings to fermions and gauge bosons as the SM Higgs boson (and hence the same production and decay properties) thus the LEP2 bound can be extrapolated to $M_{h}>114.4 \mathrm{GeV}$. The tree-level value of $M_{h}$ decreases for small values 7 of $\tan \beta$, as can be seen from eq. (10), and the lower experimental bound on $M_{h}$ translates into a lower bound for $\tan \beta$.

In this section, we provide such a bound in the different SUSY breaking scenarios discussed previously. Such investigation is interesting for several reasons:

- An appealing possibility is that the Yukawa couplings of the third generation fermions are unified [e.g., $h_{b}\left(M_{G U T}\right)=h_{\tau}\left(M_{G U T}\right)$ in minimal SU(5)]. In such models, the value of $\tan \beta$ is of critical importance and determines the viability of bottom-tau Yukawa coupling unification [58].

- The experimental determination of the value of $\tan \beta$ is an important measurement to be performed once the supersymmetric and Higgs particles are detected. Unfortunately, such a measurement can be difficult (see Ref. [59] for instance): in most cases the relations between the SUSY or Higgs parameters or observables which can be used for this determination depend on $\cos (2 \beta)$ rather than on $\tan \beta$ itself. This quantity becomes approximately equal to unity as soon as $\tan \beta \geq 3$, leaving little room for an accurate measurement of $\tan \beta$, if low values of $\tan \beta$ are excluded by the LEP2 constraint8. It is thus interesting to see whether there are still viably low values of $\tan \beta$ that allow for a measurement.

- A related reason is that, in the MSSM Higgs sector, the parameter space of the decoupling regime expands for higher values of $\tan \beta$. For $\tan \beta \gtrsim 5$ for instance, the lighter Higgs boson becomes SM-like as soon as $M_{A}$ exceeds the maximal value of $M_{h}$. This is not the case for low values of $\tan \beta$ : even for $A$ boson masses of several hundred $\mathrm{GeV}$, the lighter MSSM Higgs boson has different couplings to fermions and gauge bosons (and thus different decay and production properties) than the SM Higgs boson.

\subsubsection{The lower bound on $\tan \beta$ in the pMSSM}

In the pMSSM, the absolute lower limit on $\tan \beta$ can be obtained by maximising the lighter Higgs mass in such a way that it exceeds the value $M_{h}>114.4 \mathrm{GeV}$ [55] from direct searches at LEP2 (for earlier studies, see e.g. Refs. [63, 33]). Therefore, one can perform the same analysis as for the determination of the maximal $M_{h}$ value discussed in the previous

\footnotetext{
${ }^{7}$ Also, for $\tan \beta<4$, one is close to the infrared quasi fixed point regime of the MSSM [56, where the lighter Higgs boson mass $M_{h}$ becomes smaller [57.

${ }^{8}$ Note that, for high values, $\tan \beta$ could be possibly measured by considering associated production of the heavier MSSM Higgs bosons with bottom quarks [60, tau fusion into Higgs bosons [61] or stau decays into Higgs bosons [62].
} 
subsection, except that here, one looks for the minimal value of $\tan \beta$ which leads to $M_{h}>$ 114.4 GeV. Thus, one has to be in the regime defined by the items (ii)-(iv) of section 4.1, i.e. being in the decoupling regime with a large mixing scenario and using large stop masses. In addition, as in the case of the maximal $M_{h}$, one still has to tune the values of $\mu$ and of the soft SUSY-breaking parameters in order to maximise the radiative corrections.

To obtain the lower bound on the parameter $\tan \beta$, we have performed a scan similar to the one discussed in section 4.1.1, looking for the maximal value of $M_{h}$ corresponding to a given value of $\tan \beta$. In fig. 7, we display the variation of the maximal $M_{h}$ in the pMSSM as a function of $\tan \beta$. The dotted, full and dashed lines show the values of $M_{h}$ for the top mass values $M_{t}=173.7,178.0$ and $182.3 \mathrm{GeV}$, respectively, while the dash-dotted line on the top is for the conservative case where $M_{t}=182.3 \mathrm{GeV}$ is used and a $4 \mathrm{GeV}$ theoretical uncertainty is added to $M_{h}$.

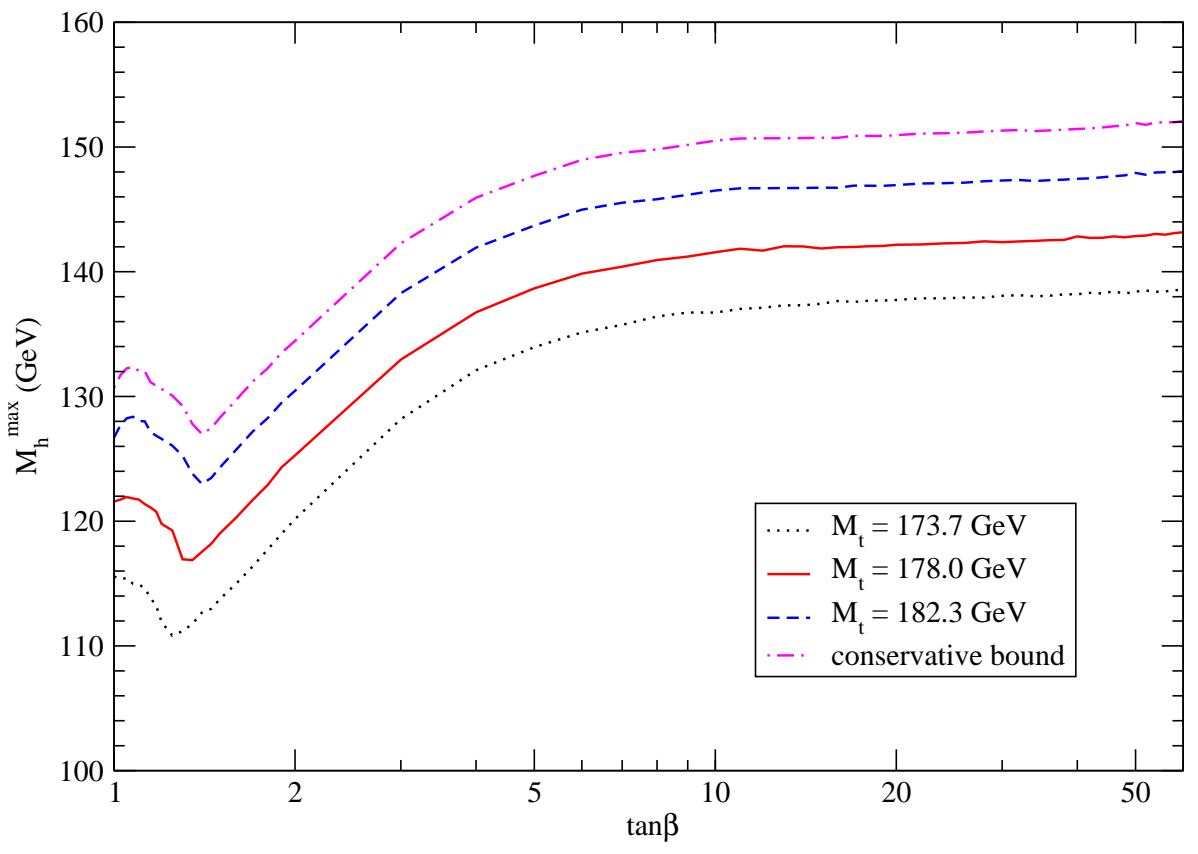

Figure 7: Upper bound on the lighter Higgs boson mass $M_{h}$ in the pMSSM as a function of $\tan \beta$, as obtained from a full scan of the parameter space. The dotted, full and dashed lines correspond to the top mass values $M_{t}=173.7,178.0$ and $182.3 \mathrm{GeV}$, respectively, while the dash-dotted line on the top is for the conservative case where $M_{t}=182.3 \mathrm{GeV}$ is used and a $4 \mathrm{GeV}$ theoretical uncertainty is added to $M_{h}$.

As can be seen, for the default value $M_{t}=178 \mathrm{GeV}$ the LEP2 bound of $114.4 \mathrm{GeV}$ on $M_{h}$ is always satisfied and therefore, for this top quark mass value, no empirical bound on $\tan \beta$ (provided that it is larger than unity) can be derived in the pMSSM. This is of course 
also the case for the larger value of $M_{t}=182.3 \mathrm{GeV}$, and a fortiori for the conservative case where a theoretical uncertainty is taken into account, where all values $1 \leq \tan \beta \leq 60$ are allowed by the LEP2 constraint. Only in the case of a lighter top quark, $M_{t}=173.7$ $\mathrm{GeV}$, the range $1.1 \lesssim \tan \beta \lesssim 1.6$ is excluded if the LEP2 bound $M_{h} \geq 114.4 \mathrm{GeV}$ is to be satisfied. However, if a theoretical uncertainty of $4 \mathrm{GeV}$ on $M_{h}$ is included (meaning, in practice, that the LEP2 Higgs mass bound translates to the bound $M_{h} \geq 110.4 \mathrm{GeV}$ on the prediction obtained without including the theoretical uncertainty) no bound on the parameter $\tan \beta$ can be obtained from the LEP2 constraint.

The local maximum around $\tan \beta=1.2$ for the maximal $M_{h}$ in fig. 7 is due to the fact that the top Yukawa coupling is maximised by the threshold corrections in that point. On the other hand, in the large $\tan \beta$ regime one recovers the maximal $M_{h}$ values discussed in the previous section, as shown in the right-hand side of the figure. In particular, one sees that the upper bound on the lighter Higgs boson, in the conservative case where $M_{t}=182.3$ $\mathrm{GeV}$ and a $4 \mathrm{GeV}$ theoretical uncertainty on $M_{h}$ is added, is slightly above $150 \mathrm{GeV}$.

\subsubsection{The lower bound on $\tan \beta$ in the constrained MSSM}

In the constrained MSSM scenarios, we parameterise the 95\% C.L. bounds from LEP2 in the $\sin ^{2}(\beta-\alpha)-M_{h}$ parameter space [55], rather than just using the lower bound on $M_{h}$, in case the decoupling regime is not obtained. In practice, the lowest values of $\tan \beta$ that saturate the LEP2 limit are indeed obtained in the decoupling regime. To reduce the volume of the space that is scanned over, we use the parameter ranges as described in section 4.1 for the mSUGRA, GMSB and AMSB cases. The results are summarised for these three cases in table 17 where the values $M_{t}=173.7,178.0 \mathrm{GeV}$ and $182.3 \mathrm{GeV}$ are used, while for the other SM inputs we used the default values given in eqs. (8)-(9). The last column shows the most conservative lower bounds, obtained by adding a theoretical uncertainty of $4 \mathrm{GeV}$ to the prediction of the lighter Higgs boson mass. For completeness, we also include in the first row the results in the pMSSM case discussed previously. In each entry of the table, we quote the result obtained with the code that gives the weakest lower bound on $\tan \beta$.

\begin{tabular}{|l|c|c|c||c|}
\hline $\tan \beta^{\min }$ & $M_{t}=173.7 \mathrm{GeV}$ & $M_{t}=178.0 \mathrm{GeV}$ & $M_{t}=182.3 \mathrm{GeV}$ & conservative \\
\hline pMSSM & 1.6 & - & - & - \\
\hline mSUGRA & 2.8 & 2.4 & 2.1 & 1.9 \\
GMSB & 4.2 & 3.3 & 2.7 & 2.2 \\
AMSB & 3.7 & 3.1 & 2.7 & 2.3 \\
\hline
\end{tabular}

Table 17: Lower bounds on the parameter $\tan \beta$ in the pMSSM, mSUGRA, GMSB and AMSB scenarios for three values of the pole top mass. The last column gives the most conservative estimate, where we use $M_{t}=182.3 \mathrm{GeV}$ and add a $4 \mathrm{GeV}$ theoretical uncertainty on $M_{h}$. 
One can see from the table that, as expected, the minimal values of $\tan \beta$ follow the same pattern as in the evaluation of the maximal $M_{h}$ value. The bounds are most stringent in the GMSB scenario, closely followed by the AMSB scenario, and finally by the mSUGRA scenario. In all cases the bounds are much stronger than in the pMSSM. In fact, contrary to the pMSSM case, in the constrained scenarios there is always a significant bound on the parameter $\tan \beta$ even for the largest value of $M_{t}$, $\tan \beta \gtrsim 2.1-2.7$, and when a $4 \mathrm{GeV}$ theoretical uncertainty on $M_{h}$ is included, $\tan \beta \gtrsim 1.9-2.3$. This is merely a consequence of the $\sim 14 \mathrm{GeV}$ difference between the maximal values of $M_{h}$ in the constrained and unconstrained cases.

In Ref. [38] the lower bounds $\tan \beta>2.9,3.1$ and 3.8 for the mSUGRA, GMSB and AMSB scenarios, respectively, are quoted. In contrast to our findings, summarised in table 17. the bound quoted in Ref. [38] for the AMSB scenario is stronger than the one quoted for the GMSB scenario, presumably due to the fact that the authors did not consider the case $\mu<0$ in their analysis 9. On the other hand, our results for the mSUGRA and GMSB scenarios, using the central value of $M_{t}$, are relatively close to the results of Ref. [38]. This is again due to a compensation between the tendency of the $\overline{\mathrm{DR}}$ calculation to give lower values of $M_{h}$ than the OS calculation (thus, stronger bounds on $\tan \beta$ ), and the increased value of $M_{t}$, resulting into higher values of $M_{h}$ (thus, weaker bounds on $\tan \beta$ ).

\subsection{Implications for the decays of the Higgs bosons}

As discussed in the previous subsections, in the most conservative case where the top quark mass is set to $M_{t}=182.3 \mathrm{GeV}$ and a theoretical uncertainty of $4 \mathrm{GeV}$ is added to $M_{h}$, the upper bound on the lighter Higgs boson mass in the unconstrained MSSM can reach the level of $150 \mathrm{GeV}$, and there is no lower bound on the parameter $\tan \beta$ from the negative Higgs boson searches at LEP2. Such large values of $M_{h}$ and small values of $\tan \beta$ would have a decisive impact on the decays of the MSSM Higgs bosons (and also on their production rates) and thus on their experimental detection at future high-energy colliders, in particular at the LHC, as will be briefly discussed below.

In the decoupling regime, the lighter Higgs boson $h$ behaves approximately like the SM Higgs particle. In particular, it has the same couplings to fermions and gauge bosons, thus the same branching fractions and production cross sections, provided that the SUSY particles are heavy enough to make their contribution to the loop induced decays safely negligible [65]. In fig. 8, obtained with the code HDECAY, we display the branching ratios of the SM Higgs boson (equivalent to those of $h$ in the decoupling regime) as a function of its mass.

As can be seen, while the $h \rightarrow b \bar{b}$ decay mode is dominant for masses below $M_{h} \sim 135$ $\mathrm{GeV}$, it is the decay $h \rightarrow W W$ (with one of the gauge boson being off-shell) which becomes the most important one above this Higgs mass value. In particular, for Higgs boson masses

\footnotetext{
${ }^{9}$ We have checked that in the considered scenarios the SUSY contribution is within the $2 \sigma$ range for $(g-2)_{\mu}$ (see Ref. 64 for a recent analysis).
} 
close to the upper bound, $M_{h} \sim 150 \mathrm{GeV}$, the decay into $W$ boson pairs reaches a branching ratio of the order of $70 \%$. Contrary to the $b \bar{b}$ decay mode, which suffers from a huge hadronic background at the LHC, the $W W$ decay mode is much cleaner if one considers the leptonic decays of one or two $W$ bosons, and therefore becomes the most promising detection channel at the LHC [66].

Because the partial decay width of the lighter Higgs boson into $W W$ final states is enhanced by the gain in phase space, almost all of the other decay modes will be suppressed. The relatively clean decays into $\tau$-lepton pairs, which are a useful signal when the lighter Higgs boson is produced in the fusion of two gauge bosons at the LHC $p p \rightarrow q q h$ with $h \rightarrow \tau^{+} \tau^{-}$[66], can be suppressed to the level of $2 \%$ and this detection channel might therefore be disfavoured. The rare but very clean decay of the lighter Higgs boson into two photons is not strongly affected, being at the level of a few per mille, and still constitutes a viable detection channe 10 .

Another consequence of a large $M_{h}$ would be that, because of the increase of the phase space, the branching ratio for the decay of the lighter Higgs boson into $Z$ boson pairs can reach the level of $10 \%$. This would make the detection channel $h \rightarrow Z Z^{*} \rightarrow 4 \ell^{ \pm}$more important at the LHC, and the larger number of events could eventually allow for the determination of the spin-parity quantum numbers of the Higgs particle from the study of the angular correlations between the final state leptons [67].

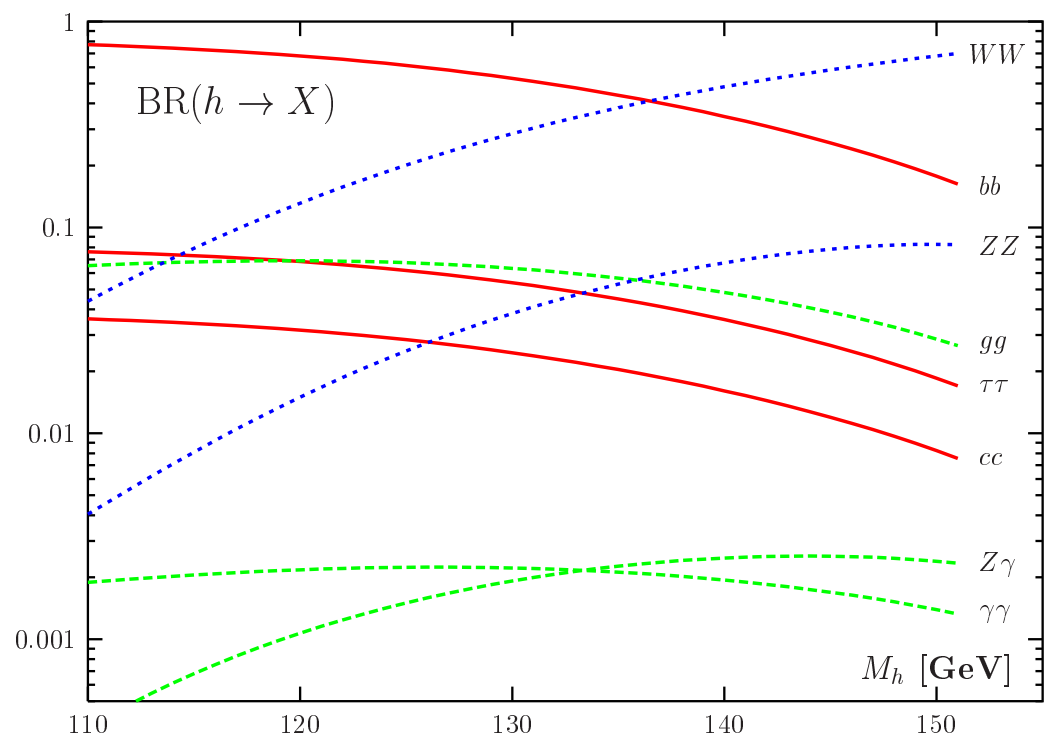

Figure 8: The branching fractions of the SM Higgs boson (equivalent to those of the lighter MSSM Higgs boson $h$ in the decoupling regime) as a function of its mass.

Let us now turn to the consequences of the constraints on the parameter $\tan \beta$ obtained from the negative searches of the MSSM Higgs bosons at LEP2. First of all, we recall that the

\footnotetext{
10 The branching ratio for this decay is not suppressed because it is dominantly mediated by $W$ boson loops, whose contribution becomes maximal near the $W W$ threshold.
} 
lower $\tan \beta$ bounds discussed in the previous subsection, have been derived in the decoupling regime where the pseudoscalar Higgs boson is rather heavy, $M_{A}=2 \mathrm{TeV}$. To derive this constraint outside the decoupling regime, one can no longer use the LEP2 bound on the SM Higgs boson mass, but rather, the $\sin ^{2}(\beta-\alpha)-M_{h}$ parameter space excluded by the LEP collaborations [55] in the search of the MSSM Higgs bosons in all production channels. A detailed analysis of the lower bound on $\tan \beta$ for any value of $M_{A}$ and its impact on the decays of the MSSM Higgs bosons and on their production rates at the various high-energy colliders is beyond the scope of this paper. Nevertheless, a rough analysis shows that, in the conservative case discussed above, values of $\tan \beta \sim 1.8$ are still allowed for $M_{A}$ values as low as $200 \mathrm{GeV}$, which leads to the following qualitative conclusions:

- The decays of the three neutral MSSM Higgs bosons into $b \bar{b}$ and $\tau^{+} \tau^{-}$final states may not be too strongly enhanced. The lighter MSSM Higgs boson will then behave more like the SM Higgs particle even outside the decoupling regime.

- The decays of the heavier CP-even Higgs bosons into two gauge bosons and into two lighter Higgs bosons would be less suppressed. In particular, if the decay $H \rightarrow h h$ is allowed it could lead to the possibility of measuring the trilinear Hhh coupling.

- The decays of the pseudoscalar and charged Higgs bosons into a gauge boson and the lighter Higgs boson, $A \rightarrow h Z$ and $H^{ \pm} \rightarrow h W^{ \pm}$, could provide interesting detection signals.

A detailed analysis of these features will be presented in a forthcoming publication [68].

\section{Conclusions}

In this paper, we provided a detailed analysis of the Higgs sector of the Minimal Supersymmetric extension of the Standard Model, both in the constrained cases such as mSUGRA, GMSB and AMSB and in the unconstrained case, where 22 parameters are allowed to be free at the EWSB scale. We have provided a very precise determination of the masses of the neutral Higgs bosons including all the presently available radiative corrections and using the latest value of the top quark mass, as recently measured at the Tevatron.

In three public RGE codes which evaluate the supersymmetric particle spectrum, SoftSusy, SPheno and SuSpect, we have implemented all of the available radiative corrections for the Higgs boson masses and for the effective potential, in the $\overline{\mathrm{DR}}$ renormalisation scheme which is more natural for these spectrum generators. In particular, we have incorporated the full set of one-loop radiative corrections to the Higgs self-energies and to the tadpoles, including the contributions of all SUSY particles and taking into account the external momentum dependence. We have also included the dominant two-loop radiative corrections at vanishing external momentum, i.e. the corrections which involve the strong gauge coupling and the Yukawa couplings of the third generation fermions. To complete this picture, we have 
derived the missing radiative corrections controlled by the $\tau$ lepton Yukawa coupling, which however turned out to be numerically small.

We have performed a detailed comparison of the three programs, in six SPS scenarios for the mSUGRA, GMSB and AMSB cases as well as in three scenarios for the unconstrained MSSM. We have found a very good agreement among the results of the three codes: to within half $\mathrm{GeV}$ for the mass of the lighter $\mathrm{CP}$-even Higgs boson and within 1\% for the heavier $\mathrm{CP}$-even and $\mathrm{CP}$-odd Higgs bosons. We therefore conclude that the three codes provide now a reliable and very precise determination of the neutral Higgs boson masses, both in constrained and unconstrained MSSM scenarios.

We have then studied the effects of the various radiative corrections on the Higgs boson masses in constrained scenarios and in the $\overline{\mathrm{DR}}$ scheme. The corrections due to the strong interactions, $\mathcal{O}\left(\alpha_{t} \alpha_{s}+\alpha_{b} \alpha_{s}\right)$, increase the lighter Higgs boson mass by up to $6 \mathrm{GeV}$, while the pure Yukawa corrections due to the top and bottom quarks, $\mathcal{O}\left(\alpha_{t}^{2}+\alpha_{t} \alpha_{b}+\alpha_{b}^{2}\right)$, can decrease it by $1 \mathrm{GeV}$; the corrections due to the $\tau$-Yukawa couplings, $\mathcal{O}\left(\alpha_{\tau}^{2}+\alpha_{b} \alpha_{\tau}\right)$, can be safely neglected. The impact of some higher order effects, such as those originating from the inclusion of the two-loop standard QCD and one-loop electroweak corrections to the top quark mass, the resummation of the SUSY-QCD corrections in the bottom quark mass, the use of the two-loop RGEs for the sfermion masses and the use of the pole Higgs boson masses in the one-loop corrections, has also been analysed. These higher order effects can alter the results by up to half a GeV for $M_{h}$ and up to a few percent in $M_{H}$.

A detailed study of the theoretical and experimental uncertainties in the determination of the CP-even Higgs boson masses has been performed in both the constrained and unconstrained MSSM scenarios. The comparison between the three RGE codes and the program FeynHiggs, where the MSSM Higgs boson masses are evaluated in the On-Shell renormalisation scheme, gives an estimate of the scheme dependence of the predictions. This dependence is in general of the order of $2 \mathrm{GeV}$, but can reach the level of $4-5 \mathrm{GeV}$ in some cases, such as in the pMSSM scenario with large mixing in the stop sector. The dependence on the scale at which the effective scalar potential is minimised and the radiative corrections are computed is significantly improved by the inclusion of the two-loop radiative corrections, leaving a variation of less than $3 \mathrm{GeV}$ on $M_{h}$ (apart from the case of the pMSSM scenario with large stop mixing, where the variation is larger). The effect of approximating the external momentum in the two-loop Higgs boson self-energies to zero rather than to the mass of the Higgs boson has been estimated to lead to a shift of less than half a $\mathrm{GeV}$ on $M_{h}$ and to an even smaller shift on $M_{H}$.

All of these shifts indicate the size of the higher order corrections that are left uncomputed, thus they are a measure of the theoretical uncertainty which affects the calculation. As a summary, we conclude that these effects induce a theoretical uncertainty on the mass of the lighter Higgs boson of $3-4 \mathrm{GeV}$ in the constrained MSSM and up to $5 \mathrm{GeV}$ in the unconstrained MSSM, in particular when the mixing in the stop sector is such that $M_{h}$ is maximised. The uncertainties on $M_{h}$ due to the experimental errors in the measurement of the SM input parameters are approximately of the same size. Indeed, a variation of the 
top quark mass within its $1 \sigma$ range, $M_{t}=178.0 \pm 4.3 \mathrm{GeV}$, leads to a shift of about \pm 2 to $\pm 4 \mathrm{GeV}$ on $M_{h}$, depending on the scenario. The shifts on $M_{h}$ from the errors in the measurement of $m_{b}$ and $\alpha_{s}$ are less than half a GeV, while the error on $\alpha$ has a negligible impact. Thus, both a theoretical and an experimental effort are needed to attain the level where the lighter Higgs boson mass can be predicted with a precision of less than $100 \mathrm{MeV}$, i.e. the level of precision at which this mass can be measured at the future colliders.

Finally, using the RGE codes, we have performed a phenomenological study of the impact of the radiative corrections, as well as the theoretical and experimental uncertainties, on the Higgs sector of the constrained and unconstrained MSSM. In particular, using the latest Tevatron value of the top quark pole mass, $M_{t}=178.0 \pm 4.3 \mathrm{GeV}$, we have derived the maximal value of $M_{h}$ in the MSSM, as well as the minimal value of the parameter $\tan \beta$ that is allowed by the negative search of the MSSM Higgs bosons at LEP2. In the unconstrained MSSM, we get an upper bound $M_{h} \lesssim 143 \mathrm{GeV}$ and we show that all values of $\tan \beta$ are allowed by LEP2 constraints. In the constrained MSSM scenarios, as a result of the chosen ranges for the soft SUSY-breaking parameters and of the relations among them, the bounds on $M_{h}$ are stricter than in the pMSSM: 129.0, 123.7 and $124.6 \mathrm{GeV}$ in the mSUGRA, GMSB and AMSB cases respectively. Similarly, in the constrained scenarios it is possible to derive lower bounds on $\tan \beta: 2.4,3.3$ and 3.1 in the mSUGRA, GMSB and AMSB cases respectively. If the upper value of the top quark mass, $M_{t}=182.3 \mathrm{GeV}$, is used, and an estimated $4 \mathrm{GeV}$ theoretical uncertainty on $M_{h}$ is included, one arrives at the conservative upper bound of $M_{h} \lesssim 152 \mathrm{GeV}$ for the value of the lighter Higgs boson in the unconstrained MSSM. Such a bound has important implications for searches and phenomenology of the MSSM Higgs bosons.

\section{Note added}

During the final stages of the preparation of the present work, a paper appeared [69], where a two-loop computation of the leading contributions to the MSSM Higgs boson self-energies for arbitrary external momentum is presented. In the numerical examples presented in Ref. [69], the two-loop momentum-dependent corrections to the lighter Higgs boson mass $M_{h}$ are of the order of a few hundred $\mathrm{MeV}$, while the corresponding corrections to the heavier Higgs boson mass $M_{H}$ are considerably smaller. These results are in good agreement with our empirical estimates given in section 3.3.3. We plan to include the results of Ref. [69] in our spectrum calculators as soon as they become available in a suitable form.

\section{Acknowledgements}

We would like to thank M. Drees, S. Heinemeyer, W. Hollik and G. Weiglein for useful discussions, and K. Desch for providing us with LEP2 Higgs data. This work was partially supported by the European Community's Human Potential Programme HPRN-CT-2000- 
00149 (Collider Physics). W. P. is supported by a Spanish MCyT Ramon y Cajal contract and partly by the Swiss "Nationalfonds". P. S. thanks LAPTH and LPMT for hospitality offered while part of this work was carried out.

\section{Appendix A: EWSB conditions and Higgs boson masses}

In this appendix we provide for completeness the general formulae for the computation of the radiative corrections to the EWSB conditions and to the Higgs boson masses in the MSSM, following the notation of Ref. [16]. We also explain how to adapt the formulae of Refs. [23, 25, 26, 28] to the same notation.

Once the requirement of correct EWSB is imposed, providing input values for the $Z$ boson mass and $\tan \beta=v_{2} / v_{1}$ [where $v_{i}(i=1,2)$ are the vacuum expectation values of the neutral Higgs fields], the minimisation conditions on the MSSM effective potential translate into conditions for the parameters $\mu^{2}$ and $B$ :

$$
\begin{aligned}
\mu^{2} & =-\frac{m_{Z}^{2}}{2}-\frac{1}{2} \tan 2 \beta\left[\left(m_{H_{1}}^{2}-\frac{t_{1}}{v_{1}}\right) \cot \beta-\left(m_{H_{2}}^{2}-\frac{t_{2}}{v_{2}}\right) \tan \beta\right], \\
B & =-\frac{m_{Z}^{2}}{2} \sin 2 \beta-\frac{1}{2} \tan 2 \beta\left(m_{H_{1}}^{2}-\frac{t_{1}}{v_{1}}-m_{H_{2}}^{2}+\frac{t_{2}}{v_{2}}\right),
\end{aligned}
$$

where $m_{Z}$ is the running mass for the $Z$ boson defined in eq. (2),$m_{H_{i}}^{2}(i=1,2)$ are the soft SUSY-breaking mass terms for the two Higgs doublets and $t_{i}$ are the tadpole corrections, corresponding to diagrams with one incoming Higgs field. The squared physical masses for the $\mathrm{CP}-$ odd Higgs boson $A$ and for the charged Higgs boson $H^{ \pm}$can be computed as:

$$
\begin{aligned}
M_{A}^{2} & =2 B / \sin 2 \beta-\operatorname{Re} \Pi_{A A}\left(M_{A}^{2}\right)+\sin ^{2} \beta \frac{t_{1}}{v_{1}}+\cos ^{2} \beta \frac{t_{2}}{v_{2}}, \\
M_{H^{ \pm}}^{2} & =M_{A}^{2}+M_{W}^{2}+\operatorname{Re}\left[\Pi_{A A}\left(M_{A}^{2}\right)-\Pi_{H^{+} H^{-}}\left(M_{H^{ \pm}}^{2}\right)+\Pi_{W W}^{T}\left(M_{W}^{2}\right)\right],
\end{aligned}
$$

where $M_{W}$ is the physical $W$ boson mass, and $\Pi_{A A}\left(M_{A}^{2}\right), \Pi_{H^{+} H^{-}}\left(M_{H^{ \pm}}^{2}\right)$ and $\Pi_{W W}^{T}\left(M_{W}^{2}\right)$ are the self-energies for the $A, H^{ \pm}$and $W$ bosons, respectively, each computed at a squared external momentum equal to the squared physical mass of the corresponding particle.

Finally, the squared physical masses for the CP-even Higgs bosons, $M_{h}^{2}$ and $M_{H}^{2}$, correspond to the real part of the poles of the $2 \times 2$ propagator matrix, i.e. the two solutions of the equation:

$$
\operatorname{Det}\left[p_{i}^{2} \mathrm{I}-\mathcal{M}^{2}\left(p_{i}^{2}\right)\right]=0,
$$

where the radiatively corrected mass matrix for the $\mathrm{CP}$-even Higgs fields is:

$$
\mathcal{M}^{2}\left(p^{2}\right)=\left(\begin{array}{cc}
m_{Z}^{2} \cos ^{2} \beta+m_{A}^{2} \sin ^{2} \beta-\Pi_{11}\left(p^{2}\right)+\frac{t_{1}}{v_{1}} & -\left(m_{Z}^{2}+m_{A}^{2}\right) \sin \beta \cos \beta-\Pi_{12}\left(p^{2}\right) \\
-\left(m_{Z}^{2}+m_{A}^{2}\right) \sin \beta \cos \beta-\Pi_{12}\left(p^{2}\right) & m_{Z}^{2} \sin ^{2} \beta+m_{A}^{2} \cos ^{2} \beta-\Pi_{22}\left(p^{2}\right)+\frac{t_{2}}{v_{2}}
\end{array}\right) .
$$


In the above formula, $m_{A}^{2}=2 B / \sin 2 \beta$ is the squared running mass for the $A$ boson as defined in eq. (7), and $\Pi_{i j}\left(p^{2}\right)(i, j=1,2)$ are the self-energies of the CP-even components of the Higgs fields. In SoftSusy, SPheno and SuSpect an iterative procedure is employed for solving eq. (A5). The Higgs mixing angle $\alpha$, instead, is defined in the codes as the angle that diagonalises $\mathcal{M}^{2}\left(p^{2}\right)$ for $p^{2}=M_{h}^{2}$.

The explicit formulae for the one-loop parts of the self-energies and tadpole diagrams appearing in eqs. (A1)-(A6) can be found in Ref. 16]. In addition, SoftSusy, SPheno and SuSpect employ the formulae given in Refs. [23, 25, 26, 28] for the leading two-loop corrections to the tadpoles and the neutral Higgs boson masses.

The computations in Refs. [23, 25, 26, 28] are performed in the effective potential approach, which implies that the approximation of zero external momentum is used in the two-loop self-energies. Also, the formulae of Refs. [23, 25, 26, 28] assume that the tree-level mass matrix for the $\mathrm{CP}$-even Higgs bosons is expressed in terms of the physical $A$-boson mass, $M_{A}$, whereas in eq. (A6) the mass matrix is expressed in terms of the running mass $m_{A}$. It is thus necessary to rearrange the formulae of Refs. [23, 25, 26, 28] for the corrections to the Higgs tadpoles, $\Sigma_{i}$, to the $\mathrm{CP}$-odd Higgs mass, $\Delta m_{A}^{2}$, and to the CP-even Higgs mass matrix, $\left(\Delta \mathcal{M}_{S}^{2}\right)^{\text {eff }}$, in order to adapt them to the notation of Ref. [16]. In particular, the following equalities hold:

$$
\begin{aligned}
\Sigma_{i} & =-\frac{t_{i}}{v_{i}}(i=1,2), \\
\Delta m_{A}^{2} & =-\operatorname{Re} \Pi_{A A}(0)+\sin ^{2} \beta \frac{t_{1}}{v_{1}}+\cos ^{2} \beta \frac{t_{2}}{v_{2}} \\
\Delta m_{A}^{2} \sin ^{2} \beta+\left(\Delta \mathcal{M}_{S}^{2}\right)_{11}^{\mathrm{eff}} & =-\operatorname{Re} \Pi_{11}(0)+\frac{t_{1}}{v_{1}} \\
-\Delta m_{A}^{2} \sin \beta \cos \beta+\left(\Delta \mathcal{M}_{S}^{2}\right)_{12}^{\mathrm{eff}} & =-\operatorname{Re} \Pi_{12}(0), \\
\Delta m_{A}^{2} \cos ^{2} \beta+\left(\Delta \mathcal{M}_{S}^{2}\right)_{22}^{\mathrm{eff}} & =-\operatorname{Re} \Pi_{22}(0)+\frac{t_{2}}{v_{2}} .
\end{aligned}
$$

\section{Appendix B: Formulae for the $\mathcal{O}\left(\alpha_{b} \alpha_{\tau}\right)$ corrections}

We present in this appendix the explicit analytical formulae for the two-loop $\mathcal{O}\left(\alpha_{b} \alpha_{\tau}\right)$ corrections to the MSSM Higgs masses and tadpoles in the $\overline{\mathrm{DR}}$ renormalisation scheme. We follow very closely the lines of Refs. [23, 25, 24, 26, 28], to which we refer the reader for further details. The following results complete the computation of the two-loop corrections to the Higgs masses and tadpoles controlled by the third-family Yukawa couplings (the inclusion of the $\mathcal{O}\left(\alpha_{\tau}^{2}\right)$ corrections was discussed in Ref. [26]).

If we express the MSSM effective potential $V_{\text {eff }}$ in terms of $\overline{\mathrm{DR}}$-renormalised fields and parameters, the two-loop contribution to $V_{\text {eff }}$ involving both the bottom and tau Yukawa 
couplings $h_{b}$ and $h_{\tau}$ reads, in units of $N_{c} /\left(16 \pi^{2}\right)^{2}$,

$$
\Delta V_{b \tau}=\frac{h_{b} h_{\tau}}{2} s_{2 \bar{\theta}_{b}} s_{2 \bar{\theta}_{\tau}} c_{\tilde{\varphi}_{b}-\tilde{\varphi}_{\tau}}\left[\hat{J}\left(m_{\tilde{b}_{1}}^{2}, m_{\tilde{\tau}_{1}}^{2}\right)-\hat{J}\left(m_{\tilde{b}_{2}}^{2}, m_{\tilde{\tau}_{1}}^{2}\right)-\hat{J}\left(m_{\tilde{b}_{1}}^{2}, m_{\tilde{\tau}_{2}}^{2}\right)+\hat{J}\left(m_{\tilde{b}_{2}}^{2}, m_{\tilde{\tau}_{2}}^{2}\right)\right]
$$

where: $c_{\phi} \equiv \cos \phi$ and $s_{\phi} \equiv \sin \phi ; m_{\tilde{q}_{i}}^{2}(q=\tau, b)$ are the field-dependent squark masses; $\bar{\theta}_{\tilde{q}}$ is the field-dependent squark mixing angle, defined in such a way that $0 \leq \bar{\theta}_{\tilde{q}}<\pi / 2$ (to be contrasted with the usual field-independent mixing angle $\theta_{\tilde{q}}$, such that $-\pi / 2 \leq \theta_{\tilde{q}}<\pi / 2$ ); $\widetilde{\varphi}_{q}$ is the phase in the off-diagonal element of the squark mass matrix. For the explicit Higgs field dependence of these parameters, see Refs. [23, 25]. Finally, the $\overline{\mathrm{DR}}$-subtracted two-loop integral $\hat{J}$, with $Q^{2}$ being the renormalisation scale, is defined as

$$
\hat{J}\left(m_{\tilde{q}_{1}}^{2}, m_{\tilde{q}_{2}}^{2}\right)=m_{\tilde{q}_{1}}^{2} m_{\tilde{q}_{2}}^{2}\left(1-\ln \frac{m_{\tilde{q}_{1}}^{2}}{Q^{2}}\right)\left(1-\ln \frac{m_{\tilde{q}_{2}}^{2}}{Q^{2}}\right),
$$

Exploiting the field-dependence of the squark masses and mixing angles, we can express the tau/bottom corrections to the $\mathrm{CP}$-even Higgs mass matrix, $\left(\Delta \mathcal{M}_{S}^{2}\right)^{\text {eff }}$, to the Higgs tadpoles, $\Sigma_{i}$, and to the $\mathrm{CP}$-odd Higgs mass, $\Delta m_{A}^{2}$, (see appendix A and Ref. [26] for the various definitions) as

$$
\begin{aligned}
& \left(\Delta \mathcal{M}_{S}^{2}\right)_{11}^{\text {eff }}=2 h_{\tau}^{2} m_{\tau}^{2} F_{1}^{\tau}+2 h_{\tau}^{2} A_{\tau} m_{\tau} s_{2 \theta_{\tau}} F_{2}^{\tau}+\frac{1}{2} h_{\tau}^{2} A_{\tau}^{2} s_{2 \theta_{\tau}}^{2} F_{3}^{\tau}+2 h_{\tau} h_{b} m_{b} A_{\tau} s_{2 \theta_{\tau}} F_{4}^{\tau} \\
& +2 h_{b}^{2} m_{b}^{2} F_{1}^{b}+2 h_{b}^{2} A_{b} m_{b} s_{2 \theta_{b}} F_{2}^{b}+\frac{1}{2} h_{b}^{2} A_{b}^{2} s_{2 \theta_{b}}^{2} F_{3}^{b}+2 h_{\tau} h_{b} m_{\tau} A_{b} s_{2 \theta_{b}} F_{4}^{b} \\
& +h_{\tau} h_{b} A_{\tau} A_{b} s_{2 \theta_{\tau}} s_{2 \theta_{b}} F_{5}+4 h_{\tau} h_{b} m_{\tau} m_{b} F_{6} \text {, } \\
& -\left(\Delta \mathcal{M}_{S}^{2}\right)_{12}^{\mathrm{eff}}=h_{\tau}^{2} \mu m_{\tau} s_{2 \theta_{\tau}} F_{2}^{\tau}+\frac{1}{2} h_{\tau}^{2} A_{\tau} \mu s_{2 \theta_{\tau}}^{2} F_{3}^{\tau}+h_{\tau} h_{b} m_{b} \mu s_{2 \theta_{\tau}} F_{4}^{\tau} \\
& +h_{b}^{2} \mu m_{b} s_{2 \theta_{b}} F_{2}^{b}+\frac{1}{2} h_{b}^{2} A_{b} \mu s_{2 \theta_{b}}^{2} F_{3}^{b}+h_{\tau} h_{b} m_{\tau} \mu s_{2 \theta_{b}} F_{4}^{b} \\
& +\frac{1}{2} h_{\tau} h_{b} s_{2 \theta_{\tau}} s_{2 \theta_{b}} \mu\left(A_{b}+A_{\tau}\right) F_{5} \\
& \left(\Delta \mathcal{M}_{S}^{2}\right)_{22}^{\mathrm{eff}}=\frac{1}{2} h_{\tau}^{2} \mu^{2} s_{2 \theta_{\tau}}^{2} F_{3}^{\tau}+\frac{1}{2} h_{b}^{2} \mu^{2} s_{2 \theta_{b}}^{2} F_{3}^{b}+h_{\tau} h_{b} \mu^{2} s_{2 \theta_{\tau}} s_{2 \theta_{b}} F_{5} \\
& v_{1}^{2} \Sigma_{1}=m_{\tau} A_{\tau} s_{2 \theta_{\tau}} F^{\tau}+2 m_{\tau}^{2} G^{\tau}+m_{b} A_{b} s_{2 \theta_{b}} F^{b}+2 m_{b}^{2} G^{b}, \\
& -v_{2}^{2} \Sigma_{2}=m_{\tau} \mu \tan \beta s_{2 \theta_{\tau}} F^{\tau}+m_{b} \mu \tan \beta s_{2 \theta_{b}} F^{b} \\
& \Delta m_{A}^{2}=-\frac{1}{c_{\beta} s_{\beta}}\left(2 h_{b} h_{\tau} F_{A}-\frac{h_{\tau}^{2} \mu A_{\tau}}{m_{\tilde{\tau}_{1}}^{2}-m_{\tilde{\tau}_{2}}^{2}} F^{\tau}-\frac{h_{b}^{2} \mu A_{b}}{m_{\tilde{b}_{1}}^{2}-m_{\tilde{b}_{2}}^{2}} F^{b}\right)
\end{aligned}
$$


In the equations above, $A_{\tau}$ and $A_{b}$ are the soft supersymmetry-breaking trilinear couplings of the Higgs fields to the stau and sbottom squarks, $\mu$ is the Higgs mass term 11 in the superpotential, $\tan \beta=v_{2} / v_{1}$ is the ratio of the two Higgs vacuum expectation values and $s_{2 \theta_{q}}(q=\tau, b)$ refer to the usual field-independent squark mixing angles. The functions $F_{i}^{q}(i=1,2,3,4), F_{5}, F_{6}, F^{q}, G^{q}$ and $F_{A}$ are combinations of the derivatives of $\Delta V_{b \tau}$ with respect to the field-dependent parameters, computed at the minimum of the effective potential; their explicit expressions are, in units of $N_{c} /\left(16 \pi^{2}\right)^{2}$ :

$$
\begin{aligned}
& F_{1}^{\tau}=-\frac{1}{2} h_{b} h_{\tau} s_{2 \theta_{b}} s_{2 \theta_{\tau}} I_{b} \frac{m_{\tilde{\tau}_{1}}^{2}-m_{\tilde{\tau}_{2}}^{2}}{m_{\tilde{\tau}_{1}}^{2} m_{\tilde{\tau}_{2}}^{2}} \\
& F_{2}^{\tau}=\frac{h_{b} h_{\tau} s_{2 \theta_{b}} I_{b}}{2 s_{2 \theta_{\tau}} m_{\tilde{\tau}_{1}}^{2} m_{\tilde{\tau}_{2}}^{2}\left(m_{\tilde{\tau}_{1}}^{2}-m_{\tilde{\tau}_{2}}^{2}\right)}\left[s_{2 \theta_{\tau}}^{2}\left(m_{\tilde{\tau}_{1}}^{4}-m_{\tilde{\tau}_{2}}^{4}\right)+2 c_{2 \theta_{\tau}}^{2} m_{\tilde{\tau}_{1}}^{2} m_{\tilde{\tau}_{2}}^{2} \ln \frac{m_{\tilde{\tau}_{1}}^{2}}{m_{\tilde{\tau}_{2}}^{2}}\right] \text {, } \\
& F_{3}^{\tau}=\frac{h_{b} h_{\tau} s_{2 \theta_{b}} I_{b}}{2 s_{2 \theta_{\tau}} m_{\tilde{\tau}_{1}}^{2} m_{\tilde{\tau}_{2}}^{2}\left(m_{\tilde{\tau}_{1}}^{2}-m_{\tilde{\tau}_{2}}^{2}\right)}\left[2\left(3 s_{2 \theta_{\tau}}^{2}-2\right) m_{\tilde{\tau}_{1}}^{2} m_{\tilde{\tau}_{2}}^{2} \frac{m_{\tilde{\tau}_{1}}^{2}+m_{\tilde{\tau}_{2}}^{2}}{m_{\tilde{\tau}_{1}}^{2}-m_{\tilde{\tau}_{2}}^{2}} \ln \frac{m_{\tilde{\tau}_{1}}^{2}}{m_{\tilde{\tau}_{2}}^{2}}\right. \\
& \left.-s_{2 \theta_{\tau}}^{2}\left(m_{\tilde{\tau}_{1}}^{2}+m_{\tilde{\tau}_{2}}^{2}\right)^{2}+8 c_{2 \theta_{\tau}}^{2} m_{\tilde{\tau}_{1}}^{2} m_{\tilde{\tau}_{2}}^{2}\right], \\
& F_{4}^{\tau}=\frac{h_{b} h_{\tau} s_{2 \theta_{b}}}{2 s_{2 \theta_{\tau}}\left(m_{\tilde{\tau}_{1}}^{2}-m_{\tilde{\tau}_{2}}^{2}\right)} \ln \frac{m_{\tilde{b}_{1}}^{2}}{m_{\tilde{b}_{2}}^{2}}\left[2 c_{2 \theta_{\tau}}^{2} I_{\tau}+s_{2 \theta_{\tau}}^{2}\left(m_{\tilde{\tau}_{1}}^{2}-m_{\tilde{\tau}_{2}}^{2}\right) \ln \frac{m_{\tilde{\tau}_{1}}^{2} m_{\tilde{\tau}_{2}}^{2}}{Q^{4}}\right], \\
& F_{5}=\frac{h_{b} h_{\tau}}{s_{2 \theta_{b}} s_{2 \theta_{\tau}}}\left[\frac{s_{2 \theta_{b}}^{2} c_{2 \theta_{\tau}}^{2} I_{\tau}}{m_{\tilde{\tau}_{1}}^{2}-m_{\tilde{\tau}_{2}}^{2}} \ln \frac{m_{\tilde{b}_{1}}^{2} m_{\tilde{b}_{2}}^{2}}{Q^{4}}+\frac{s_{2 \theta_{\tau}}^{2} c_{2 \theta_{b}}^{2} I_{b}}{m_{\tilde{b}_{1}}^{2}-m_{\tilde{b}_{2}}^{2}} \ln \frac{m_{\tilde{\tau}_{1}}^{2} m_{\tilde{\tau}_{2}}^{2}}{Q^{4}}\right. \\
& \left.-\frac{2 I_{\tau} I_{b}\left(1-c_{2 \theta_{b}}^{2} c_{2 \theta_{\tau}}^{2}\right)}{\left(m_{\tilde{b}_{1}}^{2}-m_{\tilde{b}_{2}}^{2}\right)\left(m_{\tilde{\tau}_{1}}^{2}-m_{\tilde{\tau}_{2}}^{2}\right)}+\frac{1}{2} s_{2 \theta_{b}}^{2} s_{2 \theta_{\tau}}^{2} \ln \frac{m_{\tilde{b}_{1}}^{2} m_{\tilde{b}_{2}}^{2}}{Q^{4}} \ln \frac{m_{\tilde{\tau}_{1}}^{2} m_{\tilde{\tau}_{2}}^{2}}{Q^{4}}\right], \\
& F_{6}=\frac{1}{2} h_{b} h_{\tau} s_{2 \theta_{b}} s_{2 \theta_{\tau}} \ln \frac{m_{\tilde{b}_{1}}^{2}}{m_{\tilde{b}_{2}}^{2}} \ln \frac{m_{\tilde{\tau}_{1}}^{2}}{m_{\tilde{\tau}_{2}}^{2}}, \\
& F^{\tau}=\frac{h_{b} h_{\tau} s_{2 \theta_{b}} I_{b}}{2 s_{2 \theta_{\tau}}\left(m_{\tilde{\tau}_{1}}^{2}-m_{\tilde{\tau}_{2}}^{2}\right)}\left[2 c_{2 \theta_{\tau}}^{2} I_{\tau}+s_{2 \theta_{\tau}}^{2}\left(m_{\tilde{\tau}_{1}}^{2}-m_{\tilde{\tau}_{2}}^{2}\right) \ln \frac{m_{\tilde{\tau}_{1}}^{2} m_{\tilde{\tau}_{2}}^{2}}{Q^{4}}\right] \text {, } \\
& G^{\tau}=\frac{1}{2} h_{b} h_{\tau} s_{2 \theta_{b}} s_{2 \theta_{\tau}} I_{b} \ln \frac{m_{\tilde{\tau}_{1}}^{2}}{m_{\tilde{\tau}_{2}}^{2}} \\
& F_{A}=\frac{2 h_{b} h_{\tau} m_{b} m_{\tau} \mu^{2}\left(A_{b}-A_{\tau}\right)^{2} \tan \beta I_{b} I_{\tau}}{s_{2 \theta_{b}} s_{2 \theta_{\tau}}\left(m_{\tilde{\tau}_{1}}^{2}-m_{\tilde{\tau}_{2}}^{2}\right)^{2}\left(m_{\tilde{b}_{1}}^{2}-m_{\tilde{b}_{2}}^{2}\right)^{2}} .
\end{aligned}
$$

The functions $F_{i}^{b}, F^{b}$ and $G^{b}$ can be obtained from their tau counterparts through the

\footnotetext{
${ }^{11}$ Note that we employ here the opposite convention for the sign of $\mu$ with respect to Ref. [26], which explains the minus signs appearing in eqs. (B4), (B7) and (B8).
} 
replacement $\tau \leftrightarrow b$. The function $I_{q}(q=\tau, b)$ entering the above equations is defined as:

$$
I_{q}=m_{\tilde{q}_{1}}^{2}\left(\ln \frac{m_{\tilde{q}_{1}}^{2}}{Q^{2}}-1\right)-m_{\tilde{q}_{2}}^{2}\left(\ln \frac{m_{\tilde{q}_{2}}^{2}}{Q^{2}}-1\right) .
$$

The results in Eqs. (Bי $)-(\mathrm{B} 17)$ are valid when the one-loop $\mathcal{O}\left(\alpha_{\tau}\right)$ and $\mathcal{O}\left(\alpha_{b}\right)$ corrections are written in terms of $\overline{\mathrm{DR}}$ parameters computed at the renormalisation scale $Q^{2}$. In alternative, an on-shell renormalisation scheme for the tau/stau and bottom/sbottom sectors that avoids the appearance of $\tan \beta$-enhanced terms in the two-loop part of the results should be devised along the lines of Refs. [24, 26].

\section{References}

[1] For a recent review and further references see S. P. Martin, hep-ph/9709356.

[2] A. Djouadi et al. [MSSM Working Group Collaboration], hep-ph/9901246.

[3] H. P. Nilles, Phys. Lett. B115 (1982) 193; Nucl. Phys. B217 (1983) 366;

A. H. Chamseddine, R. Arnowitt and P. Nath, Phys. Rev. Lett. 49 (1982) 970;

R. Barbieri, S. Ferrara and C. A. Savoy, Phys. Lett. B119 (1982) 343;

L. Hall, J. Lykken and S. Weinberg, Phys. Rev. D27 (1983) 2359;

S. K. Soni and H. A. Weldon, Phys. Lett. B126 (1983) 215;

N. Ohta, Prog. Theor. Phys. 70 (1983) 542.

[4] M. Dine and A. E. Nelson, Phys. Rev. D48 (1993) 1277 hep-ph/9303230;

M. Dine, A. E. Nelson and Y. Shirman, Phys. Rev. D51 (1995) 1362 hep-ph/9408384;

M. Dine, A. E. Nelson, Y. Nir and Y. Shirman, Phys. Rev. D53 (1996) 2658 hep-ph/9507378;

for a review see G. F. Giudice and R. Rattazzi, Phys. Rept. 322 (1999) 419 hep-ph/9801271.

[5] L. Randall and R. Sundrum, Nucl. Phys. B557 (1999) 79 hep-ph/9810155];

G. F. Giudice, M. A. Luty, H. Murayama and R. Rattazzi, JHEP 9812 (1998) 027 hep-ph/9810442;

J. A. Bagger, T. Moroi and E. Poppitz, JHEP 0004 (2000) 009 [hep-th/9911029].

[6] See for instance A. Brignole, L. E. Ibanez and C. Munoz, Nucl. Phys. B422 (1994) 125 hep-ph/9308271], Erratum ibid. B436 (1995) 747;

S. A. Abel, B. C. Allanach, F. Quevedo, L. Ibanez and M. Klein, JHEP 0012 (2000) 026 hep-ph/0005260;

P. Binetruy, M. K. Gaillard and B. D. Nelson, Nucl. Phys. B604 (2001) 32 
hep-ph/0011081];

B. C. Allanach, S. F. King and D. A. J. Rayner, hep-ph/0403255.

[7] H. Baer, F. E. Paige, S. D. Protopopescu and X. Tata, hep-ph/0001086.

[8] B. C. Allanach, Comput. Phys. Commun. 143 (2002) 305 hep-ph/0104145], available from http://allanach.home.cern.ch/allanach/softsusy.html.

[9] A. Djouadi, J. L. Kneur and G. Moultaka, hep-ph/0211331, available from http://w3.lpm.univ-montp2.fr/ ${ }^{\sim}$ kneur/Suspect.

[10] W. Porod, Comput. Phys. Commun. 153 (2003) 275 hep-ph/0301101], available from http://www-theorie.physik.unizh.ch/ porod/SPheno.html.

[11] B. C. Allanach, hep-ph/0110227;

B. C. Allanach, S. Kraml and W. Porod, hep-ph/0207314; JHEP 0303 (2003) 016 hep-ph/0302102;

A. Djouadi, hep-ph/0211357.

[12] For a recent review and further references see M. Carena and H. E. Haber, Prog. Part. Nucl. Phys. 50 (2003) 63 [hep-ph/0208209].

[13] Y. Okada, M. Yamaguchi and T. Yanagida, Prog. Theor. Phys. 85 (1991) 1; Phys. Lett. B262, (1991) 54;

J. R. Ellis, G. Ridolfi and F. Zwirner, Phys. Lett. B257 (1991) 83; Phys. Lett. B262 (1991) 477;

H. E. Haber and R. Hempfling, Phys. Rev. Lett. 66 (1991) 1815.

[14] P. H. Chankowski, S. Pokorski and J. Rosiek, Phys. Lett. B274 (1992) 191;

A. Brignole, Phys. Lett. B281 (1992) 284.

[15] A. Dabelstein, Z. Phys. C67 (1995) 495 hep-ph/9409375.

[16] D. M. Pierce, J. A. Bagger, K. T. Matchev and R. J. Zhang, Nucl. Phys. B491 (1997) 3 [hep-ph/9606211.

[17] M. Carena, J. R. Espinosa, M. Quiros and C. E. Wagner, Phys. Lett. B355 (1995) 209 hep-ph/9504316;

M. Carena, M. Quiros and C. E. Wagner, Nucl. Phys. B461 (1996) 407 hep-ph/9508343;

J. R. Espinosa and I. Navarro, Nucl. Phys. B615 (2001) 82 [hep-ph/0104047].

[18] H. E. Haber, R. Hempfling and A. H. Hoang, Z. Phys. C75 (1997) 539 hep-ph/9609331.

[19] R. Hempfling and A. H. Hoang, Phys. Lett. B331 (1994) 99 hep-ph/9401219. 
[20] S. Heinemeyer, W. Hollik and G. Weiglein, Phys. Rev. D58 (1998) 091701 hep-ph/9803277]; Phys. Lett. B440 (1998) 296 [hep-ph/9807423]; Eur. Phys. J. C9 (1999) 343 hep-ph/9812472]; Phys. Lett. B455 (1999) 179 [hep-ph/9903404.

[21] R. J. Zhang, Phys. Lett. B447 (1999) 89 hep-ph/9808299;

J. R. Espinosa and R. J. Zhang, JHEP 0003 (2000) 026 hep-ph/9912236.

[22] J. R. Espinosa and R. J. Zhang, Nucl. Phys. B586 (2000) 3 [hep-ph/0003246].

[23] G. Degrassi, P. Slavich and F. Zwirner, Nucl. Phys. B611 (2001) 403 hep-ph/0105096.

[24] A. Brignole, G. Degrassi, P. Slavich and F. Zwirner, Nucl. Phys. B643 (2002) 79 hep-ph/0206101.

[25] A. Brignole, G. Degrassi, P. Slavich and F. Zwirner, Nucl. Phys. B631 (2002) 195 hep-ph/0112177].

[26] A. Dedes, G. Degrassi and P. Slavich, Nucl. Phys. B672 (2003) 144 hep-ph/0305127.

[27] R. Arnowitt and P. Nath, Phys. Rev. D46 (1992) 3981;

V. D. Barger, M. S. Berger and P. Ohmann, Phys. Rev. D49 (1994) 4908 hep-ph/9311269].

[28] A. Dedes and P. Slavich, Nucl. Phys. B657 (2003) 333 hep-ph/0212132.

[29] S. P. Martin, Phys. Rev. D65 (2002) 116003 hep-ph/0111209; Phys. Rev. D66 (2002) 096001 hep-ph/0206136.

[30] S. P. Martin, Phys. Rev. D67 (2003) 095012 [hep-ph/0211366].

[31] S. Heinemeyer, W. Hollik and G. Weiglein, Comp. Phys. Commun. 124 (2000) 76 [hep-ph/9812320];

M. Frank, S. Heinemeyer, W. Hollik and G. Weiglein, hep-ph/0202166.

[32] S. Heinemeyer, Eur. Phys. Jour. C22 (2001) 521 hep-ph/0108059;

M. Frank, S. Heinemeyer, W. Hollik and G. Weiglein, hep-ph/0212037; in preparation.

[33] G. Degrassi, S. Heinemeyer, W. Hollik, P. Slavich and G. Weiglein, Eur. Phys. J. C28 (2003) 133 hep-ph/0212020.

[34] B. C. Allanach et al., Eur. Phys. J. C25 (2002) 113 hep-ph/0202233.

[35] The Tevatron Electroweak Working Group [D0 Collaboration], hep-ex/0404010.

[36] A. Djouadi, M. Drees and J. L. Kneur, JHEP 0108 (2001) 055 hep-ph/0107316. 
[37] A. Dedes, S. Heinemeyer, P. Teixeira-Dias and G. Weiglein, hep-ph/9912249;

S. Ambrosanio, A. Dedes, S. Heinemeyer, S. Su and G. Weiglein, Nucl. Phys. B624 (2002) 3 [hep-ph/0106255];

J. R. Ellis, S. Heinemeyer, K. A. Olive and G. Weiglein, JHEP 0301 (2003) 006 hep-ph/0211206.

[38] A. Dedes, S. Heinemeyer, S. Su and G. Weiglein, Nucl. Phys. B674 (2003) 271 hep-ph/0302174].

[39] W. H. Press, S. A. Teukolsky, W. T. Vettering and B. P. Flannery, Numerical Recipes in $C++$, Cambridge University Press 2002.

[40] P. Skands et al., JHEP 0407 (2004) 036 hep-ph/0311123.

[41] M. Carena, D. Garcia, U. Nierste and C. E. Wagner, Nucl. Phys. B577 (2000) 88 hep-ph/9912516];

[42] T. Banks, Nucl. Phys. B303 (1988) 172;

L. J. Hall, R. Rattazzi and U. Sarid, Phys. Rev. D50 (1994) 7048 [hep-ph/9306309];

R. Hempfling, Phys. Rev. D49 (1994) 6168;

M. Carena, M. Olechowski, S. Pokorski and C. E. Wagner, Nucl. Phys. B426 (1994) 269 hep-ph/9402253.

[43] S. P. Martin, Phys. Rev. D68 (2003) 075002 hep-ph/0307101]; Phys. Rev. D70 (2004) 016005 hep-ph/0312092].

[44] A. Djouadi, J. Kalinowski and M. Spira, Comput. Phys. Commun. 108 (1998) 56 hep-ph/9704448.

[45] M. Carena, S. Heinemeyer, C. E. M. Wagner and G. Weiglein, hep-ph/9912223; Eur. Phys. J. C26 (2003) 601 [hep-ph/0202167].

[46] K. Hagiwara et al. [Particle Data Group Collaboration], Phys. Rev. D66 (2002) 010001.

[47] M. Carena, H. E. Haber, S. Heinemeyer, W. Hollik, C. E. M. Wagner and G. Weiglein, Nucl. Phys. B580 (2000) 29 [hep-ph/0001002].

[48] S. Heinemeyer, talk given at the International Conference on Linear Colliders LCWS 04, Paris 19-23 April 2004, and private communications.

[49] J. A. Aguilar-Saavedra et al. [ECFA/DESY LC Physics Working Group Collaboration], hep-ph/0106315.

[50] S. Heinemeyer, S. Kraml, W. Porod and G. Weiglein, JHEP 0309 (2003) 075 hep-ph/0306181]. 
[51] A. Djouadi, J. Kalinowski and P.M. Zerwas, Z. Phys. C70 (1996) 435 hep-ph/9511342;

A. Djouadi, M. Spira and P.M. Zerwas, Z. Phys. C70 (1996) 427 [hep-ph/9511344];

A. Djouadi and P. Gambino, Phys. Rev. D51 (1995) 218 [hep-ph/9406431].

[52] S. Dimopoulos and G. F. Giudice, Phys. Lett. B357 (1995) 573 hep-ph/9507282].

[53] J. A. Casas, A. Lleyda and C. Munoz, Nucl. Phys. B471 (1996) 3 hep-ph/9507294.

[54] A. Dedes, A. B. Lahanas and K. Tamvakis, Phys. Rev. D53 (1996) 3793 hep-ph/9504239;

S. Ambrosanio, G. D. Kribs and S. P. Martin, Phys. Rev. D56 (1997) 1761 hep-ph/9703211;

S. Su, Nucl. Phys. B573 (2000) 87 hep-ph/9910481].

[55] R. Barate et al. [LEP collaborations], Phys. Lett. B565 (2003) 61 [hep-ex/0306033].

[56] S. A. Abel and B. C. Allanach, Phys. Lett. B431 (1998) 339 hep-ph/9803476];

M. Lanzagorta and G. G. Ross, Phys. Lett. B349 (1995) 319 hep-ph/9501394];

B. Pendleton and G. G. Ross, Phys. Lett. B98 (1981) 291.

[57] S. A. Abel and B. C. Allanach, JHEP 0007 (2000) 037 hep-ph/9909448;

J. A. Casas, J. R. Espinosa and H. E. Haber, Nucl. Phys. B526 (1998) 3 hep-ph/9801365.

[58] M. B. Einhorn and D. R. T. Jones, Nucl. Phys. B196 (1982) 475;

J. Ellis, D. V. Nanopoulos and S. Rudaz, Nucl. Phys. B202 (1982) 43;

V. D. Barger, M. S. Berger, P. Ohmann and R. J. N. Phillips, Phys. Lett. B314 (1993) 351 hep-ph/9304295];

M. Carena, S. Pokorski and C. E. M. Wagner, Nucl. Phys. B406, 59 (1993) hep-ph/9303202].

[59] See for instance S. Choi et al., Eur. Phys. J. C14 (2000) 535 [hep-ph/0002033 and Eur. Phys. J. C8 (1999) 669 hep-ph/9812236.

[60] A. Djouadi, J. Kalinowski and P.M. Zerwas, Mod. Phys. Lett. A7 (1992) 1765 and Z. Phys. C54 (1992) 255;

J. Dai, J. F. Gunion and R. Vega, Phys. Lett. B387 (1996) 801 hep-ph/9607379];

J. F. Gunion, T. Han, J. Jiang and A. Sopczak, Phys. Lett. B565 (2003) 42 hep-ph/0212151.

[61] S. Y. Choi, J. Kalinowski, J. S. Lee, M. M. Muhlleitner, M. Spira and P. M. Zerwas, hep-ph/0404119.

[62] A. Bartl, K. Hidaka, T. Kernreiter and W. Porod, Phys. Rev. D66 (2002) 115009 hep-ph/0207186. 
[63] S. Heinemeyer, W. Hollik and G. Weiglein, JHEP 0006 (2000) 009 [hep-ph/9909540].

[64] F. Jegerlehner, hep-ph/0312372.

[65] See for instance A. Djouadi, Phys. Lett. B435 (1998) 101 [hep-ph/9806315].

[66] CMS Collaboration, Technical Proposal, report CERN/LHCC/94-38 (1994); ATLAS Collaboration, Technical Design Report, CERN/LHCC/99-15 (1999);

Proceedings of the Les Houches Workshops "Physics at TeV colliders", A. Djouadi et al., hep-ph/0002258 (1999) and D. Cavalli et al., hep-ph/0203056 (2001).

[67] V. Barger et al., Phys. Rev. D49 (1994) 79 [hep-ph/9306270];

S.Y. Choi, Phys. Lett. B553 (2003) 61 [hep-ph/0210077].

[68] A. Djouadi et al., in preparation.

[69] S. P. Martin, hep-ph/0405022. 